\title{
Universal Aspects of Entanglement in Quantum Field theory and String Theory
}

\author{
Gabriel Wong
}

B.A., University of California, Berkeley 2003

A Dissertation presented to the Graduate Faculty

of the University of Virginia in Candidacy for the Degree of Doctor of Philosophy

Department of Physics

University of Virginia

July, 2017 
(c) Gabriel Wong 2017 All Rights Reserved 


\section{Acknowledgements}

"Only in the leap from the lion's head will he prove his worth..." So uttered Indiana Jones in the finale of "Indiana Jones and the Last Crusade" as he looked across an impassible chasm separating him from the final resting place of the holy grail. The riddle is a clue- to reach the grail, Indy must take a leap of faith, literally stepping out onto thin air to walk across the chasm. In the movie, Indy takes the leap, hand over his heart, and finds to his surprise a nearly invisible bridge under his feet that carries him across the abyss. A liberal, perhaps Kierkegaardian interpretation of this moment would contend that the bridge really didn't exist before Indy step foot on it. Rather, it was his leap of faith that created it. Likewise, the passage from student to researcher demands such a leap. In the absence of any religious conviction on my part, this leap was made possible by a long list of people who believed in me and supported me along the way.

This list begins with my advisers Diana Vaman and Israel Klich for their guidance, collaboration and for helping me chart my own course, Leopoldo Pando-Zayas for getting me started on my first project on entanglement and for encouraging me to search for the deep questions, William Donnelly for being a generous collaborator and teaching me about entanglement edge modes. Like all great human endeavors, Physics is a living discipline that is passed down from generation to generation, from

heart to heart. Among the physicists whose mentoring and encouragement helped shape my view of physics are Ori Ganor, Petr Horava, Robert Littlejohn, Bruno 
Zumino, Peter Arnold, Hank Thacker, Paul Fendley, Geoffrey Teo, Joan Simons, and Shinsei Ryu.

I have benefited from the friendship and knowledge of many graduate students and post-docs at various institutions. Anthony Tagliaferro, Chris Beem, Liang Yu, Hal Haggard and Badr Albanna at UC Berkeley, Yifei Shi, Abha Rajan, Phillip Szepietowski, Jean-Marie Stephan, and Zhao Zhang at University of Virginia, Tarun Grover, Louk Rademaker, Thomas Scaffidi, and Rina Takeshima at the KITP.

Two-thirds of this thesis was written while I was supported as a visiting graduate student at KITP Santa Barbara and the Perimeter Institute. I thank Joe Polchinski and Rob Myers for hosting me at these wonderful institutions, and the Bistro staff at Perimeter Institute for making each meal a delight. I owe a debt of gratitude to the University of Virginia for their support via a four-year presidential fellowship, which gave me a chance to re-boot my physics graduate career in the lovely town of Charlottesville.

Thank you to Sandra Bain Cushman, Jerry Sontag, Suzanne Berger, Ronit Corry and Laura Klein for teaching me the ways of the Alexander Technique, which has been indispensable in the upkeep of my physical and mental health.

I thank my parents for their un-ending support in all of my endeavors, my dear friends Melissa Murphy for her steady companionship and Ayon Roy for encouraging me to live by my convictions, and my brother Andrew for always being ready to lend a helping hand.

Finally, I would like to thank the musicians and music teachers who welcomed me into the their world: Marika Kuzma for introducing me to the magic of choral music, for giving me the opportunity to conduct her impeccably trained choruses and for getting me into Indiana University's choral conducting program, Betty Wu and Martha Wasley for wonderful lessons at the piano, David Milnes for generously opening his orchestral conducting class to me and giving me my first conducting experience 
on the podium, Jennifer Bailey for encouraging me to continue my conducting studies, Michael Slon for the many unforgettable concerts with the UVA choruses, Scott Seki, Rondy Lazro, and Jack Marshall for introducing me to the joys of barbershop singing, Mursel Yavuz for his friendship and musical inspiration, and Carmen Tellez for her constant encouragement during my brief stint as a choral conducting graduate student.

It may seem strange to close with a dedication to music and musicians in a Ph.d thesis about physics- Indeed the love of music was in part responsible for the long and circuitous route I took in completing this doctorate, an undertaking which essentially began more than a decade ago in UC Berkeley. But as Adorno once remarked:

"...This inadequacy resembles that of life, which describes a wavering, deviating line, disappointing by comparison with it's premisses, and yet which only in this actual course, always less than it should be, is able...to represent an un-regimented one. If a life fulfilled its vocation directly, it would miss it's mark" 


\section{Table of Contents}

Acknowledgements $\ldots \ldots \ldots \ldots \ldots \ldots$ ii

List of Figures $\ldots \ldots \ldots \ldots \ldots \ldots \ldots \ldots \ldots \ldots \ldots \ldots$ vii

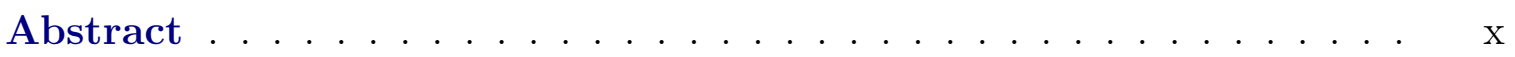

\section{Chapter}

I. Introduction $\ldots \ldots \ldots \ldots \ldots \ldots \ldots$

1.1 Introduction to quantum entanglement . . . . . . . . . . 1

1.2 Extended Hilbert space and edge modes . . . . . . . . . 5

1.3 Conical entropy and entanglement branes in string theory . . 7

II. Entanglement temperature and entanglement entropy of excited states . . . . . . . . . . . . . . . . . . 13

2.1 Introduction and summary . . . . . . . . . . . . . . . 13

2.2 Path Integral Derivation of the modular Hamiltonian . . . . 17

2.3 Examples of local modular Hamiltonians . . . . . . . . . . 20

2.3.1 modular Hamiltonians in 2D . . . . . . . . . 20

2.3.2 Modular Hamiltonians in higher dimensions . . . . . 24

2.4 CFT derivation of Entanglement Entropy for excited states . 26

2.5 A generalized first law for entanglement entropy . . . . . . 28

2.6 Holographic derivation and discussion of related papers . . . 30

2.6.1 Dynamical equations for entanglement entropy and entanglement density . . . . . . . . . . . 33

2.7 Conclusion . . . . . . . . . . . . . . . . . 36

2.8 Appendix A:Evaluating the ground state entanglement entropy from the modular Hamiltonian . . . . . . . . . . . . 38

2.9 Appendix B: Non-uniform excitation of 2D free scalar field . . 40

III. Entanglement branes in a two dimensional string theory . . . 43 
3.1 Introduction . . . . . . . . . . . . . . . . 43

3.2 Two-dimensional Yang-Mills theory as a closed string theory . 49

3.2.1 The closed string Hilbert space . . . . . . . . . . . . 50

3.2 .2 Torus partition function . . . . . . . . . . . . 55

3.2 .3 Sphere partition function . . . . . . . . . . . 58

3.3 Angular quantization on the sphere and open strings . . . . . 62

3.3.1 Entanglement of the Hartle-Hawking state . . . . . 62

3.3.2 The open string Hilbert space . . . . . . . . . . . 67

3.3 .3 Electric fields and quadratic Casimir . . . . . . . . . 70

3.3.4 The open string partition function . . . . . . . . . 72

3.3 .5 The zero area limit . . . . . . . . . . . . . 75

3.3.6 The free chiral string . . . . . . . . . . . . . . 77

3.4 The coupled theory . . . . . . . . . . . . . . . . . . 79

3.4.1 The coupled closed string Hilbert space . . . . . . 79

3.4.2 Entanglement tubes and the E-brane boundary state 82

3.4.3 Open string description of the entanglement tubes . 85

3.4.4 Entanglement and modular Hamiltonian ...... 91

3.4.5 The non-chiral free string . . . . . . . . . . . . 93

3.5 Discussion and Future work . . . . . . . . . . . . . . 94

IV. Entanglement edge modes in Chern Simons theory . . . . . 99

4.1 Entanglement edge modes and topological phases . . . . . . . 99

4.2 Edge modes from the Euclidean Path integral . . . . . . . 101

4.2.1 Path integral definition of the reduced density matrix 101

4.2.2 The extended Hilbert space of Chern Simons theory 102

4.3 The entangling product for Chern Simons theory . . . . . . . 107

4.3 .1 Hilbert space on a disk . . . . . . . . . . . . . 107

4.3 .2 Gluing of Hilbert spaces _. . . . . . . . . . . . 111

4.4 Entanglement entropy . . . . . . . . . . . . . . . . . . 113

4.5 Conclusion . . . . . . . . . . . . . . . . . . . . . 114 


\section{List of Figures}

\section{Figure}

1.1 Particle interactions occur at Lorentz invariant points on the worldline where the particle splits and joins as in the left figure. Integration over these interaction points leads to the UV divergences of perturbative field theory. In string theory, interaction are described by the "pair of pants" diagram. The red and black dashed lines represent constant time slices in two Lorentz frames, and the black and red dots label the moment of splitting in the two frames. Unlike the particle case, there is no Lorentz invariant interaction point [43]. . . . . . .

1.2 In the path integral description, the partial trace over region $B$ is obtained by glueing together the figure on the left along region $B$, leaving a cut on region $A . \ldots \ldots \ldots$

1.3 In string theory, $\log Z(\beta)$ is a sum of connected string diagrams embedded in a Euclidean spacetime with a conical defect of angle $\beta$ at the entangling surface. Only closed string diagrams that intersect or encircle the entangling surface, such as those depicted above, contribute to the entanglement entropy. . . . . . . . . . . . . . . . 11

2.1 Evaluating $\rho_{A}$ along Euclidean time s . . . . . . . . . 14

2.2 Foliation of the Euclidean plane corresponding to angular quantization 21

2.3 A rotation on the $\mathrm{z}$ plane (represented as a Riemann sphere) is mapped to a conformal rotation on the $w$ plane . . . . . . . . . . .

3.1 The diagrams that dominate in the calculation of entanglement entropy are sphere diagrams such as the one depicted on the left. Sliced transverse to the entangling surface, as in the middle figure, this diagram describes a closed string emitted from a point on the entangling surface and then reabsorbed. Sliced in angular time $\phi$, the sphere diagram is a one loop open string diagram with the endpoints fixed on the entangling surface as depicted on the right. . . . . . . . . .

3.2 In string theory, $\log Z(\beta)$ is a sum of connected string diagrams embedded in a Euclidean spacetime with a conical defect of angle $\beta$ at the entangling surface. Only closed string diagrams that intersect or encircle the entangling surface, such as those depicted above, contribute to the entanglement entropy. . . . . . . . . . . . . 
3.3 The closed string configuration corresponding to the permutation $\sigma: 1\rangle 2,2\rangle 3,3\rangle 1,4\rangle 5,5\rangle 4$. The cycle lengths $(3,2)$ correspond to the winding numbers of the closed strings. . . . . . . . . . . . . . .

3.4 A 3-sheeted covering map of the torus $T^{2}$ with two interaction branch points $q_{1}, q_{2}$. The covering map $\nu$ is defined here by vertical projection. A counterclockwise loop encircling the target space branch point $q_{1}$ lifts to a permutation $p_{1}: 1 \rightarrow 1,2 \rightarrow 3,3 \rightarrow 2$. A vertical time slicing of $\Sigma$ shows an initial closed string breaking into two and then joining back together again. . . . . . . . . . . . . . . .

3.5 $Z_{S^{2}}^{+}$can be expressed as a sum over worldsheets with holes corresponding to closed strings that wind around the $\Omega$-points on $S^{2}$. . .

3.6 By closing up the boundaries of the worldsheet into branch points and introducing appropriate branch cuts, we can present the worldsheet as a covering space of the sphere, with covering map represented by vertical projection. The first is a single covering with no interaction and $\Omega$-point singularities. The second is a double cover of the sphere with two $\Omega$-points. The third term corresponds to the "pair of pants" diagram, now presented as a double cover with an interaction branch point inserted, which is connected to an $\Omega$-point via a branch cut. .

3.7 The figure on the left shows a term in the Gross-Taylor expansion corresponding to a two-sheeted branched covering of the sphere with two $\Omega$-point singularities. These two fixed points on the target sphere are labelled by the permutation $\sigma: 1 \rightarrow 2,2 \rightarrow 1$, since circling these points interchanges the two sheets. On the right, we have opened up each branch point on the covering space into a single connected boundary, representing an external closed string state given by $|\sigma\rangle$. In the open string channel, this is interpreted as a finite temperature loop diagram in which an open string anchored on the $\Omega$-points winds twice around the sphere. . . . . . . . . . . . . . . .

3.8 The Yang-Mills path integral on a hemisphere gives the unnormalized Hartle-Hawking wave function, and the path integral on the sphere computes the square of its norm. Changing the periodicity of the angular coordinate $\phi$ to $2 \pi n$ yields $\operatorname{tr}\left(\rho_{V}^{n}\right)$. . . . . . . . . . . .

3.9 The state $|I J\rangle$ represents a configuration of $n$ open strings with ChanPaton indices $\left(i_{1}, j_{1}\right) \ldots\left(i_{n}, j_{n}\right) \ldots \ldots \ldots$

Evolution under the open string modular Hamiltonian is represented as cylindrical worldsheets where time runs transverse to branch cuts. The figure shows a pair of open strings and their Chan-Paton indices at two different time slices. The indices on the left endpoints are exchanged as they pass through the branch cut. (The branch cut on the "back side" the cylinder connects both boundaries and does not correspond to open string interaction. This is the same branch cut that appears in figure 3.7.) . . . . . . . . . . . . . . . 
3.11 In the free theory, the relevant diagrams are cylinders that wind around the direction corresponding to the entanglement time. The open ends of the cylinder are wrapped around the two $\Omega$-points. . .

3.12 Shown are two diagrams appearing in $\lim _{A\rangle 0}\left\langle\Omega\left|e^{-\frac{\lambda A}{2 N} C_{2}}\right| \Omega\right\rangle$. On the left,we have a decoupled closed string and anti string configuration. In the physical Hilbert space, strings and anti strings are entangled, leading to the diagram on the right where tubes $\nu$ and $\nu^{\prime}$ connect string-anti string pairs. . . . . . . . . . . . . .

3.13 The left figure illustrates the diagrammatic computation for the number of open string edge modes for $n=\bar{n}=1$. As expected, this gives the square of the dimension of the adjoint representation, $\left(N^{2}-1\right)^{2}$.

3.14 On the left we have a diagram representing the states $U_{I J} U_{K L}^{\dagger}$. The diagram in the middle represents constraints imposed on $U_{I J} U_{K L}^{\dagger}$ obtained by contracting a pair of right endpoints. The right most figure shows another diagram with contractions on both the left and right endpoints . . . . . . . . . . . . . . . . .

3.15 The relevant connected diagrams for the free non-chiral string are cylinders that wind around entanglement time circle, and which collapse into an $\Omega$-point multiple times, each time re-emerging with the opposite orientation. . . . . . . . . . . . . . . . . . .

4.1 The wavefunctional prepared by the Euclidean path integral can be sliced in angular time $\theta$, provided we remove an $\epsilon$ neighborhood of the entangling surface. When viewed as the initial and final time slices in $\theta$, region $A$ and $B$ are assigned opposite orientations relative to the ambient space $A \cup B$. Thus they support the Hilbert space of a chiral and anti-chiral edge CFT, which transforms non-trivially under the boundary gauge group. In addition to parity, switching from $t$ to $\theta$ also involves a time reversal because they have opposite orientations on region $B$ at the $t=0$ time slice. Sliced in angular time, the Euclidean path integral prepares a Thermofield double state in which the left right entanglement of these CFT's leads to singlet state under a diagonal action of the boundary gauge group on $\partial A$ and $\partial B \ldots \ldots \ldots \ldots \ldots \ldots$ 


\begin{abstract}
Universal Aspects of Entanglement in Quantum Field Theory and String Theory by Gabriel Wong
\end{abstract}

Advisor: Israel Klich and Diana Vaman

We apply path integral methods to derive universal aspects of quantum entanglement in quantum field theory and string theory. These methods were originally used to understand vacuum entanglement between half spaces in Lorentz-invariant quantum field theories. In this work we begin by generalizing these results to spherical subregion in conformally invariant field theories and deriving a first law for entanglement entropy. For holographic CFT's, we also provide a simple derivation of the bulk entanglement first law. Next we address the question of entanglement and Hilbert space factorization in string theory by studying a simple two dimensional string theory dual to two-dimensional Yang Mills. Even though this is a closed string theory, we find that open strings appear upon restriction to a spatial subregion, as first noted by Susskind and Uglum [80]. We show that the entangling surface acts as an "entanglement brane" on which open strings end, and host open string edge modes that are responsible for the entanglement entropy in the Hartle-Hawking vacuum. Finally, we elaborate on the extended Hilbert space factorization of Chern Simons theory and show how this arises naturally from a proper regularization of the entangling surface in the Euclidean path integral. The regularization amounts to stretching the entan- 
gling surface into a co-dimension one surface which hosts edge modes of the Chern Simons theory when quantized on a spatial subregion. 


\section{Chapter I}

\section{Introduction}

\subsection{Introduction to quantum entanglement}

In recent years, quantum information theory has found many important applications in high energy and condensed matter physics. The central idea that connects these different fields is quantum entanglement, which refers to a subtle correlation between different sub-systems that cannot be described by classical probabilities. Due to this correlation, an observer restricted to a sub-region of space sees a mixed state, even when the global state is pure. Phrased differently, this means that exact knowledge of the quantum state of the total system, does not imply exact knowledge about it's subsystems.

To define the notion of subregion $A$ in quantum mechanics, we must first express the Hilbert space $H$ as a tensor product:

$$
H=H_{A} \otimes H_{B}
$$

where $B$ is the compliment of region $A$. The factorization allows us to define the reduced density matrix $\rho_{A}$ from a state $\rho$ by tracing over the Hilbert space on $B$

$$
\rho_{A}=\operatorname{tr}_{B} \rho
$$


Physics inside the subregion $A$ is captured by the reduced density matrix because it reproduces expectation values of operators $O_{A}{ }^{1}$ supported on $A$ :

$$
\operatorname{Tr}\left(\rho O_{A}\right)=\operatorname{Tr}_{A}\left(\rho_{A} O_{A}\right)
$$

The pure state $\rho$ is said to be entangled if $\rho_{A}$ is mixed - the standard example is the spin singlet state of two spin $1 / 2$ particles:

$$
\begin{aligned}
|\psi\rangle & =\frac{1}{\sqrt{2}}\left(|0\rangle_{A}|1\rangle_{B}-|1\rangle_{A}|0\rangle_{B}\right) \\
\rho_{A} & =\frac{1}{2} \mathbf{1}_{2 \times 2}
\end{aligned}
$$

Due to quantum entanglement an observer in $A$ suffers information loss pertaining to region $A$ when he/she is restricted from accessing region degrees of freedom in $B$. This information loss can be quantified by the entanglement entropy:

$$
S_{A}=-\operatorname{Tr}_{A}\left(\rho_{A} \ln \rho_{A}\right)
$$

In a continuum theory, the Hilbert space factorization (1) requires a UV regularization of the co-dimension 2 entangling surface that separates region $A$ and $B$. Among other things, this is needed to define the partial trace (2) in the continuum. As we will show chapter 3 and 4 of this work, this regularization is more than a mere technicality: it introduces important edge physics into the study of entanglement between spatial regions.

The study of entanglement entropy was originally motivated by attempts to interpret black hole entropy as information loss by an observer restricted to the outside of the event horizon [11]. More recently, a new paradigm has emerged via work in AdS/CFT [88][83][36] which suggests that quantum entanglement is responsible for

\footnotetext{
${ }^{1}$ To be precise we should really write $O_{A} \otimes \mathbf{1}_{B}$
} 
the emergence of spacetime. The central relation that led to this far-reaching conjecture is the Ryu-Takayanagi (RT) formula [73], which is a generalization of the Bekenstein-Hawking formula relating the horizon area to black hole entropy. It applies to holographic quantum field theories that are dual to gravitational theories in one higher dimension. Such a holographic duality is powerful because it relates the classical physics of a bulk spacetime to a strongly coupled and therefore quantum mechanical regime of a gauge theory living at the asymptotic boundary of the spacetime. Explicitly, the RT formula states that, in the large N limit of the gauge theory, the entanglement entropy of a subregion $A$ in the boundary is given by the area of an extremal surface $\gamma_{A}$ anchored on the entangling surface $\partial A$ and extended into the bulk space time

$$
S_{A}=\frac{\operatorname{Area}\left(\gamma_{A}\right)}{4 G}
$$

Here $G$ is the Newton's constant. While the holographic theories satisfying (6) was originally derived in string theory, recent work suggest the RT formula is applicable in a much wider context, including many-body quantum states that are simulated by tensor networks.

While entanglement entropy provides an important measure of entanglement, the reduced density matrix $\rho_{A}$ is a more fundamental object. Since any $\rho_{A}$ is Hermitian and positive semidefinite, it may be expressed as

$$
\rho_{A}=\frac{e^{-H_{A}}}{Z_{A}}
$$

for some Hermitean operator $H_{A}$ and normalization factor $Z_{A}$. The Modular Hamiltonian $^{2} H_{A}$ is an effective hamiltonian inside region $A$. Unfortunately, $H_{A}$ is in general a highly nonlocal operator and does not offer an intuitive understanding of the physics inside $A$. However, in the presence of certain spacetime symmetries and for certain

\footnotetext{
${ }^{2}$ This is referred to as the entanglement Hamiltonian in the condensed matter literature
} 
choices of region $A, H_{A}$ can be expressed as an integral of a local density. The prime example for such a situation arises in the half space bipartition of the Minkowski vacuum, which underlies the phenomenon of Hawking and Unruh radiation. According to the Bisognano-Wichmann theorem [9, 10], the modular Hamiltonian of a half space in the Minkowski vacuum is the generator of Lorentz boost. This result was derived in the algebraic formulation of QFT, which makes use of the Kubo-Martin-Schwinger condition to establish $H_{A}$ as the generator of Lorentz boost. Recently this result was extended by Casini, Huerta and Myers [17] in the algebraic formalism. For a spherical region $A$ in a $\mathrm{CFT}$, they find that the modular Hamiltonian may be written explicitly in terms of the physical energy density $T_{00}$ :

$$
H_{A}=\int_{A} \beta(x) T_{00}(x)
$$

where $\beta(x)$ is a function we refer to as the inverse "entanglement temperature". In chapter 2, we will offer a simple path integral derivation of (8) and apply it to derive the entanglement entropy of states which are perturbatively close to the vacuum. This will lead to a universal relation between the change in entanglement entropy and the "modular" energy inside a spherical region $A$, commonly referred to as the entanglement first law:

$$
\delta S_{A}=\delta\left\langle H_{A}\right\rangle
$$

The right hand side of this equation is the change in the expectation value in the vacuum modular Hamiltonian, due to a small perturbation $\delta \rho$ to the vacuum state $\rho$. Equation (9) was first derived in the context of holographic field theories: our result generalizes the first law to general CFT's. 


\subsection{Extended Hilbert space and edge modes}

As noted earlier, in the continuum the factorization (1) requires a UV regularization that separates region $A$ and $B$. In principle such a regularization is provided by a lattice cutoff. However, in a gauge theory, the Gauss law constraint poses an obstruction to the naive factorization even on the lattice. Classically, a state is given by initial data specified along a Cauchy surface $\Sigma$, and the Gauss law constraint says that for physical states the initial data cannot be specified independently when we partition the system into two pieces. Similarly in quantum mechanics, the Gauss law constraint imposes a correlation between different spatial subsystems, so that even on a lattice there is no way to factorize the Hilbert space into two independent parts.

One way to resolve this problem is to relax Gauss's law so that it can be violated at the boundary of $A$ and $B$. Formally this requires embedding $H$ into an extended Hilbert Space [26]

$$
H \subset H_{A} \otimes H_{B}
$$

where $H_{A}$ and $H_{B}$ are allowed to contain surface charges at their respective boundaries that transform non-trivially under the boundary gauge group. These additional degrees of freedom, which we refer to as "entanglement edge modes", represent the minimal extension needed to construct a factorizable Hilbert space from $H$. The physical Hilbert space is contained inside a gauge invariant subspace of $H_{A} \otimes H_{B}$ : within the enlarged Hilbert space, one can trace over $B$ and define the entanglement entropy as in (5). In the physical states, the matching of the electric fields across the boundary leads to entanglement between the edge modes which contributes to total entanglement entropy. Explicitly, in a lattice gauge theory where each link supports a Hilbert space spanned by the representation matrix elements of the gauge group, 
the entanglement entropy takes the general form [25].

$$
S_{A}=-\sum_{R_{\partial A}} P\left(R_{\partial A}\right) \log P\left(R_{\partial A}\right)+\sum P\left(R_{\partial A}\right) \sum_{e \in \partial A} \log \operatorname{dim} R_{e}+\text { bulk EE }
$$

where $R_{\partial A}$ denotes the set of representations labeling the links on the boundary, $e$ denotes the individual edges on the boundary and $\operatorname{dim} R_{e}$ is the dimension of the representation $R_{e}$ associated with an edge $e$. The first two term come from the edge modes:the first is the Shannon entropy associated with the probability distribution $P\left(R_{\partial A}\right)$ of representations over the boundary, and the second is a term extensive in the size of the boundary that appears in non-abelian gauge theories. It may seem strange to include the entropy due to edge modes, which are gauge variant degrees of freedom added for the sake of factorizing the Hilbert space. However, recent work have shown that these terms have a real significance. For example in $U(1)$ Maxwell theory, the Shannon-entropy of the edge modes is necessary to reconcile the replica trick and thermal calculation of entanglement entropy [30, 31], and in topological gauge theories like Chern Simons theory, the $\log \operatorname{dim} R$ term is responsible for the topological entanglement entropy, which is an important diagnostic for topological phases. In Chapter 4, we will elaborate on the extended Hilbert space for Chern Simons theory, and show how the edge mode entanglement naturally arises from the appropriately regulated Euclidean path integral. The factorized state is a regularized Ishibashi state and reproduces the well known topological entanglement entropies. We illustrate how the same factorization arises from the gluing of two spatial subregions via the entangling product defined by Donnelly and Freidel [28]. 


\subsection{Conical entropy and entanglement branes in string the-} ory

Perhaps the most profound insight about gravity is its holographic nature. The Bekenstein-Hawking entropy formula

$$
S_{\mathrm{BH}}=\frac{A}{4 l_{\text {planck }}^{2}},
$$

(in units with $c=\hbar=1$ ) is an indication that the relevant degrees of freedom for describing the interior of a black hole — and possibly any region of space — reside on its boundary at Planckian density. The area scaling of the entropy could be naturally explained if the relevant entropy is the entanglement entropy of quantum fields in their vacuum state $[76,12,77]$. This is because the entanglement entropy of quantum fields across the black hole horizon also scales with the area, but with the ultraviolet cutoff playing the role of the Planck length. Thus in order to obtain a finite entropy consistent with (12), we have to incorporate gravity into the entanglement calculation, necessitating a theory of quantum gravity. Finiteness of the entropy is crucial to the resolution of the information paradox, as otherwise a black hole could in principle store an infinite amount of information at its stretched horizon ${ }^{3}$.

A leading candidate for such a theory of quantum gravity is string theory. String theory is perturbatively defined by generalizing the first quantized description of particle field theory. The world-line Feynman diagrams describing the spacetime history of particles are replaced by worldsheet diagrams, which are Riemann surfaces

\footnotetext{
3Imagine sending "information carrying" photons into a black hole at the same rate as that of the emitted Hawking radiation, so the black hole remains at constant mass. More precisely, imagine that each photon we send is entangled with a partner photon which we keep in a register outside the black hole, so that each photon sent in carries information about the entangled pair. If the entanglement entropy across the horizon is unbounded, then the entanglement between the ingoing and register photons can become arbitrarily large. This would imply the information carried by the ingoing photons becomes infinite, whereas the information stored by the black hole is finite and given by the Bekenstein Hawking formula. Assuming no information is carried by the outgoing radiation, this mismatch leads to an apparent information loss [78, 80].
} 

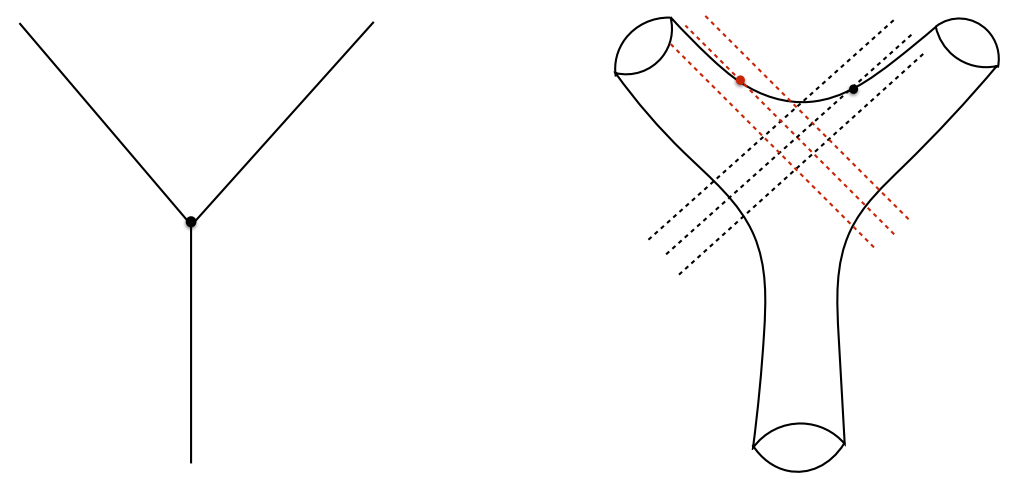

Figure 1.1:

Particle interactions occur at Lorentz invariant points on the worldline where the particle splits and joins as in the left figure. Integration over these interaction points leads to the UV divergences of perturbative field theory. In string theory, interaction are described by the "pair of pants" diagram. The red and black dashed lines represent constant time slices in two Lorentz frames, and the black and red dots label the moment of splitting in the two frames. Unlike the particle case, there is no Lorentz invariant interaction point [43].

swept out by the time evolution of a string. For the purpose of this thesis, it suffices to define the worldsheet theory via the Nambu-Goto action, which computes the area of the world sheet according to the target space metric G.

$$
I=\frac{1}{l_{\text {string }}^{2}} \int_{\text {worldsheet }} \sqrt{G}
$$

The basic interaction in string theory arises via the "pair of pants" diagram. Whereas particles split and join via vertices that can be localized to a spacetime point, there is no Lorentz invariant way to identify an interaction point for a string. This is a heuristic argument that suggest that string theory is free of the UV divergences of field theory, which arise due to the integration of the spacetime positions of the particle interactions. This led to the proposal $[78,80]$ that string theory provides a $U V$-finite entanglement entropy consistent with the Bekenstein Hawking formula.

More recently, the Ryu-Takayanagi formula (6) has provided another impetus for studying entanglement in string theory. As written, equation (6) represents the 
leading term in the $1 / N$ expansion of the entanglement entropy of the boundary gauge theory. The $1 / N$ corrections to (6) has been shown to be given by the entanglement entropy of bulk fields across the surface $\gamma_{A}$ [37]. Since the UV completion of the bulk theory is a string theory, it is natural to expect the entanglement of strings across $\gamma_{A}$ to be directly related to the microscopic origin of (6) in the bulk.

How do we study spatial entanglement in string theory? Intuitively, the nonlocal nature of strings would lead to the same kind of obstruction to Hilbert space factorization that we encounter in gauge theory; Formulated in terms in terms of Wilson loop variables, the gauge theory entanglement edge modes can be interpreted as charges appearing due to the cutting of the Wilson loop into Wilson lines ending on the entangling surface. It is tempting to generalize this property of gauge theory strings to the fundamental strings describing quantum gravity, which implies that the entangling surface acts as a brane on which open strings end. Unfortunately, unlike the gauge theory case, the second quantized Hilbert space of string theory is not well understood, making a direct application of the extended Hilbert space formalism difficult. Nevertheless, there is a way to probe the entanglement properties of fundamental strings using the replica trick, which we now review.

The replica trick is a convenient way to compute the logarithm in (5) via the moments $\operatorname{tr}\left(\tilde{\rho}_{A}^{n}\right)$ of the un normalized reduced density matrix, from which we obtain the entanglement entropy via

$$
S_{A}=\left.\frac{\partial}{\partial n} \log \operatorname{tr}\left(\tilde{\rho}_{A}^{n}\right)\right|_{n=1}
$$

This method is particular useful when we can prepare the quantum state by the Euclidean path integral. For such a state the un-normalized wave functional $\Psi[\phi(x), t=$ 0] of the field configuration $\phi(x)$ is given by the Euclidean path integral from time $t=0$ to $t=-\infty$, with boundary conditions $\phi(x)$ at the $t=0$ slice. As shown in figure 


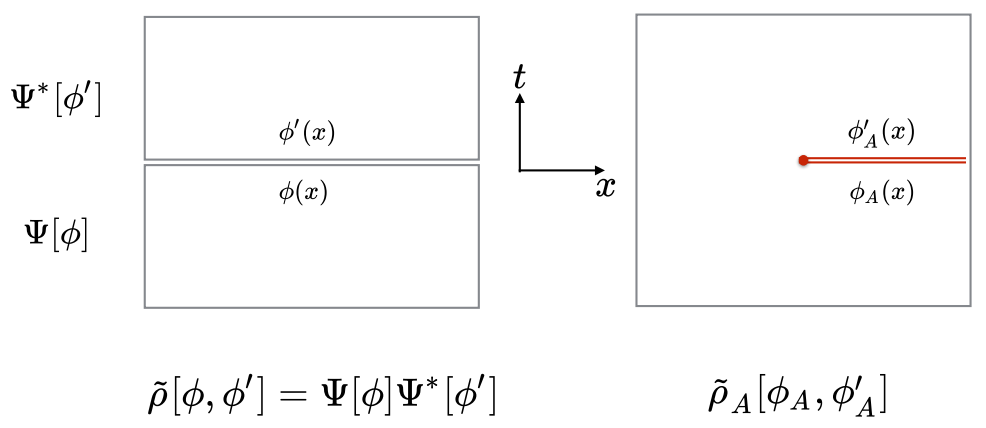

Figure 1.2: In the path integral description, the partial trace over region $B$ is obtained by glueing together the figure on the left along region $B$, leaving a cut on region $A$.

(1.2), the reduced density matrix elements $\tilde{\rho}_{A}\left(\phi_{A}, \phi_{A}^{\prime}\right)$ can then be represented as a path integral on the full space time with a cut along region $A$ that takes the boundary conditions $\phi_{A}, \phi_{A}^{\prime}$ on its upper and lower edge. For integer $n$, we can interpret $\operatorname{tr}\left(\tilde{\rho}_{A}^{n}\right)$ as the path integral on an $n$-sheeted spacetime, branched over the entangling surface. In this background, the entangling surface supports a conical singularity with excess angle of $2 \pi n$, and the replica trick (14) requires us to analytically continue $n$ near $n=1$. Setting $\beta=2 \pi n$ and interpreting the angular coordinate around the entangling surface as a thermal circle, we can re-interpret the moments $\operatorname{tr}\left(\tilde{\rho}_{A}^{n}\right)$ as an effective thermal partition function $Z(\beta)$. The entanglement entropy can then be identified with the thermal entropy of $Z(\beta)$

$$
S_{A}=\left.\left(1-\beta \partial_{\beta}\right) \log Z\right|_{\beta=2 \pi}
$$

The formula (15) represents the response of a partition function to the insertion of a small conical excess, and is referred to as the conical entropy. Note that it makes no explicit reference to a density matrix or a Hilbert space factorization.

Early work on entanglement in closed string theory [78, 80, 21, 20], focused on perturbative computations of the conical entropy (15), assuming that this is equal to the entanglement entropy of the string theory. In particular, Susskind and Uglum [? 


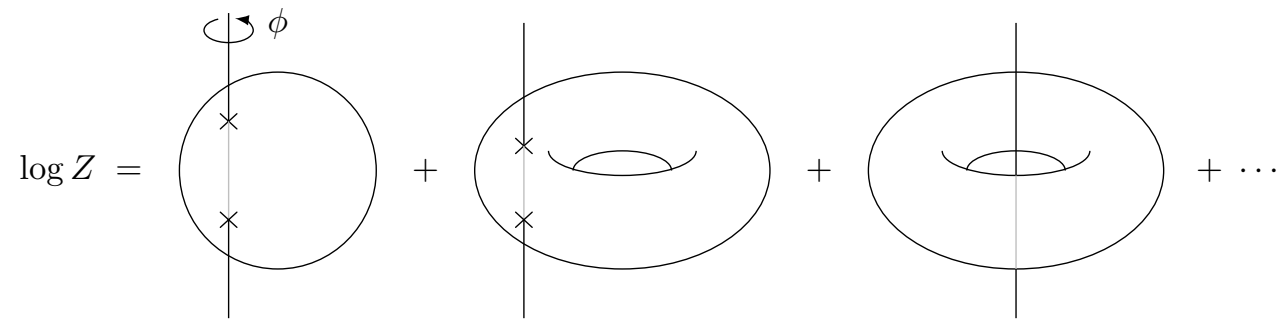

Figure 1.3:

In string theory, $\log Z(\beta)$ is a sum of connected string diagrams embedded in a Euclidean spacetime with a conical defect of angle $\beta$ at the entangling surface. Only closed string diagrams that intersect or encircle the entangling surface, such as those depicted above, contribute to the entanglement entropy.

] noted that the conical entropy receives a contribution only from diagrams where the closed string encircle or intersect the conical singularity, because only these diagrams have a non-linear dependence on $\beta$ due to the Nambu-Goto action. The relevant diagram giving rise to Bekenstein Hawking entropy is the classical sphere diagram intersecting the conical singularity as shown in figure 1.3. This "contact" term also exists in gauge theory, and was first computed by Kabat in $U(1)$ Maxwell theory. Surprisingly, the Maxwell contact term gives a dominant, negative contribution to the conical entropy in low spacetime dimensions. This led to doubts about whether the conical entropy can be given an entanglement interpretation, since the entanglement entropy is manifestly positive. Recently, this puzzle was resolved by Donnelly and Wall [? ] who showed that, properly regulated, the contact term is positive and arises from the entanglement of entropy of edge modes. The natural generalization to string theory suggests that the sphere diagram also calculates the edge mode entanglement entropy of string theory. Indeed Susskind and Uglum noted that when sliced in the angular time variable around the conical singularity, the sphere diagram describes the evolution of open strings anchored on the entangling surface. In terms of open strings, this is a one-loop diagram describing a trace over a single string Hilbert space:

$$
Z_{\text {sphere }}(\beta)=\operatorname{tr}_{\text {open strings }}\left(e^{-H_{\text {open }}}\right)
$$


Here $H_{\text {open }}$ is the open string modular hamtilonian that evolves the string around the entangling surface. This gives an explicit state-counting interpretation of the contact term and therefore the Bekenstein Hawking entropy! Just like the case of the gauge theory open string, this open string is really a closed string that is partially hidden behind the entangling surface. The open string states should therefore be interpreted as entanglement edge modes of the closed string theory, which are given a spacetime description as an entanglement brane.

Despite the elegance of Susskind and Uglum's argument, so far there has been no explicit realization of the open string edge modes. In chapter 3, we offer the first explicit calculation of these edge modes in the closed string theory dual to twodimensional Yang Mills. We will give a complete open string expansion of $Z(\beta)$ containing an explicit sum over edge modes, which we identify as the Chan-Paton factors of the open string. Each term in $Z(\beta)$ is described by an open string world sheet whose boundaries end on co-dimension 1 entanglement branes located at the stretched entangling surface. We will also derive the modular Hamiltonian for the open strings, thereby giving a stringy generalization of equation 8 . 


\section{Chapter II}

\section{Entanglement temperature and entanglement entropy of excited states}

\subsection{Introduction and summary}

For a spherical region $A$ in a CFT, Casini, Huerta and Myers [17] showed that the modular Hamiltonian can be expressed in terms of the physical energy density $T_{00}$ :

$$
H_{A}=\int_{A} \beta(x) T_{00}(x) .
$$

The starting point of our story is an elementary derivation of the above formula using

the representation of the ground state reduced density matrix $\left\langle\phi\left|\rho_{A}\right| \phi^{\prime}\right\rangle$ as a Euclidean Path integral integral with boundary conditions for the fields $\phi$ and $\phi^{\prime}$ along the cut at $A[51]$.

Deferring the explicit derivation for the next section, let us first discuss the basic idea. Treating $\rho_{A}$ as a propagator, we derive the expression (1) by performing the path integral along a Euclidean "time" $s$ that evolves the upper edge of A to the bottom. The resulting path integral may be expressed as:

$$
\rho_{A}=Z_{A}^{-1} T \exp \left\{-\int_{s_{i}}^{s_{f}} K(s) d s\right\}
$$


where $T$ denotes "time" ordering in $s$ and $K$ is the quantum operator generating $s$ evolution.

If the path integral of our theory is invariant under translations in $s$, then $K$ is a conserved charge independent of "entanglement time" $s$. Hence:

$$
\rho_{A}=\frac{\exp \left(-\left(s_{f}-s_{i}\right) K\right)}{Z_{A}}
$$

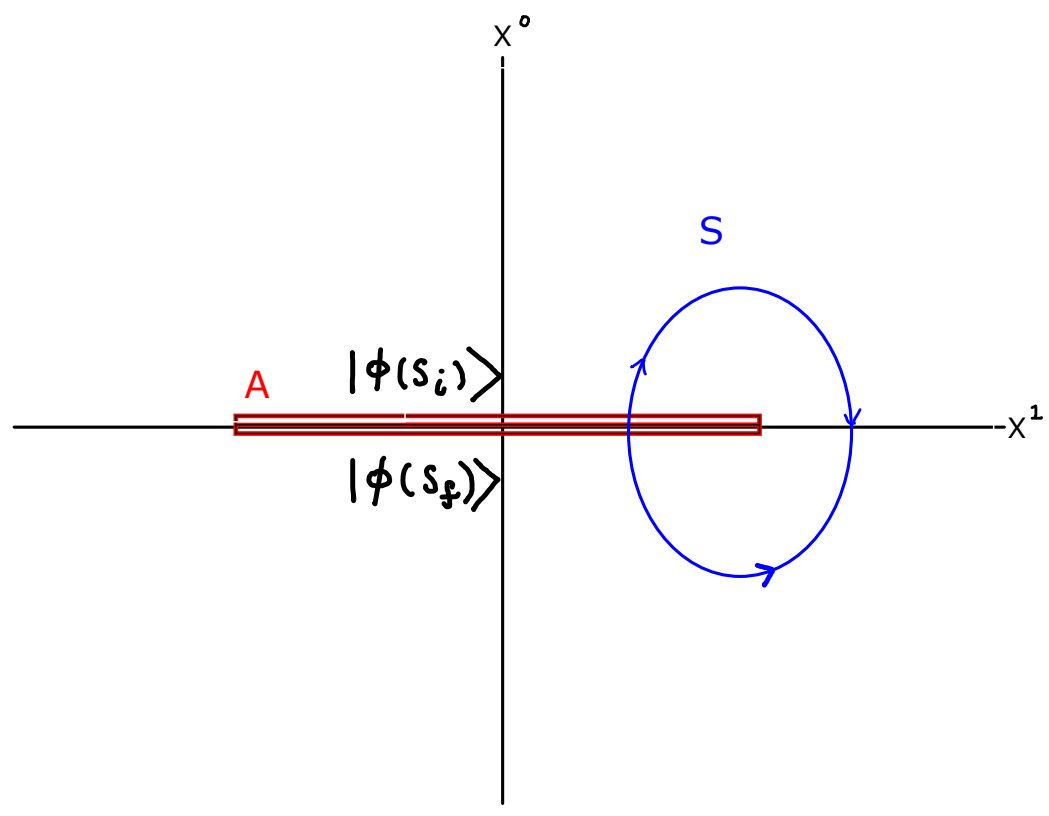

Figure 2.1: Evaluating $\rho_{A}$ along Euclidean time s

A well studied situation is the case where the theory is rotationally invariant, and $A=\left\{x^{1}>0\right\}$ is a half space. Taking $s$ to be the angular variable on the $\left(x^{1}, x^{0}=t_{E}\right)$ plane, we find the standard result that $K$ is the angular momentum operator (or the boost generator in Minkowski signature) [86].

From a more general perspective, $K$ can be viewed as a Killing energy that can be written in terms of the energy momentum tensor. For any constant $s$ slice $\Sigma$ we 
can write

$$
K=\int_{\Sigma} T_{a b} k^{a} d \Sigma^{b}, \quad H_{A}=\left(s_{f}-s_{i}\right) K
$$

where $k^{a}=\frac{d x^{a}}{d s}$ is the Killing vector for the boost and $\left\{x^{a}\right\}$ is a set of flat space coordinates. Choosing to evaluate $K$ on $\Sigma=A$ we find $k^{a} \sim \delta_{0}^{a}$ and $\Sigma^{a} \sim \delta_{0}^{a}$, which reproduces the relation (1).

Given a spherically symmetric region $A^{\prime}$ in a Euclidean CFT of any dimension, we will determine the modular Hamiltonian for $\rho_{A^{\prime}}$ by making use of a conformal map $u$ taking $A$ to $A^{\prime}$, which induces a mapping $\rho_{A} \rightarrow \rho_{A^{\prime}}=U \rho_{A} U^{-1}{ }^{1}$. The vector field $k^{\prime a}=\frac{d x^{a}}{d s^{\prime}}$ for the new entanglement time $s^{\prime}$, is just the image of $k$ under $u$. Thus, the modular Hamiltonian for $A^{\prime}$ is given by (1) with

$$
\beta(x)=2 \pi k^{\prime 0}(x)
$$

where $x \in A$ and the factor of $2 \pi$ is simply $s_{f}-s_{i}$. We will interpret $\beta(x)$ as a local inverse "entanglement" temperature, that is determined by the shape of $A$ and the background geometry of the CFT. In this interpretation, equation (1) resembles a density matrix for the original, physical system in local thermal equlibrium with temperature $\beta(x)$. The entanglement entropy is the thermal entropy of this system. It must be emphasized, however, that the appearance of $\beta(x)$ does not correspond to a "real" temperature in the sense that all inertial observers will find that local observables are at their vacuum values in accordance with $(3)^{2}$. However, the point of view of a local "entanglement temperature" is appealing: indeed $\beta(x)$ must vanish at the boundary of the region, signaling a high effective temperature close to the boundary. This behavior may be understood as the statement that the degrees of

\footnotetext{
${ }^{1}$ This is essentially a Euclidean version of the arguments in [17].

${ }^{2}$ However, non-inertial observers whose proper time coincides with $s$ will observe thermal radiation due to the local temperature [86].
} 
freedom close to the boundary are the ones most entangled with the external region, and thus have a larger entropy.

Consistent with this interpretation, we have checked that for two dimensional CFT's in various backgrounds with central charge $c$, the ground state entanglement entropy can be obtained by integrating the equilibrium thermal entropy per unit length

$$
\frac{d S_{\text {thermal }}}{d x}=\frac{c \pi}{3 \beta(x)}
$$

over the region $A$ using (5). Moreover, for excited states $\beta(x)$ relates the increase in entanglement entropy to an increase in energy inside $A$ via a local first law-like equation:

$$
d \delta S_{A}(x)=\beta(x) \operatorname{Tr}_{A}\left(\delta \rho_{A} T_{00}\right) d x
$$

Here $\frac{d \delta S_{A}}{d x}(x)$ is the local entanglement entropy density ${ }^{3}$ relative to the ground state and $\delta \rho_{A}$ is the variation in the reduced density matrix due to the increase in energy. To first order in $\delta \rho_{A}$, the total increase in entanglement entropy is obtained by integrating (7) over $A$.

Under a general variation of the ground state $\rho_{A} \rightarrow \rho_{A}+\delta \rho_{A}$ we find that the first order change in entanglement entropy is

$$
\delta S_{A}=\operatorname{Tr}_{A}\left(\delta \rho_{A} H_{A}\right)
$$

For ground states with other conserved charges $Q_{a}$ that preserve conformal invariance (e.g. momentum in $1+1 \mathrm{D})$, the corresponding charge densities $q_{a}$ and the associated chemical potentials $\mu_{a}$ will appear in the form

$$
H_{A}=\int_{A} \beta(x)\left(T_{00}-\mu_{a} q_{a}\right)
$$

\footnotetext{
${ }^{3}$ This is not to be confused with the "entanglement density", introduced in [68] and discussed later in this paper.
} 
leading to a generalized first law:

$$
d \delta S_{A}(x)=\beta(x) \delta\left\langle T_{00}\right\rangle d x-\beta(x) \mu_{a} \delta\left\langle q_{a}\right\rangle d x
$$

While preparing this manuscript, a paper [67] was posted where a set of constraint equations for $\delta S_{A}$ and an expression for "entanglement density" were derived using AdS/CFT. In section (2.6) we provide a CFT derivation of those results in two spacetime dimensions and generalize the constraint equations to arbitrary dimensions ${ }^{4}$. We will also comment on the relation between our results and those in calculations in [8] and [6].

\subsection{Path Integral Derivation of the modular Hamiltonian}

Consider a Euclidean QFT on a manifold $M$ and some spatial region $A$. The path integral expression for the reduced density matrix on $A$ is similar to the propagator of the theory except that the initial and final states live on the upper and lower edge of a branch cut defined along $A$. Thus, to switch to a canonical description, it is natural to choose a foliation of $M$ by constant $s$-slices $\Sigma(s)$ such that the initial/final slice at "time" $\left(s_{i}, s_{f}\right)$ lie on the branch cut (see Fig. 2.1). The manifold $M$ is then parametrized by coordinates $\left(s, y^{a}\right)$ where $y^{a}$ are coordinates on $\Sigma$. The reduced density matrix on $A$ in the Schrödinger picture is

$$
\left\langle\phi_{0}\left(s_{f}\right)\left|\rho_{A}\right| \phi_{0}^{\prime}\left(s_{i}\right)\right\rangle=\int D[\phi] e^{-\mathcal{S}[\phi]} \delta\left[\phi\left(s_{f}\right)-\phi_{0}\left(s_{f}\right)\right] \delta\left[\phi\left(s_{i}\right)-\phi_{0}\left(s_{i}\right)\right]
$$

where $\mathcal{S}[\phi]$ is the action functional. To find the modular Hamiltonian, we divide the "time" interval $\left[s_{i}, s_{f}\right]$ into small steps $\left[s_{n+1}, s_{n}\right]$ of size $\Delta s$ and consider a discretization of the path integral in (11). For notational simplicity we will write

\footnotetext{
${ }^{4}$ The constraint equation was recently generalized to holographic CFT's in 3 space-time dimensions in $[7]$
} 
$\rho_{A}\left[s_{n+1}, s_{n}\right]=\left\langle\phi\left(s_{n+1}\right)\left|\rho_{A}\right| \phi\left(s_{n}\right)\right\rangle$, so that

$$
\left\langle\phi_{0}\left(s_{f}\right)\left|\rho_{A}\right| \phi_{0}^{\prime}\left(s_{i}\right)\right\rangle=\int d\left[\phi\left(s_{N-1}\right)\right] \ldots d\left[\phi\left(s_{2}\right)\right] \rho_{A}\left[s_{f}, s_{N-1}\right] \ldots \rho_{A}\left[s_{n+1}, s_{n}\right] \ldots \rho_{A}\left[s_{2}, s_{i}\right]
$$

Next we will regard the matrix element $\rho_{A}\left[s_{n+1}, s_{n}\right]$ as a function of the final time $s_{f}$ and final field configuration $\phi\left(s_{n+1}, y\right)$. We wish to show that this function satisfies a heat equation

$$
\frac{\partial}{\partial s_{n+1}} \rho_{A}\left(s_{n+1}\right)=-K\left(s_{n+1}\right) \rho_{A}\left(s_{n+1}\right)
$$

and identify the operator $K\left(s_{n+1}\right)$. For a given field configuration in the path integral we need to evaluate $\frac{\partial}{\partial s_{n+1}} \mathcal{S}\left[\phi\left(s_{n+1}, y\right), s_{n+1}\right]$ at fixed $\phi\left(s_{n+1}, y\right)$. One way of doing this is to keep the final time at $s_{n+1}$, but transform the background metric by a diffeomorphism that enlarges the proper size of the integration region. Explicitly we want a coordinate transformation $s \rightarrow s^{\prime}(s)$ such that

$$
\mathcal{S}+d \mathcal{S}=\int_{s_{n}}^{s_{n+1}+d s} d s \int_{\Sigma(s)} d^{d-1} y \mathcal{L}\left[g_{a b}, \phi\right]=\int_{s_{n}}^{s_{n+1}} d s^{\prime} \int_{\Sigma\left(s^{\prime}\right)} d^{d-1} y \mathcal{L}\left[g_{a b}+d g_{a b}, \phi\right]
$$

where $g_{a b}(s, y)$ is the metric on $M$. Therefore,

$$
d \mathcal{S}=\int_{s_{n}}^{s_{n+1}} d s^{\prime} \int_{\Sigma\left(s^{\prime}\right)} d^{d-1} y \frac{\delta \mathcal{L}}{\delta g_{a b}} d g_{a b}
$$

In a general coordinate system this transformation and the response of the path integral $\left.\rho_{(} s_{n+1}\right)$ is

$$
\begin{gathered}
x^{a} \rightarrow x^{a}=x^{a \prime}-\epsilon^{a} \\
d \rho\left(s_{n+1}\right)=-\frac{1}{2} \int_{\left[s_{n}, s_{n+1}\right] \times \Sigma}\left\langle T_{a b}\right\rangle \nabla^{(a} \epsilon^{b)} \sqrt{g} d^{d} x=\int_{\Sigma\left(s_{n+1}\right)}\left\langle T_{a b}\right\rangle \epsilon^{b} d \Sigma^{a} .
\end{gathered}
$$


Here \langle\rangle refers to the path integral average on $\left[s_{n}, s_{n+1}\right]$. In the last equality we assumed the quantum conservation law $\nabla^{a}\left\langle T_{a b}\right\rangle=0$ and applied the divergence theorem; this means that $T_{a b}$ includes a possible anomalous contribution due to the transformation of the Jacobian in the path integral measure. The coordinate transformation that will satisfy equation 14 is

$$
\epsilon^{a}=\frac{d x^{a}}{d s} f(s) d s
$$

where the function $f(s)$ smoothly goes from 0 to 1 as $s$ goes from $s_{n}$ to $s_{n+1}$. This is so that we do not change the lower endpoint of the $s$ integration.

Defining

$$
K\left(s_{n+1}\right)=\int_{\Sigma\left(s_{n+1}\right)}\left\langle T_{a b}\right\rangle \frac{d x^{b}}{d s} d \Sigma^{a}
$$

we find

$$
\begin{aligned}
\frac{\partial}{\partial s_{n+1}} \rho_{A}\left[s_{n+1}, s_{n}\right] & =\int D[\phi] e^{-S[\phi]}\left(-K\left(s_{n+1}\right)\right) \\
& =\left\langle\phi_{0}\left(s_{n+1}\right)\left|-K\left(s_{n+1}\right) \rho_{A}\right| \phi_{0}^{\prime}\left(s_{n}\right)\right\rangle=-\left(\hat{K} \rho_{A}\right)\left(s_{n+1}\right) .
\end{aligned}
$$

The solution to this heat equation with initial condition $\rho_{A}\left(s_{n}\right)=0$ is $\rho_{A}\left[s_{f}, s_{N-1}\right]=$ $\left\langle\phi\left(s_{n+1}\right)|1-\Delta s K| \phi\left(s_{n}\right)\right\rangle$. Inserting this into equation (12) gives

$$
\begin{aligned}
\left\langle\phi_{0}\left(s_{f}\right)\left|\rho_{A}\right| \phi_{0}^{\prime}\left(s_{i}\right)\right\rangle & =\int \prod_{n=1}^{N-1} D\left[\phi\left(s_{n}\right)\right]\left\langle\phi\left(s_{n+1}\right)|1-\Delta s K| \phi\left(s_{n}\right)\right\rangle \\
& =\left\langle\phi_{0}\left(s_{f}\right)\left|T \exp \left(-\int_{s_{i}}^{s_{f}} K(s) d s\right)\right| \phi_{0}^{\prime}\left(s_{i}\right)\right\rangle .
\end{aligned}
$$

This is the most general form of the reduced density matrix in a QFT. Since equation (22) only depends on the geometric data provided by the vector field $\frac{d x^{a}}{d s}$ which in turn is determined by the region $A$, it represents a universal relation between the reduced density matrix and the quantum stress tensor. 
To recover the local modular Hamitonian (1), we consider regions $A$ for which $s \rightarrow s+d s$ is a spacetime symmetry of the path integral (11) so that $K[s]$ is the corresponding conserved charge. Since $K$ is independent of $s$, we can evaluate it on any time slice (say at $s_{i}$ ) and the time ordered product in $(22)$ reduces to

$$
\rho_{A}=Z_{A}^{-1} \exp \left(-\left(s_{f}-s_{i}\right) K\left(s_{i}\right)\right)
$$

Below we will show that $s \rightarrow s+d s$ is indeed a spacetime symmetry of the path integral if $A$ is a half space in a rotationally invariant QFT or a spherical region in a CFT, and we will derive the corresponding local modular Hamiltonians. Here we would like to note that given a small deformation of the region $A$ away from translational or spherical symmetry, one could perform a systematic expansion of equation (22) using the deformed modular Hamiltonian $K_{0}+\epsilon K_{1}$. To first order in $\epsilon$ this would just add a perturbation to the local modular Hamiltonian which is localized near the

boundary of $A$. A similar strategy can be applied to deformations of the theory away from rotational or conformal invariance.

\subsection{Examples of local modular Hamiltonians}

\subsection{1 modular Hamiltonians in 2D}

To illustrate how to compute $K$ and its entanglement entropy, we first review the case of a rotationally invariant $\mathrm{QFT}$ on $\mathbb{R}^{2}$ with the region $A$ being the half line $A=\left\{x^{1}>0\right\}$ [85]. Since $A$ is mapped to itself by a $2 \pi$ rotation, we choose $s$ to be the angular coordinate on the Euclidean plane so that $\Sigma(s)$ are rays emanating from the origin as in Figure 2.2.

Then

$$
\frac{d x^{a}}{d s} \partial_{a}=x^{1} \partial_{0}-x^{2} \partial_{1}
$$




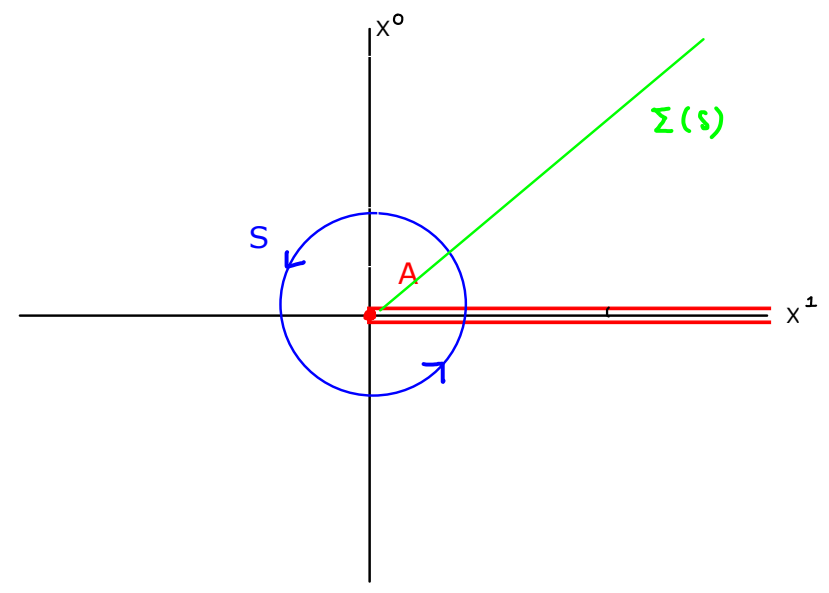

Figure 2.2: Foliation of the Euclidean plane corresponding to angular quantization

is a Killing vector field generating rotations of the plane. Since the path integral measure is assumed to be rotationally invariant, $K$ is just the angular momentum $[79]$

$$
K=\int_{\Sigma(s=0)} x^{1} T_{00}-x^{0} T_{01}=\int_{A} x^{1} T_{00} .
$$

The modular Hamiltonian is given by equation (1) with the entanglement temperature

$$
\beta=2 \pi x^{1}
$$

Upon Wick rotating $s \rightarrow i s$, the circular flow generated by $K$ becomes hyperbolas representing the worldlines of uniformly accelerated observers, and $\beta(x)$ is the proper temperature they experience. Thus in Minkowski signature $K$ is the boost generator. The form of the modular Hamiltonian implies that $\rho_{A}$ represents an ensemble with the physical energy density $T_{00}$ in local thermal equilibrium with local temperature $\beta(x)$; its entanglement entropy is therefore just the thermal entropy, obtained by integrating the thermal entropy density $\frac{d S_{\text {thermal }}}{d x}$ over $A[79]$.

In particular, for a CFT with central charge $c$, it is well known that [51]

$$
\frac{d S_{\text {thermal }}}{d x}=\frac{c \pi}{3 \beta(x)}
$$


so the entanglement entropy is

$$
S_{A}=\int_{\delta}^{L} d x \frac{c \pi}{6 x}=\frac{c}{6} \log \frac{L}{\delta}
$$

where we have introduced a UV and IR cutoff on $A$ restricting the integration to $[\delta, L]$. The local temperature is higher near the boundary of $A$ and diverges at $x=0$ due to the zero of the vector field, which is also the singularity of the foliation defined by $s$. As a result, most of the contribution to the entanglement entropy arises from near the edge.

For a $\mathrm{CFT}$ on $\mathbb{R}^{2}$ we can easily generalize the previous results to an arbitrary interval $A^{\prime}=[u, v]$. Let $z=x^{1}+i x^{0}$ so that $\frac{d z}{d s}=i z$ is the rotational vector field appropriate to the region $A$ discussed previously. The conformal map

$$
z=-\frac{w-u}{w-v}
$$

induces a transformation $U$ on the reduced density matrices:

$$
\rho_{A} \rightarrow \rho_{A^{\prime}}=U \rho_{A} U^{-1}
$$

by transforming the boundary conditions of the path integral. The path integral measure is conformally invariant because there is no anomaly in flat space. Meanwhile, the vector field $\frac{d z}{d s^{\prime}}$ is mapped to

$$
\frac{d w}{d s^{\prime}}=\frac{d w}{d z} \frac{d z}{d s^{\prime}}=\frac{i(w-u)(w-v)}{u-v}
$$

It is clear that the periodic flow defined by this vector field will evolve $A^{\prime} \rightarrow A^{\prime}$. Moreover, the transformation $w \rightarrow w+\frac{d w}{d s^{\prime}} d s^{\prime}$ is a symmetry of the CFT on the $w$ 


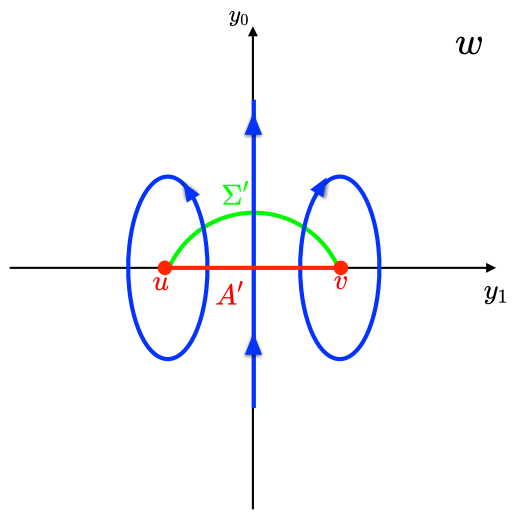

$w$

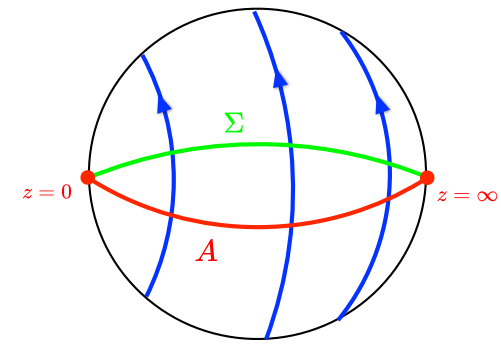

Figure 2.3:

A rotation on the $\mathrm{z}$ plane (represented as a Riemann sphere) is mapped to a conformal rotation on the $w$ plane

plane, because it can be decomposed into a combination of a conformal transformation between $z$ and $w$, and an ordinary rotation on the $z$ plane.

Thus, the modular Hamiltonian for $\rho_{A^{\prime}}$ is

$$
H_{A^{\prime}}=\int_{A} 2 \pi \frac{\left(y^{1}-u\right)\left(y^{1}-v\right)}{u-v} T_{00} d y
$$

where we defined $w=y^{1}+i y^{0}$ and evaluated the integral along $A$ for convenience. As before, the entanglement entropy is obtained from integrating $\frac{d S_{\text {thermal }}}{d x}$ using the entanglement temperature

$$
\beta(y)=2 \pi \frac{\left(y^{1}-u\right)\left(y^{1}-v\right)}{u-v} .
$$

This gives

$$
S_{A^{\prime}}=\frac{c}{3} \log \frac{v-u}{\delta}
$$

as expected ${ }^{5}$.

${ }^{5}$ Note that even though $\operatorname{Tr}_{A}\left(\rho_{A} \log \rho_{A}\right)$ is invariant under the similarity transformation (30) of the reduced density matrix, we get a different result for the entanglement entropy of $\rho_{A^{\prime}}$ because we have to transform the regularized boundary of $A$. 
For a CFT at finite temperature $\left(w \sim w+i \beta^{\prime}\right)$ or on a spatial circle $(w \sim w+L)$, we can similarly derive the modular Hamiltonian by finding the conformal map from the $z$-plane to $\mathbb{R} \times S^{1}$ or $S^{1} \times \mathbb{R}$. Given $A^{\prime}=[-l, l] \times\{0\}$, the conformal map and entanglement temperature for a CFT at the (ordinary) temperature $\beta^{\prime}$ is

$$
z=\frac{-\exp \left(\frac{2 \pi w}{\beta^{\prime}}\right)+\exp \left(-\frac{2 \pi l}{\beta^{\prime}}\right)}{\exp \left(\frac{2 \pi w}{\beta^{\prime}}\right)-\exp \left(\frac{2 \pi l}{\beta^{\prime}}\right)}, \quad \beta=2 \beta^{\prime} \operatorname{csch}\left(\frac{2 l \pi}{\beta^{\prime}}\right) \sinh \left(\frac{\pi(l-y)}{\beta^{\prime}}\right) \sinh \left(\frac{\pi(l+y}{\beta^{\prime}}\right) .
$$

The results for a CFT at finite size can be obtained from equation (35) by the substitution $\beta^{\prime} \rightarrow i L$. Below we summarize the results for the entanglement temperature and entanglement entropy obtained by integrating the thermal entropy density in various CFT backgrounds.

Table 2.1: Entanglement Temperature for $A=[-l, l]$ in different CFT backgrounds

\begin{tabular}{|l|c|c|}
\hline CFT Background & Entanglement Temperature $\beta(y)$ & Entropy $S_{A}=\int_{A} \frac{\pi c}{3 \beta(y)}$ \\
\hline$M=\mathbb{R}^{2}$ & $\frac{2 \pi\left(l^{2}-y^{2}\right)}{2 l}$ & $\frac{c}{3} \ln \frac{l}{\delta}$ \\
\hline$M=\mathbb{R} \times S^{1}$ & $2 \beta^{\prime} \operatorname{csch}\left(\frac{2 l \pi}{\beta^{\prime}}\right) \sinh \left(\frac{\pi(l-y)}{\beta^{\prime}}\right) \sinh \left(\frac{\pi(l+y)}{\beta^{\prime}}\right)$ & $\frac{c}{3} \ln \left(\frac{\beta^{\prime}}{\pi \delta} \sinh \left(\frac{2 \pi l}{\beta^{\prime}}\right)\right)$ \\
\hline$M=S^{1} \times \mathbb{R}$ & $2 L \csc \left(\frac{2 l \pi}{L}\right) \sin \left(\frac{\pi(l-y)}{L}\right) \sin \left(\frac{\pi(l+y)}{L}\right)$ & $\frac{c}{3} \ln \left(\left(\frac{L}{\pi \delta} \sin \left(\frac{2 \pi l}{L}\right)\right)\right.$ \\
\hline
\end{tabular}

The results for the entanglement entropies were derived previously using the replica trick, $[13,14,51,60]$, and serve as a check on our results for the entanglement temperature and Hamiltonian.

\subsubsection{Modular Hamiltonians in higher dimensions}

Here we generalize the results of the previous section to spherical entangling surfaces in dimensions $d>2$. As before, we first consider a rotationally invariant CFT on $\mathbb{R}^{d}$ with $A=\left\{x^{1}>0\right\}$. We choose polar coordinates on the $x^{1}, x^{0}$ plane $x^{1}=z \cos \left(\frac{s}{l}\right)$, 
$x^{0}=z \sin \left(\frac{s}{l}\right)$, so the flat metric is

$$
d \tau^{2}=\left(\frac{z}{l}\right)^{2} d s^{2}+d z^{2}+d \vec{x}^{2}
$$

At this point $l$ is an arbitrary length parameter introduced to make $s$ dimensionful. Then the result (25) for the modular Hamiltonian of $\rho_{A}$ is still valid. Now we map $\mathbb{R}^{d} \rightarrow H^{d-1} \times S^{1}$, by multiplying the metric above by a conformal factor $\left(\frac{l}{z}\right)^{2}$.

$$
d \tau_{H^{d-1} \times S^{1}}^{2}=d s^{2}+\left(\frac{l}{z}\right)^{2}\left(d z^{2}+d \vec{x}^{2}\right)
$$

The $H^{d-1}$ factor refers to hyperbolic space, which is the image of the half space $A$. Thus we see that $\rho_{A}$ is transformed into a thermal density matrix $\rho_{H^{d-1}}$ on hyperbolic space. Since this conformal map does not change the original coordinates on $\mathbb{R}^{d}$, the vector field generated by the new modular Hamiltonian is just $\frac{\partial}{\partial s}$.

Now consider a new reduced density matrix $\rho_{A^{\prime}}$ for a ball of radius $l$. We will obtain the modular Hamiltonian $H_{A^{\prime}}$ by mapping $\rho_{H^{d-1}} \rightarrow \rho_{A^{\prime}}$ as follows. First we choose coordinates $\left(u, \Omega_{d-2}, s\right)$ on $H^{d-1} \times S^{1}$ and spherical coordinates $\left(r, \Omega_{d-2}, t\right)$ on $\mathbb{R}^{d}$ so that the metrics are

$$
\begin{array}{r}
d \tau_{H^{d-1} \times S^{1}}^{2}=d s^{2}+R^{2}\left(d u^{2}+\sinh (u)^{2} d \Omega_{d-2}^{2}\right), \\
d \tau_{R^{d}}^{2}=d t^{2}+d r^{2}+r^{2} d \Omega_{d-2}^{2} .
\end{array}
$$

Then, defining complex coordinates $\sigma=u+i \frac{s}{l}$ and $w=r+i t$ on the respective two dimensional slices, we consider the mapping introduced in [54]

$$
e^{-\sigma}=\frac{l-w}{l+w} .
$$


This is an analogue of equation (29) mapping $\rho_{A^{\prime}} \rightarrow \rho_{H^{d-1}}$. The entanglement vector field and modular Hamiltonian is

$$
\frac{d w}{d s}=\frac{d w}{d \sigma} \frac{d \sigma}{d s}=i \frac{l^{2}-r^{2}}{2 l}, \quad H_{A}=2 \pi \int_{A} \frac{l^{2}-r^{2}}{2 l} T_{00} .
$$

This agrees with the result of [17], where a Minkowski signature version of the conformal mapping (40) was used to derive the modular Hamiltonian.

\subsection{CFT derivation of Entanglement Entropy for excited states}

Consider a state $|\psi\rangle$ in a QFT in $\mathbb{R}^{1, d-1}$ with a density matrix $\rho^{0}=|\psi\rangle\langle\psi|$. As in [8] we make a small perturbation $\rho=\rho^{0}+\delta \rho$ and consider the entanglement entropy of a region $A$. Expanding to first order in $\delta \rho_{A}$ we find

$$
S_{A}=-\operatorname{Tr}_{A}\left(\rho_{A} \ln \rho_{A}\right)=-\operatorname{Tr}_{A}\left(\rho_{A}^{0} \ln \rho_{A}^{0}\right)-\operatorname{Tr}_{A}\left(\delta \rho_{A}^{0} \ln \rho_{A}^{0}\right)-\operatorname{Tr}_{A}\left(\delta \rho_{A}\right),
$$

where $\delta \rho_{A}=\operatorname{Tr}_{B}(\delta \rho)$. The normalization $\operatorname{Tr}\left(\rho_{A}\right)=\operatorname{Tr}_{A}\left(\rho_{A}^{0}\right)=1$ implies $\operatorname{Tr}\left(\delta \rho_{A}\right)=$ 0 , so the first order change in entanglement entropy due to the perturbation $\delta \rho$ is simply

$$
\delta S_{A}=-\operatorname{Tr}_{A}\left(\delta \rho_{A} \ln \rho_{A}^{0}\right)=\operatorname{Tr}_{A}\left(\delta \rho_{A} H_{A}\right)
$$

Note that there is also a term proportional to $\operatorname{Tr}(\delta \rho)$ which vanishes due to the normalization $\operatorname{Tr}(\rho)=1$. When the state $\rho^{0}$ is the ground state, we will refer to $\delta S_{A}$ as the renormalized entanglement entropy ${ }^{6}$ [51]. It is just the increase in "entanglement energy" of the new state, measured according to the ground state modular Hamiltonian. However we emphasize that equation (43) applies to an arbitrary deformation

\footnotetext{
${ }^{6}$ This is only a first order approximation to the renormalized entropy, but we will just call it renormalized entropy for short.
} 
$\delta \rho$ for any initial state $\rho^{0}$. When the region $A$ is a half space in a QFT or a spherical ball in a CFT, we can use the entanglement temperatures previously derived to obtain $H_{A}$ for the ground state as in equation (1). From equation (43) we have:

$$
\delta S_{A}=\operatorname{Tr}_{A}\left(\delta \rho_{A} \int_{A} \beta(x) T_{00}(x)\right)=\int_{A} \beta(x) \operatorname{Tr}\left(\delta \rho T_{00}(x)\right):=\int_{A} \beta(x) \delta\left\langle T_{00}(x)\right\rangle
$$

In the second to last equality, we noted that the operator $T_{00}(x)$ is only being evaluated inside $A$ so that $\delta \rho_{A}$ can be replaced with $\delta \rho$. Note that in (43) the operator $\delta \rho_{A}$ and $H_{A}$ are defined on a subregion $A$ with boundaries, which implies boundary conditions have to be imposed at $\partial A$ on their quantization. On the other hand, in (44) the operator $T_{00}$ is interpreted as the energy density quantized with the boundary conditions appropriate to the whole space; we have merely chosen to evaluate it inside $A$. These two interpretations must agree by the definition of the reduced density matrix. As a check, in Appendix B we will show that for a particular excitation of a free scalar field with non-uniform energy density, (44) and (43) do indeed give the same result for $\delta S_{A}$.

When $\delta\left\langle T_{00}\right\rangle$ is spatially uniform ${ }^{7}$ inside $A$, we can remove it from the integration, so that

$$
\delta S_{A}=\beta_{0} \delta\left\langle T_{00}\right\rangle \operatorname{Vol}(A):=\beta_{0} \delta E_{A}
$$

where $\delta E_{A}=\delta\left\langle T_{00}\right\rangle \operatorname{Vol}(A)$ is the excitation energy inside region $A$, and $\beta_{0}$ is the average entanglement temperature inside $A$

$$
\beta_{0}=\frac{\int_{A} \beta(x)}{\operatorname{Vol}(A)}
$$

\footnotetext{
${ }^{7}$ Since our modular Hamiltonian was derived for a CFT on $\mathbb{R}^{d}$, we will assume the energy density starts to die off somewhere outside $A$, in order for the energy to be finite.
} 
When the region $A$ has radius $l$, we find ${ }^{8} \beta_{0}=\frac{2 \pi}{d+1} l$ in agreement with the result of [6]. However, we note that the holographic results of [6] only strictly apply to nonabelian gauge theories with holographic duals, at large $\mathrm{N}$ and assuming a small region $A$ (i.e. for small radius $l$ ), whereas our result is valid to order $O(\delta \rho)$ for any CFT and any radius $l$. We also note that there is a discrepancy between our results when $\delta\left\langle T_{00}\right\rangle$ is spatially varying. Given a state with $\delta\left\langle T_{00}\right\rangle=\sum_{n=0}^{\infty} a_{n} r^{n}$ in a $d>2$ dimensional $\mathrm{CFT}^{9}$, we find

$$
\delta S_{A}=2 \pi \operatorname{Vol}\left(S^{d-2}\right) \sum_{n=0} \frac{a_{n} l^{d+n}}{(d+n)^{2}-1}
$$

which disagrees with the holographic calculation of the same quantity in equation (20) of [6]. In section IV, we will discuss the holographic version of eq 43 and speculate on a possible source of the discrepancy. As noted earlier, we have checked in appendix B that our results (43) and (44) are consistent for a non-uniform excitation of a free scalar field, where $\delta S_{A}$ can be computed explicitly.

\subsection{A generalized first law for entanglement entropy}

Equation (44) resembles a local first law of thermodynamics inside the region $A$ :

$$
d \delta S_{A}(x)=\beta(x) \delta\left\langle T_{00}(x)\right\rangle d x
$$

When other conserved charges are present, a generalization of equation (48) can be derived as follows. Consider a state at finite temperature $T$ and with conserved charges $Q_{a}$ that preserve conformal invariance and chemical potentials $\mu_{a}$ weighted

\footnotetext{
${ }^{8}$ As already noted in [6], this is also consistent with the computation of $\delta S_{A}$ for primary states of a two dimensional CFT which was performed in [5] via the replica trick.

${ }^{9}$ We will explain the restriction to $d>2$ in the section VI.
} 
with the following density matrix

$$
\rho=\frac{\exp \left(-\frac{\left(H-\mu_{a} Q_{a}\right)}{T}\right)}{Z} .
$$

After tracing over the complement of $A$ we arrive at a path integral representation of $\rho_{A}$ similar to the one given in equation (11), except that adding the charges has effectively shifted our Hamiltonian from $H$ to $H^{\prime}=H-\mu_{a} Q_{a}$. The corresponding shift in the energy density is $T_{00}^{\prime}=T_{00}-\mu_{a} q_{a}$, where we introduced the charge densities $q_{a}$ by $Q_{a}:=\int_{\text {space }} q_{a} d^{d-1} x$. Going through the same path integral derivation as in section 2.2, we would reproduce equation (1) with $T_{00}$ replaced by $T_{00}^{\prime}$. Under a deformation $\delta \rho$ that changes the charge densities and energy inside $A$, equation (48) now becomes

$$
d \delta S_{A}(x)=\beta(x) \delta\left\langle T_{00}^{\prime}(x)\right\rangle d x=\beta(x)\left\{\delta\left\langle T_{00}(x)\right\rangle d x-\mu_{a} \delta\left\langle q_{a}(x)\right\rangle d x\right\}
$$

A simple way to check the above argument for the modular Hamiltonian leading to equation (50) is to consider a state $\rho \sim \exp \left[-\beta^{\prime}(H-\mu P)\right]$ for a two dimensional CFT with total central charge $c$. In this case the conserved Virasoro charges are the Hamiltonian $H=L_{0}+\bar{L}_{0}-\frac{c}{12}$ and momentum $P=L_{0}-\bar{L}_{0}$. The modular Hamiltonian for an interval $A=[0, l]$ is

$$
H_{A}=\int_{0}^{l} \beta(x)\left(T_{00}-\mu T_{01}\right) d x=\int_{0}^{l} \beta(x)(1-\mu) T_{++}+\beta(x)(1+\mu) T_{--},
$$

where $T_{ \pm \pm}=\frac{1}{2}\left(T_{00} \pm T_{01}\right)$ are the right and left moving components of the stress tensor, and $\beta(x)$ is the entanglement temperature (35) for a CFT at finite temperature ${ }^{10} \beta^{\prime}$. The operator in equation (51) is the sum of two commuting modular Hamiltonians

\footnotetext{
${ }^{10}$ Technically, to get a discrete spectrum for $P$ we should put the CFT on a spatial $S^{1}$ of length $L$. Here we will assume $\beta^{\prime}>>L$, so that we can ignore the periodicity along $L$ in computing the entanglement temperature.
} 
corresponding to non-interacting ensembles at finite (ordinary) temperature $\beta_{ \pm}=$ $\beta^{\prime}(1 \pm \mu)$ and with energy density $T_{ \pm \pm}$. Assuming that the left and right central charges are equal, each ensemble has an effective central charge of $\frac{c}{2}$. Thus the entanglement entropy is:

$$
S_{A}=\frac{c}{6} \ln \left(\frac{\beta_{+}}{\pi \delta} \sinh \left(\frac{\pi l}{\beta_{+}}\right)\right)+\frac{c}{6} \ln \left(\frac{\beta_{-}}{\pi \delta} \sinh \left(\frac{\pi l}{\beta_{-}}\right)\right)
$$

This agrees with the result of [53] obtained via the replica trick and holographic calculations.

\subsection{Holographic derivation and discussion of related papers}

According to the holographic prescription of [72], the entanglement entropy for a state $|\psi\rangle$ in a region $A$ of a $d$-dimensional CFT with a holographic dual gravity theory is

$$
S_{A}=\frac{\operatorname{Area}\left(\gamma_{A}\right)}{4 G}
$$

where $\gamma$ is a minimal surface, anchored on $\partial A$, in the bulk spacetime representing the gravity dual of the corresponding CFT, $G$ is the bulk Newton's constant. The geometry dual to the ground state in the CFT corresponds to pure AdS

$$
d \tau^{2}=\left(\frac{R}{z}\right)^{2}\left(-d t^{2}+d z^{2}+r^{2} d \Omega_{d-2}^{2}\right)
$$

and the minimal surface for $A=\{r=l\}$ is a half sphere extending into the bulk: $\gamma_{A}=\left\{r^{2}=l^{2}-z^{2}\right\}$

For general excited states, it is difficult to find the exact bulk metric and compute the minimal surface. However, just as in the CFT computation of the previous section, a drastic simplification occurs if we consider only the first order deformation of the entanglement entropy, which is proportional to the variation of area functional : 


$$
\delta \operatorname{Area}\left(\gamma_{A}\right)=\delta \int_{\gamma_{A}} \sqrt{g}=\int_{\gamma_{A}} \delta \sqrt{g}
$$

In the last equality, we observed that the area variation due to the deformation of the surface $\gamma_{A}$ vanishes by the definition of a minimal surface. Thus, the area variation is entirely due to the change in the metric, and there is no need to solve for the minimal surface in the new geometry. Comparing this equation to (45), we see that $\delta \rho_{A}$ corresponds to the deformation of the metric while $H_{A}$ ( and thus $\rho_{A}$ ) corresponds to the ground state minimal surface.

The second fact is less obvious from the usual AdS/CFT correspondence, but it is consistent with ideas proposed in [17]. There it was shown that after a conformal mapping to $\mathcal{H}=H^{d-1} \times R$, the ground state $\rho_{A}$ is thermal and dual to a hyperbolic foliation of AdS with a causal horizon that is "anchored" on $\partial A$. In this setting, the minimal surface is identified with the causal horizon, and since all leaves of a foliation are diffeomorphic, the minimal surface determines the whole foliation, which in turn specifies the ground state reduced density matrix.

As in $[6]^{11}$ we consider an excited state with energy density ${ }^{12}\left\langle T_{00}\right\rangle=\frac{d R^{d-1} m}{16 \pi G}$. As established in ref. [23], the holographic stress tensor associated with this energy density and the boundary metric determines the asymptotic form of the bulk metric near the boundary at $z \sim 0$ to be:

$$
d \tau^{2}=\left(\frac{R}{z}\right)^{2}\left(-g^{-1}(z) d t^{2}+g(z) d z^{2}+r^{2} d \Omega_{d-2}^{2}\right), \quad \text { with } \quad g(z)=1+m z^{d}+\ldots
$$

\footnotetext{
${ }^{11}$ see also [1] for an extension of results in [6]

${ }^{12}$ To facilitate comparisons with [6], in this section we write $\delta\left\langle T_{00}\right\rangle=\left\langle T_{00}\right\rangle$, with the understanding that the energy density in the latter expression is normal ordered so as to subtract the vacuum energy. Note that there is a typo in eq. (2) of [6] where $d$ was replaced with $d-1$.
} 
where the ellipsis denotes higher order terms in $z$. In this approximation, the first order variation of the entanglement entropy for a spherical region $\mathrm{A}$ is

$$
\begin{aligned}
\left.\frac{\delta S_{A}^{\prime}}{\delta m}\right|_{m=0} \delta m & =\left.\frac{\delta \operatorname{Area}\left(\gamma_{A}\right)}{4 G}\right|_{m=0}=R^{d-1} \Omega_{d-2} \int_{0}^{l} \frac{r(z)^{d-2}}{z^{d-1}} \delta \sqrt{g(z)+r^{\prime}(z)^{2}} \\
& =\beta_{0} \delta E_{A}
\end{aligned}
$$

where we evaluated the integral along the half sphere $r^{2}=l^{2}-z^{2}$ corresponding to the ground state at $m=0, \beta_{0}=\frac{2 \pi}{d+1} l$, and $\delta E_{A}$ is defined as in the section IV. The notation $\delta S_{A}^{\prime}$ is a reminder of the additional approximation due to the expansion (56), where sub-leading terms in $z$ were dropped. However, in this case, this approximation (truncation) leads to a result which agrees with the field theoretic one in eq. (46)

Next, we consider a non-uniform state with energy density $\left\langle T_{00}\right\rangle=\frac{d R^{d-1} m}{16 \pi G} \sum_{n \geq 0} c_{n} r^{n}$ in a $d>2$ dimensional CFT. Note that this state is not allowed for $d=2$ spacetime dimensions, because the energy density has to satisfy a wave equation, as explained later in this section. The dual metric has the same form as in (56) with

$$
g(z)=1+m z^{d} \sum_{n \geq 0} c_{n} r^{n}+\ldots,
$$

using (55) we find:

$$
\delta S_{A}^{\prime}=\frac{m l^{d} R^{d-1} \operatorname{Vol}\left(S^{d-2}\right)}{8 G} \sum_{n \geq 0} \frac{c_{n} l^{n}}{1+d+n} .
$$

The above expression reproduces and generalizes the results in [6], without recourse to an explicit evaluation of the minimal surfaces. This time, we note that above $\delta S_{A}^{\prime}$ differs from our result (47) for the entropy of a sphere, although both are supposed to represent entropy of a system with the same non-uniform energy density.

In [6], use of equation (56) was justified by taking the small region limit, that is, the $l \rightarrow 0$ limit in which $\gamma_{A}$ approaches the $z=0$ boundary. However, neglect of 
higher order terms in $z$, while not affecting the energy density $\left\langle T_{00}\right\rangle$, may affect the computed entropy. For example, adding a correction of the form $m z^{d+k} r^{\mu}$ will yield, using (55), a contribution proportional to $l^{d+k+\mu}$ to the holographic entropy. Neglect of such terms may be the reason that our results agree with those of [6] only for the case of uniform energy density. In this way, our result provides an easy consistency check for the $z \rightarrow 0$ limit metric used in holographic calculations.

\subsubsection{Dynamical equations for entanglement entropy and entanglement density}

While this project was being completed, we noticed a recent paper [67] where a set of dynamical equations were derived for $\delta S_{A}$ in the case of time dependent excited states by using the holographic formula (53). In $d=2$ spacetime dimensions they are:

$$
\begin{aligned}
\left(\partial_{t}^{2}-\partial_{\xi}^{2}\right) \delta S_{A}(\xi, l, t) & =0 \\
\left(\frac{\partial_{l}^{2}}{4}-\frac{\partial_{t}^{2}}{4}-\frac{1}{2 l^{2}}\right) \delta S_{A}(\xi, l, t) & =0
\end{aligned}
$$

where $A=[\xi-l, \xi+l]$. In the holographic setting these equations arose from solving Einstein's equations perturbatively to determine the evolution of the metric for the excited state. Here we will provide a simple field theoretic derivation of these equations. First note that in terms of the variable $x^{\prime}=x-\xi$, the renormalized entanglement entropy for a CFT on a plane is

$$
\delta S_{A}=2 \pi \int_{-l}^{l} d x^{\prime} \frac{l^{2}-x^{\prime 2}}{2 l}\left\langle T_{00}\right\rangle\left(x^{\prime}+\xi, t\right)
$$

so the entanglement temperature is independent of $\xi$. Thus,

$$
\left(\partial_{t}^{2}-\partial_{\xi}^{2}\right) \delta S_{A}(\xi, l, t)=2 \pi \int_{-l}^{l} d x^{\prime} \frac{l^{2}-x^{\prime 2}}{2 l}\left(\partial_{t}^{2}-\partial_{\xi}^{2}\right)\left\langle T_{00}\right\rangle\left(x^{\prime}+\xi, t\right)=0
$$


where in the last equality we used the fact that in $d=2$ the conservation of the energy momentum tensor combined with its tracelessness imply that $T_{00}=T_{++}+T_{--}$is a sum of left and right movers, and therefore satisfy the wave equation. The second equation (61) can be obtained straightforwardly by applying the differential operator to $(62)$ and integrating by parts using $\partial_{t}^{2} T_{00}=-\partial_{\xi}^{2} T_{00}=-\partial_{x^{\prime}}^{2} T_{00}$. As in [67], we can also generalize and (61) to the case when we couple an operator $O(x, t)$ to a source $J(x, t)$ so that our physical Hamiltonian is deformed to $H^{\prime}=H-\int J O d^{d-1} x$. Provided that $O(x, t)$ preserves conformal symmetry, this deformation changes the ground state Hamiltonian by deforming the energy density $T_{00} \rightarrow T_{00}^{\prime}=T_{00}-J O$ in 1. The equations (60) and (61) are now modified by source terms that arise form the differential operators hitting $J(x, t) O(x, t)$. Thus

$$
\begin{array}{r}
\left(\partial_{t}^{2}-\partial_{\xi}^{2}\right) \delta S_{A}(\xi, l, t)=\int_{-l}^{l} \beta\left(x^{\prime}, l\right)\left(\partial_{t}^{2}-\partial_{\xi}^{2}\right)\left(J\left(x^{\prime}+\xi, t\right)\left\langle O\left(x^{\prime}+\xi, t\right)\right\rangle_{J}\right), \\
\left(\frac{\partial_{l}^{2}}{4}-\frac{\partial_{t}^{2}}{4}-\frac{1}{2 l^{2}}\right) \delta S_{A}(\xi, l, t)=-\int_{-l}^{l} \beta\left(x^{\prime}, l\right) \frac{\partial_{t}^{2}}{4}\left(J\left(x^{\prime}+\xi, t\right)\left\langle O\left(x^{\prime}+\xi, t\right)\right\rangle_{J}\right),
\end{array}
$$

with

$$
\beta\left(x^{\prime}, l\right)=2 \pi \frac{l^{2}-x^{\prime 2}}{2 l} .
$$

To facilitate a comparison with the result of [67], we take the Fourier transform of $\left\langle O\left(x^{\prime}+\xi, t\right)\right\rangle_{J}$ and make explicit the dependence of $J\left(x^{\prime}+\xi, t\right)$ on $\left\langle O\left(k_{1}, w_{1}\right)\right\rangle_{J}$ :

$$
\begin{array}{r}
\langle O(x, t)\rangle_{J}=\int d \omega_{1} \int d k_{1}\left\langle O\left(k_{1}, \omega_{1}\right)\right\rangle_{J} e^{i\left(k_{1} \xi+\omega_{1} t\right)} e^{i k_{1} x^{\prime}} \\
J\left(x^{\prime}+\xi, t\right)=\int d \omega_{2} \int d k_{2} f\left(k_{2}, \omega_{2}\right)\left\langle O\left(k_{2}, \omega_{2}\right)\right\rangle_{J} e^{i\left(k_{2} \xi+\omega_{2} t\right)} e^{i k_{2} x^{\prime}}
\end{array}
$$

Above we chose the source $J$ corresponding to the perturbation of the bulk scalar given in equation (3.17) of [67]. Inserting these in (64) and integrating over $x^{\prime}$ gives 
equations of the form

$$
\begin{aligned}
& \left(\partial_{t}^{2}-\partial_{\xi}^{2}\right) \delta S_{A}(\xi, l, t)= \\
& \left.\quad \int d \omega_{1} d \omega_{2} \quad d k_{1} d k_{2} F\left(k_{1}, k_{2}, \omega_{1}, \omega_{2}\right)\left\langle O\left(k_{1}, \omega_{2}\right)\right\rangle_{J}\left\langle O\left(k_{2}, \omega_{2}\right)\right\rangle_{J} e^{i\left(\left(k_{1}+k_{2}\right) \xi+\left(\omega_{1}+\omega_{2}\right)\right.} t \xi 9\right)
\end{aligned}
$$

and similarly (65). These equations have the same form as (3.22) and (3.23) of [67], which were interpreted as the holographic dual to the perturbative Einstein's equations with the right hand side serving as the matter source.

In general dimensions, we can derive a constraint equation similar to (61) for a ball $A$ of radius $l$ centered on $\vec{\xi}$ :

$$
\left(\partial_{l}^{2}-(d-2) \frac{\partial_{l}}{l}-\nabla_{\xi}^{2}-\frac{d}{l^{3}}\right) \delta S_{A}(\vec{\xi}, l, t)=0
$$

As in the case of 2 dimensions, this can be verified straightforwardly by applying the differential operator above to the expression for $\delta S_{A}$ in (62) and integrating by parts after noting that:

$$
\int_{A} \beta(r) \nabla_{\vec{\xi}}^{2} T_{00}(\vec{\xi}+\vec{r}) d r d \Omega=\int_{A} \beta(r) \nabla_{\vec{r}}^{2} T_{00}(\vec{\xi}+\vec{r}) d r d \Omega=-\int_{A} \nabla \beta(r) \cdot \nabla_{\vec{r}} T_{00}(\vec{\xi}+\vec{r}) d r d \Omega
$$

For $d=3,[7]$ recently derived the same equation holographically. In [62], a general argument was proposed explaining why (62) leads to the perturbative Einstein's equations via the holographic entanglement entropy formula (53). In addition, a quantity called entanglement density was introduced in [67]. In $d=2$, for an interval $A=[u, v]$ of length $l=v-u$ and midpoint $\xi$, this is defined as

$$
n(\xi, l, t)=\frac{1}{2} \frac{\delta^{2} S_{A}}{\delta u \delta v}, \quad \Delta n(\xi, l, t)=\frac{1}{2} \frac{\delta^{2} \Delta S_{A}}{\delta u \delta v}
$$


where in the second equality we present the shifted entanglement density in terms of the renormalized entanglement entropy $\Delta S_{A}$. Writing $\Delta S_{A}$ in terms of $u$ and $v$ as in equation (32) and computing the derivatives gives

$$
\begin{array}{r}
l^{2} \Delta n(\xi, l, t)+\Delta S_{A}=0, \\
\lim _{l \rightarrow 0} \Delta n(\xi, l, t)=T_{00}(\xi) \lim _{l \rightarrow 0} 2 \pi \int_{-l}^{l} d x^{\prime} \frac{l^{2}-x^{\prime 2}}{2 l}=\frac{\pi}{3} T_{00}(\xi) .
\end{array}
$$

which agrees with the holographic results of [67]. Finally, we note some overlap with [8]. The author of [8] considered a gravitational theory on Rindler space and derived the change in entanglement entropy across the Rindler horizon as in equation (43) due to a metric perturbation $g_{a b} \rightarrow g_{a b}+h_{a b}$. There the modular Hamiltonian (4), was evaluated along the event horizon $\mathrm{H}$ and was shown to be equal to an operator $\hat{A}_{H}$ that measures the area of the event horizon. The crucial ingredient deriving this relation was the universal coupling $\int h_{a b} T^{a b}$ of the graviton with the energy momentum tensor, which results in a perturbative Einstein's equation that relates $T_{a b}$ to $\square h_{a b}$. Thus, the renormalized horizon entanglement entropy was found to be

$$
\delta S_{H}=\frac{\operatorname{Tr}\left(A_{H} \delta \rho_{H}\right)}{4 G}=\frac{\delta \operatorname{Area}(H)}{4 G}
$$

Even though this equation was not derived from AdS/CFT, there is an obvious parallel here with equation (55), where the minimal surface $\gamma_{A}$ is identified with the horizon $\mathrm{H}$.

\subsection{Conclusion}

In this chapter, we employed path integral methods to find a universal relation between the ground state modular Hamiltonian for an arbitrary region $A$ and the physical stress tensor. For spherical entangling surfaces in a CFT we find, as in 
[17], that the modular Hamiltonian is the integral of a local density against a local entanglement temperature. We further generalize this result to include states with conserved charges preserving conformal invariance and derive new expressions for the modular Hamiltonians in various cylindrical backgrounds in 2 dimensions. Along the way, we show that the standard results for entanglement entropy in $d=2$ dimensions that are traditionally derived from the replica trick can be obtained easily by evaluating the thermal entropy density using the entanglement temperature, and integrating over $A$. While completing this work, we became aware that the same method was used in [81] to obtain the leading area law behavior of entanglement entropy for a half space $A$ in a $d+1$-dimensional CFT and to derive the exact result for a finite interval $A$ in a $d=2 \mathrm{CFT}$ on the plane. It was also argued there that at high temperatures the entanglement entropy for theories with a mass gap $m$ can be estimated by cutting off the size of the integration region $\mathrm{A}$ at $x^{1}=\frac{1}{m}$, and indeed this gives the exact result for $d=2$. In this paper, we made the additional observation that the entanglement temperature relates the change in entanglement entropy to changes in conserved charges of the ground state via equation (50). However, we should note that the spatially varying entanglement temperature is not physical in the sense that it does not determine the expectation value of local observables such as $T_{00}$. (Indeed, $\left\langle T_{00}\right\rangle$ is a constant.) This is because the modular Hamiltonian (1) is an integral over operators that do not commute, so the reduced density matrix does not factorize. In fact, the entanglement temperature is not even conformally invariant; however equation (50) shows that in a fixed conformal frame, it gives a universal relation between the expectation value of physical charges inside a region $\mathrm{A}$ and the renormalized entanglement entropy. 


\subsection{Appendix A:Evaluating the ground state entanglement entropy from the modular Hamiltonian}

In this section we would like to point out a subtlety in evaluating the ground state entanglement entropy directly from equation (1). The discussion will also serve to provide some background for the calculation in appendix 2.9. Given the normalized reduced density, $\rho_{A}=\exp \left(-H_{A}\right) / Z_{A}$, the entanglement entropy is

$$
S_{A}=-\operatorname{Tr}_{A}\left(\rho_{A} \ln \rho_{A}\right)=\operatorname{Tr}_{A}\left(\rho_{A} H_{A}\right)+\ln Z_{A}
$$

Equation (1) implies the entanglement energy vanishes:

$$
\operatorname{Tr}\left(\rho_{A} H_{A}\right)=\int_{A} \beta(x) \operatorname{Tr}\left(\rho T_{00}\right)=\int_{A} \beta(x)\left\langle 0\left|: T_{00}:\right| 0\right\rangle=0 .
$$

In the last equality, we have normal ordered $T_{00}$ with respect to the usual Minkowski annihilation operators, so $S_{A}$ comes entirely from the "free energy" $\operatorname{term}^{13} \ln : Z_{A}:$.

However there is an alternative way to evaluate the entanglement energy by conformally mapping $\rho_{A}$ to a thermal density matrix with uniform temperature [17]. In the case of a a free scalar field in 2 spacetime dimensions and for $A=\{x>0\}$, $H_{A}=2 \pi \int_{x \geq 0} x\left[\left(\partial_{x} \phi\right)^{2}+\left(\partial_{t} \phi\right)^{2}\right] d x$ is the Rindler Hamiltonian [79]. In terms of Rindler coordinates

$$
x=e^{\xi} \cosh (\eta), \quad t=e^{\xi} \sinh (\eta),
$$

it can be written as

$$
H_{A}=2 \pi \int_{-\infty}^{\infty}\left(\partial_{\xi} \phi\right)^{2}+\left(\partial_{\eta} \phi\right)^{2} d \xi
$$

\footnotetext{
${ }^{13}$ Even though $Z_{A}$ is not an operator, we use the normal ordering symbol to highlight the fact that its value depends on normal ordering.
} 
Thus $H_{A}$ can be quantized by expanding the field in terms of plane waves in Rindler coordinates [86],

$$
\phi_{k}=\int \frac{d k}{\sqrt{4 \pi k}} b_{k} e^{i k(\xi-\eta)}+c . c ., \quad H_{A}=\int d k\left[b_{k}^{\dagger} b_{k}+(1 / 2) \delta(0)\right] k .
$$

The delta function term represents the Casimir energy and is removed by normal ordering with respect to the Rindler annihilation operator $b_{k}$. It is well known that under Rindler normal ordering, the Minkowski vacuum is thermal [86] so that

$$
\operatorname{Tr}\left[\rho_{A} H_{A}\right]=\left\langle 0\left|\vdots H_{A}:\right| 0\right\rangle=\frac{1}{12} \ln \frac{L}{\delta}
$$

where $L$ and $\delta$ are IR and UV cutoff's so that $A=[\delta, L]$. This result can be obtained by a standard computation of the average thermal energy for a free relativistic gas of massless (Rindler) particles at temperature $\frac{1}{2 \pi}$, subject to Dirichlet boundary conditions in the Rindler spatial coordinate $\xi$. Note that this differs from equation (77) due to the difference in Rindler mode vs. Minkowski mode normal ordering, which we denote by 3 and 2 dots respectively. We can also obtain the corresponding Rindler free energy by usual statistical mechanics arguments:

$$
\vdots \ln Z_{A} \vdots=\frac{1}{12} \ln \frac{L}{\delta}
$$

Adding this term to the entanglement energy (81) gives

$$
S_{A}=\frac{1}{6} \ln \frac{L}{\delta}
$$

which is consistent with the known result [60]. Since adding a normal ordering constant $a$ to $H_{A}$ corresponds to a shift $\ln Z_{A} \rightarrow \ln Z_{A}-a,(82),(81)$ and (77) implies $: \ln Z_{A}:=\frac{1}{6} \ln \frac{L}{\delta}$, which is the same as $S_{A}$ as it should be. 
The lesson here is that while $S_{A}$ is conformally invariant, neither the entanglement energy or free energy is.

To drive home this point we can derive the same result in a two dimensional Euclidean CFT, in the same spirit as [17] and [51]. The Euclidean version of the coordinate change from Minkowski to Rindler coordinates is the conformal map

$$
w=\log z, \quad z=x+i t, \quad w=\xi+i \theta,
$$

where $z$ and $w$ are the Euclideanised Minkowski/Rindler coordinates respectively and $\theta$ is the angular coordinate on the $z$ plane. The $z$ plane is mapped to a strip of length $2 \pi$ and the modular Hamiltonian on the $z$ plane is mapped to physical hamiltonian $H_{\theta}$ that evolves states along the $\theta$ direction ${ }^{14}$. For the ground state on the plane, $T(z)=0$ so that the transformation of the stress tensor ${ }^{15}$ gives $T(w)=c / 24[16]$. Integrating along $\xi$ to gives the expectation value of the Hamiltonian on the $z$ plane:

$$
\left\langle H_{\theta}\right\rangle=2 \pi \int_{w(A)} d \xi \frac{(\langle T(w)+T(\bar{w})\rangle)}{2 \pi}=\frac{c}{12} \ln \frac{L}{\delta}
$$

which is the desired result ${ }^{16}$. In the last equality we have again set $A=[\delta, L]$ on the $t=0$ slice of the $z$ plane, so that it is mapped to $w(A)=[\ln \delta, \ln (L)]$.

\subsection{Appendix B: Non-uniform excitation of 2D free scalar field}

In this appendix we provide an explicit evaluation of the entanglement energy $\delta S_{A}=\operatorname{Tr}\left(\delta \rho_{A} H_{A}\right)$ in equation (44) for a spatially non-uniform excitation of a $2 \mathrm{D}$ free

\footnotetext{
${ }^{14}$ This is the $\mathrm{d}=2$ dimensional analogue of the conformal transformation to the hyperbolic space $\mathcal{H} 40$ for the half infinite line $A$.

15 To conform with the conventions of [16] $T_{a b}$ is defined so the Hamiltonian is $H=\frac{1}{2 \pi} T_{00}$.

${ }^{16}$ One of the $2 \pi$ 's are from the length of the strip and the other from the definition of $H$ in terms of $T(w)$.
} 
scalar field and show that it is indeed equal to eq (43). First note that normal ordering is irrelevant in this case because shifting $H_{A}$ by a constant does not change the entanglement energy due to the normalization condition $\operatorname{Tr}\left(\delta \rho_{A}\right)=0$. Now following [4] we consider a particular excitation labelled by a positive Rindler momentum $k$ :

$$
d_{k}^{\dagger}|0\rangle=\int_{0}^{\infty} d p D(k, p) a_{p}^{\dagger}|0\rangle, \quad D(k, p)=(2 k \operatorname{Sinh}(\pi k))^{1 / 2} \Gamma(-i k)|p|^{i k-\frac{1}{2}}, \quad k>0
$$

where $a_{p}^{\dagger}$ are the conventional Minkowski creation operators. It is then straight forward to compute the (unnormalized) energy density by quantizing the energy density $T_{00}=\frac{1}{2}\left\{\left(\partial_{\xi} \phi\right)^{2}+\left(\partial_{\eta} \phi\right)^{2}\right\}$ in terms of Minkowski modes:

$$
\left\langle 0\left|d_{k}: T_{00}: d_{k}^{\dagger}\right| 0\right\rangle=\frac{\left(-1+e^{2 k \pi}\right) k \pi^{2} \operatorname{csch}^{2}(k \pi)}{2 \pi x^{2}}
$$

Now we compute the $\delta S_{A}$ for the half space A using the modular Hamiltonian in equation (25). Dividing by the (infinite) normalization constant $N=\left\langle 0\left|d_{k} d_{k}^{\dagger}\right| 0\right\rangle=$ $2 \pi \int_{0}^{\infty} \frac{d p}{p}$ and inserting into the equation (44) gives $^{17}$

$$
\delta S_{A}=\pi k(1+\operatorname{coth}(k \pi))
$$

Alternatively, we can evaluate $\delta S_{A}$ using equation (43) via an explicit representation of the reduced density matrix $\delta \rho_{A}$ corresponding to the state in equation (86). If we define the following reduced density matrices for the kth mode,

$$
\begin{gathered}
\rho_{0}(k)=\sum_{n_{k}=0}^{\infty} e^{-2 \pi n k}\left(1-e^{2 \pi k}\right)\left|n_{k}\right\rangle\left\langle n_{k}\right|, \\
\rho_{1}(k)=\sum_{n_{k}=0}^{\infty}\left(4 n_{k} \sinh ^{2}(\pi k)\right)\left|n_{k}\right\rangle\left\langle n_{k}\right|,
\end{gathered}
$$

\footnotetext{
${ }^{17}$ The logarithmically divergent integral over $\mathrm{x}$ is cancelled by the normalization $N$.
} 
where $\left|n_{k}\right\rangle$ denotes the occupational number basis for the Rindler particles, then results of [4] imply that $\delta \rho_{A}=\rho_{1}(k) \prod_{l \neq k} \rho_{0}(l)-\prod_{l} \rho_{0}(l)$. Inserting this into (44) and evaluating the trace using the Rindler Hamiltonian (80) gives

$$
\begin{aligned}
\delta S_{A} & =2 \pi \sum_{n \geq 1} e^{-2 \pi n k}\left(4 n \sinh ^{2}(\pi k)-\left(1-e^{-2 \pi k}\right)\left\langle n_{k}\left|\vdots H_{A} \vdots\right| n_{k}\right\rangle\right. \\
& =2 \pi \sum_{n \geq 1} e^{-2 \pi n k}\left(4 n \sinh ^{2}(\pi k)-\left(1-e^{-2 \pi k}\right)\right) n k \\
& =\pi k(1+\operatorname{coth}(\pi k)),
\end{aligned}
$$

which is the same result as equation (18). 


\section{Chapter III}

\section{Entanglement branes in a two dimensional string theory}

\subsection{Introduction}

As reviewed in chapter 1, Entanglement in string theory has been mostly studied using the replica trick $[78,80,21,20]$. To calculate the entropy via the replica trick one considers the Euclidean path integral $Z(\beta)$ on a spacetime where the angular coordinate $\phi$ is identified with period $\beta$. When $\beta \neq 2 \pi$, this spacetime is singular, containing a planar conical defect of angle $\beta$. The replica trick then gives the entropy as

$$
S=\left.\left(1-\beta \partial_{\beta}\right) \log Z\right|_{\beta=2 \pi}
$$

Ref. [80] showed how the entropy calculation could be organized into contributions from different closed string worldsheets as shown in figure 3.2. It was argued that the genus-zero worldsheets that intersect the conical singularity, one of which is shown in figure 3.1, give the classical Bekenstein-Hawking term, and the torus diagrams give its one-loop correction. These different diagrams can be foliated by an angular coordinate going around the entangling surface, and describe propagation of both closed and open strings, where the open string endpoints are anchored to the entangling surface. 

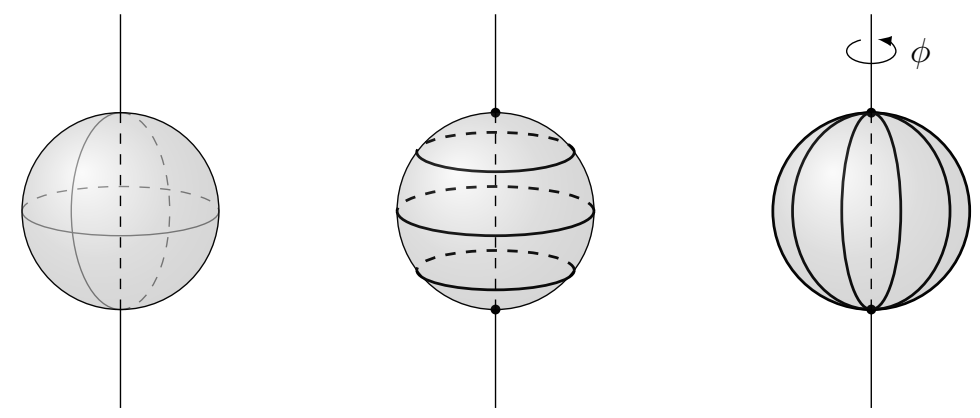

Figure 3.1:

The diagrams that dominate in the calculation of entanglement entropy are sphere diagrams such as the one depicted on the left. Sliced transverse to the entangling surface, as in the middle figure, this diagram describes a closed string emitted from a point on the entangling surface and then reabsorbed. Sliced in angular time $\phi$, the sphere diagram is a one loop open string diagram with the endpoints fixed on the entangling surface as depicted on the right.

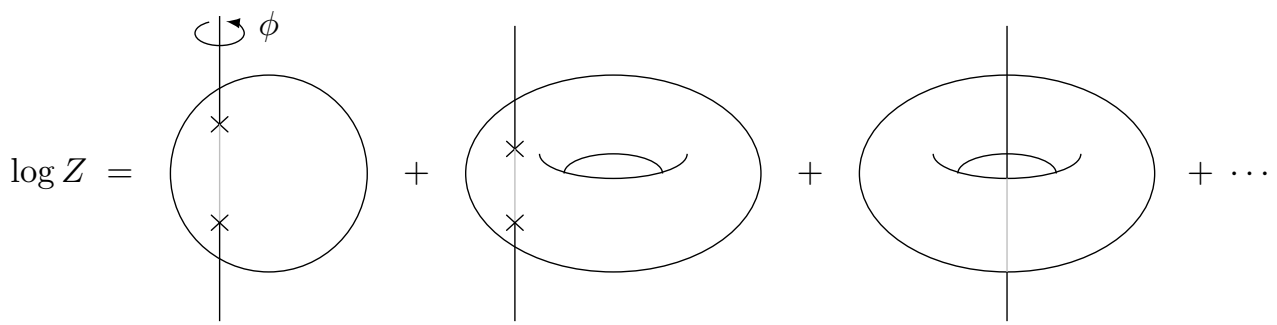

Figure 3.2:

In string theory, $\log Z(\beta)$ is a sum of connected string diagrams embedded in a Euclidean spacetime with a conical defect of angle $\beta$ at the entangling surface. Only closed string diagrams that intersect or encircle the entangling surface, such as those depicted above, contribute to the entanglement entropy. 
While the replica trick calculation is very efficient, it is somewhat opaque in that it does not provide a canonical description of the entropy. This raises an important question: which states are being counted in this calculation? The replica calculation is further complicated by the fact that it relies on the path integral in the background of a conical defect, for which one would have to define string theory off-shell.

A closely related issue arises in field theory, and it concerns the way that certain fields couple to a conical singularity in spacetime. For fields with nontrivial coupling to curvature the path integral on a cone contains in "contact terms", which are explicit interactions between the curvature and the dynamical fields. These contact terms arise for scalar fields with nonminimal coupling [61, 75] and for gauge fields [59]. String theory contains fields of all spins, and hence an infinite tower of such contact terms [50]. In theories with massless fields of spin $3 / 2$ or spin 2 the situation is even worse: the theory cannot be consistently defined on manifolds such as the conical defect that are not solutions of the Einstein equation [39].

The presence of contact terms obscures the interpretation of the entropy as calculated via the replica trick (1). Does the replica formula still calculate the von Neumann entropy of a reduced density matrix, or is there a distinct non-entanglement contribution from contact interactions? Recently it has been understood that the contact terms in abelian gauge theory do correspond to a counting of states, but the entanglement entropy has to be suitably interpreted in a gauge theory. Since the physical Hilbert space does not factorize, one must consider an extended Hilbert space containing degrees of freedom associated with the boundary surface [25]. In the case of electrodynamics the entropy of these edge modes have been shown to give rise to the contact terms in the entropy $[30,31]$. The inclusion of the edge modes resolves 
the longstanding puzzle of the interpretation of the contact term, and are necessary to agreement between the logarithmic divergence and the central charge. ${ }^{1}$

The decomposition of the Hilbert space in terms of edge modes is best understood for Yang-Mills theory in two spacetime dimensions [27]. Two-dimensional YangMills theory has no local degrees of freedom and so one avoids all issues related to ultraviolet divergences. The theory is almost topological, and can be exactly solved by topological quantum field theory methods. The partition function of a closed oriented manifold $M$ depends only on its topology (which is encoded in the Euler characteristic $\chi=2-2 G$, where $G$ is the genus) and its total area $A$. The partition function can be written exactly as [18]:

$$
Z=\sum_{R}(\operatorname{dim} R)^{\chi} e^{-\frac{\lambda A}{2 N} C_{2}(R)}
$$

where $\lambda=g_{\mathrm{YM}}^{2} N$ is the (dimensionful) 't Hooft coupling. Here $R$ runs over all irreducible representations of the gauge group, which we will take to be $\mathrm{U}(N)$, with $\operatorname{dim}(R)$ the dimension of each representation and $C_{2}(R)$ the quadratic Casimir.

Entanglement entropy for general regions can be obtained from applying the replica formula (1) to the partition function (2) [26, 44]. To make sense of this formula and to see which states the entanglement entropy counts, we have to understand how to decompose the Hilbert space into regions of space. In $1+1$ dimensions, Hilbert spaces are associated to 1-dimensional manifolds, which may be circles or intervals. On a circle, the Hilbert space is the space of square-integrable class functions on the group, i.e. those functions $\psi: G \rightarrow \mathbb{C}$ satisfying $\psi(U)=\psi\left(g^{-1} U g\right)$. On an interval the Hilbert space is the space of square-integrable functions on the gauge group, with no restriction to class functions. This space carries two unitary actions of $G$, corresponding to left and right group multiplication, and which act at the left

\footnotetext{
${ }^{1}$ The apparent negative sign of the contact term found in ref. [59] and emphasized in ref. [29] is a red herring; the leading divergence depends on the regularization scheme and may have either sign [31].
} 
and right endpoints of the interval, respectively. Two intervals can be glued together at a common endpoint using the entangling product [28]; we take the ordinary tensor product of Hilbert spaces and quotient by the simultaneous action of right multiplication on the leftmost interval and left multiplication on the rightmost interval. This allows us to combine two intervals into the Hilbert space of a larger interval, or to glue both endpoints of an interval together, giving the Hilbert space of a circle. Using this decomposition, we can embed the state of any region into the tensor product of the subregions and the resulting entanglement entropy reproduces the result of the replica trick calculation [26].

How do we use this insight from Yang-Mills theory to understand entanglement entropy in string theory? The key observation we will exploit, due to Gross and Taylor is that in the large- $N$ limit, Yang-Mills theory is a string theory $[45,47]$. The partition function (2) can be expressed as a sum over maps from a two-dimensional worldsheet into the spacetime manifold $M$, weighted by the Nambu-Goto action. The worldsheet can have certain prescribed singularities that represent interactions of the strings. $^{2}$ There are additional singularities that must appear on manifolds with $\chi \neq 0$ such as the sphere, which Gross and Taylor called $\Omega$-points. The $\Omega$-points are unlike the other singularities: they are not integrated over, and their total number is fixed by the Euler characteristic of the manifold. While their existence is demanded from the evaluation of the Yang-Mills partition function, the reason for the $\Omega$-point singularities from the perspective of the string theory path integral is somewhat obscure.

In order to import our understanding of entanglement in Yang-Mills theory into the Gross-Taylor description, we have to describe the theory canonically in terms of a Hamiltonian operator acting on a Hilbert space. Baez and Taylor showed how to

\footnotetext{
${ }^{2}$ One can give a string theory interpretation of two-dimensional Yang-Mills theory for gauge groups $\mathrm{U}(N), \mathrm{SU}(N)$ and even $\mathrm{SO}(N)$ and $\mathrm{Sp}(N)$ [71]. These theories differ in the orientability of the worldsheet, and in the types of singularities that can appear. Here we will focus on a subsector of the $\mathrm{U}(N)$ theory that leads to the simplest string description, but we expect the broad features to generalize.
} 
describe the Hilbert space of a circle and its time evolution along a cylinder in the Hamiltonian language as a string field theory [2]. We review this picture in section 3.2. The states in this Hilbert space are labeled by collections of closed strings winding around the spatial circle, and the dynamics consists of local interactions that cut and reglue strings at the same point. The sphere partition function can also be interpreted as a closed string amplitude, by foliating the worldsheet as in the middle diagram of figure 3.1 .

In order to describe entanglement in the string theory picture, we must also describe the Hilbert space of an interval, and the entangling product that connects two intervals. In section 3.3 we show how to describe the Hilbert space of an interval in terms of a canonical theory of open strings. Each open string carries a pair of Chan-Paton indices $i, j=1, \ldots, N$ associated with its two endpoints. The entangling product can be seen as a relation between the closed string and open string Hilbert spaces:

$$
\mathcal{H}_{\text {closed }} \subset \mathcal{H}_{\text {open }} \otimes \mathcal{H}_{\text {open }}
$$

This says that any closed string may be cut into open strings, but that not every collection of open strings can be reassembled into closed strings; this requires a specific matching of the Chan-Paton indices, which leads to a special entanglement structure of the closed string states. We also derive the modular Hamiltonian for an interval on the sphere, and show that it corresponds to a Nambu-Goto term plus local interactions of the open strings.

The key point is that in the open string description, the partition function contains a sum over Chan-Paton indices associated with the string endpoints. This results in an extra statistical weight associated to the $N^{2}$ possible values of the two Chan-Paton indices on each open string: this factor is directly responsible for the leading-order $N^{2}$ scaling of the entanglement entropy. We find that the statistical weight associated with the open string edge modes is encoded in the sum over worldsheets by the $\Omega$ - 
point singularity. We argue that the two $\Omega$-points appearing on the sphere should be identified with the entangling surface, on which open strings are allowed to end. This is similar to the way that open strings can end on a D-brane, so we call the resulting objects E-branes. The E-brane encodes how the edge degrees of freedom appear at the level of the partition function, and also explains the appearance of Gross and Taylor's $\Omega$-point. This is the central result of our paper.

In the large- $N$ limit the theory is described by two distinct chiral sectors, and in section 3.3 we focus for simplicity on the theory restricted to a single sector. In section 3.4 we describe the additional features the theory acquires when both sectors are included. In this case the path integral contains worldsheets of two distinct orientations, and a new class of singularities - orientation-reversing tubes - can appear at the $\Omega$-points. We show that these singularities, like the other aspects of the $\Omega$-points, are purely kinematical and simply enforce the unitarity condition $U^{-1}=U^{\dagger}$

We conclude in 3.5 with some areas of future work, as well as a discussion on entanglement in string theory in higher dimensions.

\subsection{Two-dimensional Yang-Mills theory as a closed string theory}

Here we review the description of two-dimensional Yang-Mills theory as a theory of closed strings. This will serve to establish some basic definitions for use later on, and motivates our generalization to open strings. See ref. [3] for an introduction, or ref. [18] for a more comprehensive review. 


\subsubsection{The closed string Hilbert space}

We consider two-dimensional Yang-Mills theory with gauge group $G=\mathrm{U}(N)$, with Euclidean action

$$
I=\frac{1}{4 g_{\mathrm{YM}}^{2}} \int_{M} \sqrt{g} \operatorname{tr}\left[F^{\mu \nu} F_{\mu \nu}\right] .
$$

We first consider the Hamiltonian formulation of the theory where space is a circle of circumference $L$. The theory has no local degrees of freedom, and the gaugeinvariant variables can be constructed from the holonomy $U$ around the circle, and the nonabelian electric field $E^{a}$ :

$$
U=\mathcal{P} \exp \left(i \int_{0}^{L} d x A_{x}(x)\right) \in G, \quad E^{a}(x)=-i g_{\mathrm{YM}}^{2} \frac{\delta}{\delta A_{a}(x)} \in \mathfrak{g}
$$

The Hilbert space of states on the circle is the space of square-integrable class functions on the group manifold. These are functions $\psi$ of the holonomy $U$ which are square integrable in the Haar measure, and invariant under the gauge transformation: $\psi(U)=\psi\left(g U g^{-1}\right)$.

The Hamiltonian is proportional to the square of the electric field (there are no magnetic fields in $1+1$ dimensions) which can be written explicitly as a second-order differential operator (the Laplacian) on the group manifold:

$$
\begin{aligned}
H & =\frac{1}{2 g_{\mathrm{YM}}^{2}} \int_{0}^{L} d x E^{a}(x) E^{a}(x) \\
& =-\frac{g_{\mathrm{YM}}^{2}}{2} \int_{0}^{L} d x \frac{\delta}{\delta A_{a}(x)} \frac{\delta}{\delta A_{a}(x)} \\
& =\frac{\lambda L}{2}\left(\sum_{i j} U_{i j} \frac{\partial}{\partial U_{i j}}+\frac{1}{N} \sum_{i j k l} U_{i k} U_{j l} \frac{\partial}{\partial U_{j k}} \frac{\partial}{\partial U_{i l}}\right)
\end{aligned}
$$

where we have used the 't Hooft coupling $\lambda=g_{\mathrm{YM}}^{2} N$. 
A natural basis of the Hilbert space is given by states $|R\rangle$ whose wave functions are the irreducible characters of the group:

$$
\langle U \mid R\rangle=\chi_{R}(U)
$$

The virtue of this basis is that it diagonalizes the Hamiltonian (8). The corresponding eigenvalues are proportional to the quadratic Casimir of each representation $H|R\rangle=$ $\frac{\lambda L}{2} C_{2}(R)|R\rangle$.

To describe this theory as a string theory, we introduce a basis of states describing closed strings [2]. Let $\sigma \in S_{n}$ be a permutation with $n_{l}$ cycles of length $l$. We define a state $|\sigma\rangle$ with $n_{l}$ closed strings winding $l$ times around the circle by

$$
\langle U \mid \sigma\rangle=\prod_{l=1}^{\infty}\left(\operatorname{Tr} U^{l}\right)^{n_{l}}, \quad \quad \sum_{l} n_{l}=n .
$$

Consistent with closed string indistinguishability, these wave functions depend only on $\left\{n_{l}\right\}$, which specifies the conjugacy class of $\sigma .^{3}$

The two sets of states are related by the Frobenius relations:

$$
|R\rangle=\sum_{\sigma \in S_{n}} \frac{\chi_{R}(\sigma)}{n !}|\sigma\rangle
$$

Here we have used the fact that the irreducible representations of $\mathrm{U}(N)$ and of the permutation group $S_{n}$ can be represented by Young diagrams: $n$ is the total number of boxes in the Young diagram associated to $R$, and $\chi_{R}(\sigma)$ is the associated character of the permutation group. If we number the matrices $U$ in the product (8) and represent each as an open string wrapped around the spatial circle, then the permutation $\sigma$

\footnotetext{
${ }^{3}$ In the full $\mathrm{U}(N)$ theory, the string states (8) span only a subspace of $\mathcal{H}$. These can be extended to a complete set by including wave functions which contain an additional factor of $\operatorname{det}(U)^{m}$. In this paper we will restrict to the $m=0$ sector, for which the string states (8) give a complete description. The quantum number $m$ is related to the $\mathrm{U}(1)$ charge $Q$ labeling the total number of boxes in the Young diagram of $R$ by $Q=m N+n$.
} 


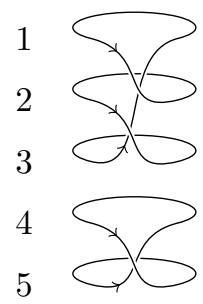

Figure 3.3:

The closed string configuration corresponding to the permutation $\sigma$ : $1\rangle 2,2\rangle 3,3\rangle 1,4\rangle 5,5\rangle 4$. The cycle lengths $(3,2)$ correspond to the winding numbers of the closed strings.

specifies the index contractions that glues the open string endpoints into a closed string configuration as illustrated in figure 3.3. Applied to a closed string state $|\sigma\rangle$, the leading term of the Hamiltonian (8) counts the total winding number $n$, while the subleading term is a sum of interactions that locally cut and reconnect the strings. In terms of permutations, each of these interactions act as transpositions $p \in T_{2}$ taking $|\sigma\rangle \rightarrow|p \sigma\rangle$ which changes the individual winding numbers, but not the total winding number. We can decompose the Hamiltonian into a free and interacting part:

$$
\begin{aligned}
H & =H_{0}+2 H_{1}, \\
H_{0}|\sigma\rangle & =\frac{\lambda L}{2} n|\sigma\rangle, \\
H_{1}|\sigma\rangle & =\frac{\lambda L}{2 N} \sum_{p \in T_{2}}|p \sigma\rangle .
\end{aligned}
$$

Since $H_{1}$ contains an explicit factor of $1 / N$, the interaction can be treated as subleading in the large $N$ limit. This is the limit in which the string theory becomes weakly coupled.

We now consider how to describe the Hilbert space of the theory in the large $N$ limit. The basis states $|R\rangle$ are labelled by representations, but we must define how to fix a representation as $N \rightarrow \infty$. Naively one could fix the Young diagram for $|R\rangle$, thus fixing the right hand side of the Frobenius relation (9), which remains well-defined in the large- $N$ limit. However, this keeps only states whose Young diagrams have a 
finite number of boxes, essentially truncating the theory in half: for example, this procedure would exclude the antifundamental representation, whose Young diagram is a column of height $N-1$. The full Hilbert space at large $N$ can be identified as a subspace of the tensor product

$$
\lim _{N \rightarrow \infty} \mathcal{H}_{\mathrm{U}(N)} \subset \mathcal{H}^{+} \otimes \mathcal{H}^{-}
$$

where $\mathcal{H}^{ \pm}$are spanned by representations built out of finite numbers of fundamentals or antifundamentals respectively. These two chiral sectors consist of closed strings winding in opposite orientations: $\mathcal{H}^{+}$is spanned by the closed string basis $(8)$ and $\mathcal{H}^{-}$ is spanned by a similar basis with $U$ replaced by $U^{\dagger}$. This is the correct Hilbert space in the sense that the asymptotic $\frac{1}{N}$ expansion of the Yang-Mills partition function (2) requires a sum over both chiral sectors. To make $\mathcal{H}^{+}$explicit as a multi-string Fock space, we define the vacuum and the creation operator for a string winding $l$ times:

$$
\begin{aligned}
\langle U \mid 0\rangle & =1 \\
\left\langle U\left|a_{l}^{\dagger}\right| 0\right\rangle & =\operatorname{Tr}\left(U^{l}\right) \\
|\sigma\rangle & =\prod_{l}\left(a_{l}^{\dagger}\right)^{n_{l}}|0\rangle
\end{aligned}
$$

$a_{l}^{\dagger}$ acts by multiplication by $S_{l}=\operatorname{Tr}\left(U^{l}\right)$. It can be shown that the corresponding adjoint operator is $a_{l}=l \frac{\partial}{\partial S_{l}}$, which satisfies the commutation relations and normalizations:

$$
\begin{aligned}
{\left[a_{l}, a_{l^{\prime}}^{\dagger}\right] } & =l \delta_{l l^{\prime}} \\
\langle\sigma \mid \sigma\rangle & =\prod_{l} l^{n_{l}} n_{l} !
\end{aligned}
$$

Note that the normalization of $a_{l}$ differs from the usual quantum-mechanical convention. The normalization of $|\sigma\rangle$ counts the number of permutations commuting with 
$\sigma$, which coincides with the order of the stabilizer subgroup, $\langle\sigma \mid \sigma\rangle=\left|C_{\sigma}\right|$, where

$$
C_{\sigma}:=\left\{\tau: \sigma=\tau \sigma \tau^{-1}\right\}
$$

Let $T_{\sigma}$ denote the orbit of the permutation $\sigma$ under conjugation,

$$
T_{\sigma}:=\left\{\tau \sigma \tau^{-1}: \tau \in S_{n}\right\}
$$

The orbit-stabilizer theorem states that the size of the orbit times the size of the stabilizer equals the order of the group:

$$
\left|T_{\sigma}\right|\left|C_{\sigma}\right|=\left|S_{n}\right|=n !
$$

Accounting for this normalization and closed string indistinguishability, we can write a resolution of identity in the $|\sigma\rangle$ basis,

$$
1=\sum_{n} \sum_{\sigma \in S_{n}} \frac{|\sigma\rangle\langle\sigma|}{\left|T_{\sigma}\right|\left|C_{\sigma}\right|}=\sum_{n} \frac{1}{n !} \sum_{\sigma \in S_{n}}|\sigma\rangle\langle\sigma|
$$

where in the first equality we have divided by $\left|T_{\sigma}\right|$ because all elements in the same conjugacy class represent the same closed string state, and divided by the normalization factor $\left|C_{\sigma}\right|$. In the second equality, we applied the orbit-stabilizer theorem.

In terms of these closed string creation and annihilation operators, the Hamilto$\operatorname{nian}(10)$ is $[66]$

$$
H=\frac{\lambda L}{2}\left[\sum_{k=1}^{\infty} a_{k}^{\dagger} a_{k}+\frac{1}{N} \sum_{k, l=1}^{\infty}\left(a_{k+l}^{\dagger} a_{k} a_{l}+a_{k}^{\dagger} a_{l}^{\dagger} a_{k+l}\right)\right]
$$

This Hamiltonian defines the closed string field theory dual to the chiral sector of two-dimensional Yang-Mills. The first term is the free term, which is proportional to the length of the interval times total winding number of the strings. This is the string 
tension, which is proportional to the total length of strings. The second term is an interaction in which closed strings interact by splitting and joining via a cubic vertex. This interaction corresponds to two strings of winding numbers $k$ and $l$ merging into a string of winding number $k+l$, and the reverse process. This interaction preserves the total winding number, so it commutes with the free term.

The Hamiltonian (18) does not capture the full theory, since the operators $a_{k}^{\dagger}$ only creates states that can be obtained by acting on the vacuum state with multiplication by $\operatorname{tr}\left(U^{k}\right)$, which are all holomorphic wavefunctions. To get the full $\mathrm{U}(N)$ theory we would have to include a sector of antiholomorphic wavefunctions. These can be thought of as strings winding in the opposite direction, and will be developed further in section 3.4 .

Finally, at finite $N$, there are Mandelstam identities that force us to identify certain string states; for example, in the U(1) theory, $a_{2}^{\dagger}|0\rangle=a_{1}^{\dagger} a_{1}^{\dagger}|0\rangle$. These can be implemented by a projection operator as shown in ref. [2]. The effect of this projection is nonperturbative in the $1 / N$ expansion, so we will neglect it in the following.

\subsubsection{Torus partition function}

To illustrate how the worldsheet expansion of two-dimensional Yang-Mills theory emerges, we now perform a perturbative expansion of the Yang-Mills partition function $Z_{T^{2}}$ on a torus and construct the corresponding closed string Feynman diagrams. We will see that the Hamiltonian evolution in Euclidean time $\beta$ of a multiply-wound closed string state traces out a multi-sheeted Riemann surface on which singularities appear whenever the strings interact by joining or breaking apart. Since the total winding number is conserved, the Hilbert space naturally divides into sectors labelled by $n$, which we denote $\mathcal{H}_{n}$, and the partition function decomposes as a sum:

$$
Z_{T^{2}}=\operatorname{tr}\left(e^{-\beta H}\right)=\sum_{n} e^{-\frac{\lambda n A}{2}} \operatorname{tr}_{\mathcal{H}_{n}}\left(e^{-\beta H_{1}}\right)
$$


We recognize the leading term as the Nambu-Goto action of a string world sheet wrapping $n$ times around the torus of area $A=L \beta$.

To calculate the effect of the interaction term, we expand the exponential

$$
\begin{aligned}
\operatorname{tr}_{\mathcal{H}_{n}}\left(e^{-\beta H_{1}}\right) & =\sum_{i} \frac{(-1)^{i}}{i !}\left(\frac{\lambda A}{N}\right)^{i} \operatorname{tr}_{\mathcal{H}_{n}}\left(H_{1}^{i}\right) \\
& =\sum_{i} \frac{(-1)^{i}}{i !}\left(\frac{\lambda A}{N}\right)^{i} \sum_{\sigma \in S_{n}} \frac{1}{n !} \sum_{p_{1}, \ldots, p_{i} \in T_{2}}\left\langle\sigma\left|p_{1} \cdots p_{i}\right| \sigma\right\rangle .
\end{aligned}
$$

We recognize this as a sum over $i$ interaction points, with a factor of $1 / i$ ! indicating the indistinguishability of the interactions. ${ }^{4}$ The factor of $(1 / N)$ is the string coupling associated with each interaction and $\lambda A$ is a modulus factor, which comes from integrating over all possible locations for the interaction. We can further expand this sum, by noting that the matrix element $\left\langle\sigma\left|p_{1} \cdots p_{i}\right| \sigma\right\rangle$ is nonzero precisely when there exists $\tau \in S_{n}$ such that

$$
p \sigma=\tau \sigma \tau^{-1}
$$

where we have defined $p=p_{1} \cdots p_{i}$. Thus we can introduce an explicit sum over $\tau$, at the expense of dividing by the order of the stabilizer group, and rewrite the matrix element in (20) as

$$
\langle\sigma|p| \sigma\rangle=\sum_{\tau \in S_{n}} \delta\left(p \sigma, \tau \sigma \tau^{-1}\right)
$$

Above, the normalization of $|\sigma\rangle$ has canceled the division by $\left|C_{\sigma}\right|$. Using (20) and (22), the torus partition function is:

$$
Z_{T^{2}}=\sum_{n} \frac{e^{\frac{-n \lambda A}{2}}}{n !} \sum_{i} \frac{(-1)^{i}}{i !}\left(\frac{\lambda A}{N}\right)^{i} \sum_{p_{1} \ldots p_{i} \in T_{2}} \sum_{\sigma, \tau \in S_{n}} \delta\left(p \sigma, \tau \sigma \tau^{-1}\right)
$$

\footnotetext{
${ }^{4}$ We are not aware of any physical interpretation for the factor of $(-1)^{i}$, but it is vaguely suggestive of a fermionic nature of the interaction points.
} 


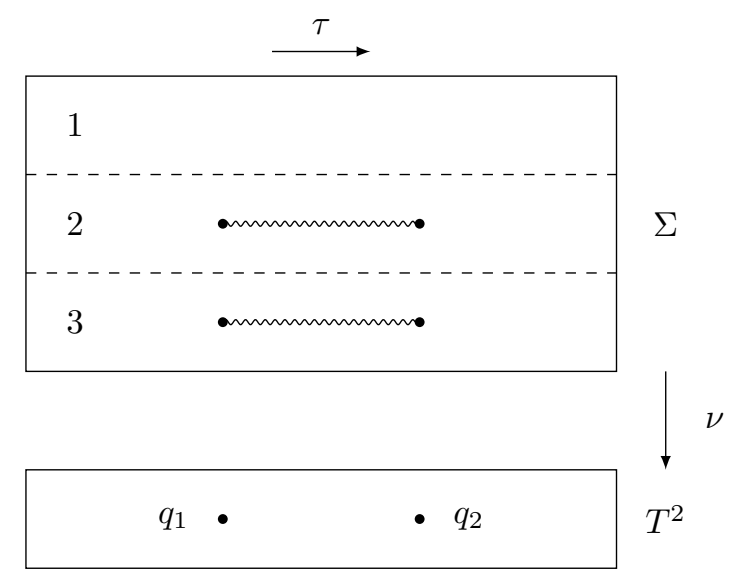

Figure 3.4:

A 3-sheeted covering map of the torus $T^{2}$ with two interaction branch points $q_{1}, q_{2}$. The covering map $\nu$ is defined here by vertical projection. A counterclockwise loop encircling the target space branch point $q_{1}$ lifts to a permutation $p_{1}: 1 \rightarrow 1,2 \rightarrow 3,3 \rightarrow 2$. A vertical time slicing of $\Sigma$ shows an initial closed string breaking into two and then joining back together again.

The expression (23) was obtained by Gross and Taylor [47], who interpreted it as a sum over branched coverings of the torus $T^{2}$. An $n$-sheeted covering $\left.\nu: \Sigma\right\rangle T^{2}$ with $i$ branch points $q_{1}, \ldots, q_{i} \in T^{2}$ is uniquely specified by a homomorphism

$$
\left.H_{\nu}: \pi_{1}\left(T^{2} \backslash\left\{q_{1}, \ldots, q_{i}\right\}\right)\right\rangle S_{n} .
$$

This homomorphism describes how the $n$ sheets are permuted as we follow loops on $\Sigma$ obtained by lifting non-contractible curves on the punctured torus $T^{2} \backslash\left\{q_{1}, \ldots, q_{i}\right\}$. In particular each set of permutations $p_{1}, \cdots p_{i}, \tau, \sigma \in S_{n}$ satisfying the delta function constraint in (23) specifies such a homomorphism by describing how the $n$ sheets are shuffled when we encircle the branch points $q_{1}, \cdots q_{i}$ and the two basis cycles of the torus.

As alluded previously, we interpret the covering space $\Sigma$ as a closed string worldsheet wrapping the torus $n$ times (see figure 3.4) . Choosing fixed time slices running 
perpendicular to the branch cuts on $\Sigma$ shows that the branch points corresponds to interactions where closed strings break apart and join together. The $N$ dependence of the expression (23) can be understood from the Riemann-Hurwitz formula, which relates the Euler characteristic of the covering space $\chi(\Sigma)$ to the Euler characteristic of the target space $\chi(M)$ in the presence of $i$ elementary branch points.

$$
\chi(\Sigma)=n \chi(M)-i
$$

Choosing $M=T^{2}$ gives $\chi(\Sigma)=-i$, so the $N$ dependence in (23) is $N^{\chi(\Sigma)}$, giving the correct exponent for the string coupling. Finally, the $\frac{1}{n !}$ factor accounts for the redundancy from summing over homomorphisms $H_{\nu}$ that differ only by the relabelling of the $n$ sheets. The cancellation is not exact, because there are relabelling of the $n$

sheets that fix $\nu$, leading to a symmetry factor that we denote by $\frac{1}{\left|S_{\nu}\right|}$. Thus we are led to a closed string partition function which can be expressed in a compact form $[47]:$

$$
Z=\int_{C(M)} d \nu\left(\frac{1}{N}\right)^{2 g-2} \frac{(-1)^{i}}{\left|S_{\nu}\right|} e^{\frac{-n \lambda A}{2}}
$$

Here $C(M)$ denotes a set of covering maps of $M$.

\subsubsection{Sphere partition function}

The partition function on a sphere is obtained by gluing together the path integrals on a twice-punctured sphere and two infinitesimal disks. In the string picture, we can view the gluing as a closed string evolution between initial and final states inserted at the two punctures, known as $\Omega$-points [46]:

$$
Z_{S^{2}}^{+}=\left\langle\Omega\left|e^{-\beta H}\right| \Omega\right\rangle
$$


The superscript "+" denotes that we are considering only a single chiral sector, c.f. section 3.2.1. The state $|\Omega\rangle$, which is defined by the path integral over an infinitesimal disk, can be written in a number of different ways.

In the holonomy representation the state $|\Omega\rangle$ is given by

$$
\langle U \mid \Omega\rangle=\delta(U)
$$

where here $\delta$ denotes the Dirac delta function in the Haar measure supported on the identity element. This expresses the fact that as a circle contracts its holonomy becomes trivial. ${ }^{5}$

This state can be written equivalently in the representation basis as

$$
|\Omega\rangle=\sum_{R} \operatorname{dim} R|R\rangle
$$

The factor of $\operatorname{dim} R$ has an important effect on the entanglement entropy: it leads to an additional $\log \operatorname{dim} R$ factor in the entropy that represents a sum over degenerate states in each representation of the gauge group [27].

In the string basis, we can similarly write ${ }^{6}$

$$
|\Omega\rangle=\sum_{n} \frac{1}{n !} \sum_{\sigma \in S_{n}} N^{K_{\sigma}}|\sigma\rangle
$$

where $K_{\sigma}$ denotes the number of cycles in the permutation $\sigma$. The amplitude $\langle\sigma \mid \Omega\rangle=$ $N^{K_{\sigma}}$ plays a similar role to the $\operatorname{dim} R$ factor in the Yang-Mills description: it leads

\footnotetext{
${ }^{5}$ Note that unlike the case of a conformal theory, the state generated by the path integral on a small disk with no insertions is not the vacuum. The vacuum is a constant wavefunction in the $U$ basis, and corresponds to the path integral over a large disk. Two-dimensional Yang-Mills theory is not scale-invariant, so a small disk is not equivalent to a large one.

${ }^{6}$ To derive (31) we used the fact that for $R$ corresponding to a Young diagram with $n$ boxes
}

$$
\operatorname{dim} R=\sum_{\sigma \in S_{n}} \frac{\chi_{R}(\sigma)}{n !} N^{K_{\sigma}}
$$

as can be seen by setting $U=1$ in the Frobenius relation (9). 


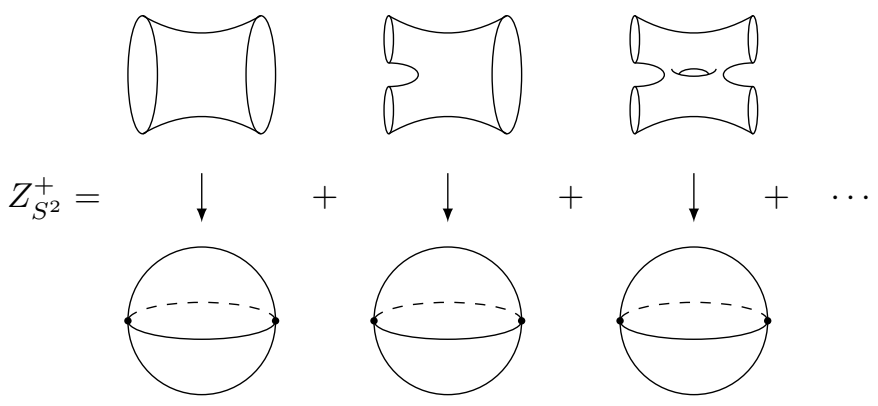

Figure 3.5:

$Z_{S^{2}}^{+}$can be expressed as a sum over worldsheets with holes corresponding to closed strings that wind around the $\Omega$-points on $S^{2}$.

to a counting of degenerate states that contribute to the entropy, as we will show in section 3.3 .

Using the state (31) and the closed string Hamiltonian (10), we can then write the sphere partition function as

$$
\begin{aligned}
Z_{S^{2}}^{+} & =\left\langle\Omega\left|e^{-\beta H}\right| \Omega\right\rangle \\
& =\sum_{n} \frac{e^{-\frac{n \lambda A}{2}}}{n !} \sum_{i} \frac{(-1)^{i}}{i !}\left(\frac{\lambda A}{N}\right)^{i} \sum_{\sigma \in S_{n}} \sum_{p_{1}, \cdots, p_{i} \in T_{2}} N^{K_{\sigma}} N^{K_{p_{1} \cdots p_{i} \sigma}},
\end{aligned}
$$

We interpret the sum over $\sigma \in S_{n}$ as a sum over world sheets $\Sigma$ on which an initial state of $K_{\sigma}$ closed strings evolves into a final state of $K_{p_{1} \cdots p_{i} \sigma}$ closed strings via $i$ interactions (see figure 3.5). Such a world sheet wraps the punctured sphere $n$ times with $i$ elementary branch points $p_{1}, \ldots, p_{i}$. The closed strings in the external states correspond to infinitesimal holes on the world sheet that wind around the two $\Omega$-points according to the cycle lengths of $\sigma$, and $p_{1} \cdots p_{i} \sigma$. Closing up the holes leads to a covering map for which the $\Omega$-point is a multiple branch point singularity labeled by the permutation $\sigma$ or $p_{1}, \cdots p_{i} \sigma$ (see figure 3.5). Thus, we can once again appeal to (25) to determine the Euler characteristic

$$
\chi(\Sigma)=-i+K_{\sigma}+K_{p_{1} \cdots p_{i} \sigma}
$$




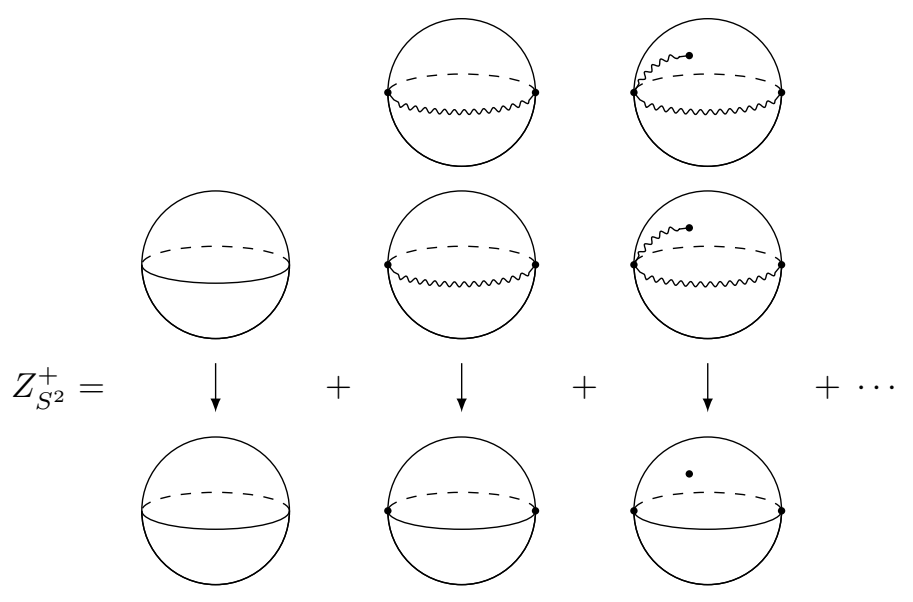

Figure 3.6: By closing up the boundaries of the worldsheet into branch points and introducing appropriate branch cuts, we can present the worldsheet as a covering space of the sphere, with covering map represented by vertical projection. The first is a single covering with no interaction and $\Omega$-point singularities. The second is a double cover of the sphere with two $\Omega$ points. The third term corresponds to the "pair of pants" diagram, now presented as a double cover with an interaction branch point inserted, which is connected to an $\Omega$-point via a branch cut.

consistent with the power of the string coupling in (32). As before, the division by $n$ ! accounts for the partial redundancy due to relabelings of the $n$ sheets, and leads to the correct symmetry factor for each diagram.

Thus we conclude that the closed string expansion (26) continues to hold on the sphere, provided that the space of covering maps is extended to allow for two $\Omega$-points, which are fixed point of $M$ at which multiple branch points can appear. While the presence of such branch point singularities was derived in [46], we have introduced a new interpretation of them as sources that emit and absorb closed strings with coupling $N=\frac{1}{g_{\text {string }}}$ per closed string. The state $|\Omega\rangle$ is therefore analogous to a Dbrane boundary state, which has the same coupling to closed strings. We will call it an entanglement brane or E-brane. The presence of brane-like objects suggests the presence of open strings which would couple to the E-brane. In the next section we will pursue the open string description of the $\Omega$-points and reinterpret them as entangling surfaces in string theory. 


\subsection{Angular quantization on the sphere and open strings}

In the preceding section we have expressed the sphere partition function in terms of closed strings propagating between two E-brane boundary states $|\Omega\rangle$. This sphere partition function is the key ingredient that enters in calculating the entanglement entropy in two-dimensional de Sitter space. In order to see which states are being counted by this entanglement calculation, we have to express the partition function as a trace of the form $\operatorname{tr} e^{-\beta H_{V}}$ — the key is to identify the appropriate Hilbert space and modular Hamiltonian $H_{V}$. This means we have to foliate the same sphere diagram by intervals as in the right diagram of figure 3.1. These intervals are anchored at the two poles of the sphere, and it is natural to identify these two points with the two $\Omega$-points that appear in the sphere partition function. Treating the angular coordinate $\phi$ as a Euclidean time variable we will see that the sphere partition function $Z_{S^{2}}^{+}$naturally describes a canonical ensemble of open strings at finite temperature. We will further see that the modular Hamiltonian takes a similar form as in the closed string theory; it consists of a Nambu-Goto term plus local interactions that cut and reglue the open strings.

Note that at large $N$, the Gross-Taylor model contains two interacting chiral sectors. For simplicity, we will initially treat a single chiral sector, and generalize to the coupled theory in section 3.4. We will denote the partition function of a single chiral sector on the sphere as $Z_{S^{2}}^{+}$.

\subsubsection{Entanglement of the Hartle-Hawking state}

To set up our entanglement calculation we consider the Hartle-Hawking state $|H H\rangle$, which is prepared by the Euclidean path integral over the hemisphere geometry pictured in figure 3.8. This defines a state in the closed string Hilbert space associated with the circular boundary of the hemisphere. Wick rotated to Lorentzian signature, 


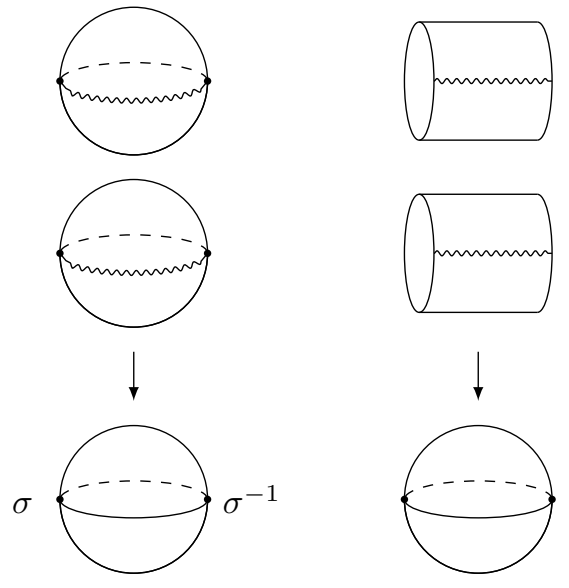

Figure 3.7: The figure on the left shows a term in the Gross-Taylor expansion corresponding to a two-sheeted branched covering of the sphere with two $\Omega$-point singularities. These two fixed points on the target sphere are labelled by the permutation $\sigma: 1 \rightarrow 2,2 \rightarrow 1$, since circling these points interchanges the two sheets. On the right, we have opened up each branch point on the covering space into a single connected boundary, representing an external closed string state given by $|\sigma\rangle$. In the open string channel, this is interpreted as a finite temperature loop diagram in which an open string anchored on the $\Omega$-points winds twice around the sphere. 
this state corresponds to the de Sitter-invariant vacuum state, which reduces to a thermal state in the static patch.

We will divide the circle into a semicircle $V \subset S^{1}$ and its complement $\bar{V}$. The entanglement between the degrees of freedom in $V$ and those in $\bar{V}$ is characterized by the reduced density matrix

$$
\rho_{V}=\operatorname{tr}_{\mathcal{H}_{\bar{V}}}|H H\rangle\langle H H|
$$

The unnormalized density matrix $\rho_{V}$ can be expressed as a path integral on the sphere with a cut along $V$ as in the right of figure 3.8. The sphere partition function $Z_{S^{2}}$ can be obtained by tracing over $V$, which corresponds to "sewing up" the cut in the sphere:

$$
Z_{S^{2}}=\operatorname{tr}_{\mathcal{H}_{V}} \rho_{V}=\operatorname{tr}_{\mathcal{H}_{V}} e^{-H_{V}}
$$

Given a suitable regularization of the entangling surface $\partial V$, we can regard $Z_{S^{2}}$ as a thermal partition function respect to the modular Hamiltonian $H_{V}:=-\log \rho_{V}$, which generates Euclidean time evolution in the angular direction orthogonal to $V$. The entanglement entropy is simply the thermal entropy of this ensemble at inverse temperature $\beta=1$.

Following the same steps that lead to the derivation of the Hamiltonian on the circle (8), one finds that the modular Hamiltonian $H_{V}$ is simply the quadratic Casimir operator $(50)$ acting on the Hilbert space $\mathcal{H}_{V}$. This is most easily proven when the size of the interval $V$ is exactly half the size of the great circle. In this case, the vector field $\xi^{a}$ that generates flow in entanglement time corresponds to a rigid rotation of the sphere, and the modular Hamiltonian of the Hartle-Hawking vacuum is the (suitably 
normalized) Killing energy which generates this symmetry [57]:

$$
H_{V}=2 \pi \int_{0}^{\pi} d \theta \sqrt{q} T_{a b} \xi^{a} n^{b}
$$

To compute $H_{V}$ explicitly we use spherical coordinates $d s^{2}=r^{2}\left(d \theta^{2}+\sin ^{2}(\theta) d \phi^{2}\right)$ and choose $V$ to be the line segment $\phi=0, \theta \in[0, \pi]$. The entangling surface then consists of the two poles $\theta=0$ and $\theta=\pi$. The Killing vector that fixes the entangling surface is $\xi=\frac{\partial}{\partial \phi}$ the unit normal is $n=\frac{1}{r \sin (\theta)} \frac{\partial}{\partial \phi}$ and $\sqrt{q}=r$ is the volume element on a slice of fixed $\phi$. The Yang-Mills energy density is $T_{\phi}^{\phi}=\frac{g_{\mathrm{YM}}^{2}}{2} \operatorname{tr}\left(E^{2}\right)=\frac{\lambda}{2 N} C_{2}$, which is constant over the interval, so we find:

$$
H_{V}=2 \pi \int_{0}^{\pi} d \theta r^{2} \sin (\theta) \frac{g_{\mathrm{YM}}^{2}}{2} \operatorname{tr}\left(E^{2}\right)=2 \pi r^{2} \frac{\lambda}{N} C_{2}=\frac{\lambda A}{2 N} C_{2}
$$

where we have used $A=4 \pi r^{2}$ for the sphere. Note that the modular Hamiltonian (37) is the same for an interval of any size on the sphere; by area-preserving diffeomorphism symmetry any two such intervals are equivalent, since the theory is only sensitive to the topology and total area.

The entanglement entropy is obtained by taking the derivative of the partition function

$$
S=\left.\left(1-\beta \partial_{\beta}\right) \log Z_{S^{2}}\right|_{\beta=1}
$$

This corresponds to a deformation of the background that introduces a small conical singularity at the entangling surface, taking the angular period from $2 \pi$ to $2 \pi \beta$. Since 2D Yang-Mills theory is only sensitive to the area and Euler characteristic $\chi$, this variation corresponds to varying the area linearly with $\beta$ while keeping $\chi$ fixed. Thus the entropy of the Hartle-Hawking state is given simply by:

$$
S=\left(1-A \partial_{A}\right) \log Z
$$


For more general situations (higher genus surfaces and larger numbers of intervals) the entropy involves analytically continuing the partition function in the Euler characteristic; we will not consider those cases here. ${ }^{7}$

Before giving the string description of the Hilbert space and modular Hamiltonian, we can describe them in terms of gauge theory variables. There the Hilbert space of an interval can be expressed in terms of states $|R, a, b\rangle$ where $R$ is an irreducible representation of $\mathrm{U}(N)$ and $a, b$ are indices in that representation. This basis diagonalizes the Casimir operator $C_{2}$, whose eigenvalues, which we denote we denote $C_{2}(R)$, depend only on the choice of representation. This leads to the expression for $Z_{S^{2}}$ in the representation basis:

$$
Z_{S^{2}}=\sum_{R}(\operatorname{dim} R)^{2} e^{-\frac{\lambda A}{2 N} C_{2}(R)}
$$

The additional factor of $(\operatorname{dim} R)^{2}$ comes from counting the edge modes of the gauge theory, which consist of one additional degree of freedom at each endpoint transforming in the representation $R$.

We will see that there is an analogous stringy interpretation of these edge modes. The Hilbert space of the interval $V$ can be described in terms of open strings. In the thermal open string interpretation of $Z\left(S^{2}\right)$, two infinitesimal disks containing the $\Omega$-points are removed, since the angular coordinate degenerates there. This results in a set of small disks cut out of the string worldsheet, each with a factor of $N$ associated with the sum over Chan-Paton indices. This is precisely what would be obtained by placing $N$ E-branes at a stretched codimension-1 entangling surface, and allowing open strings to end there. The edge modes in the string theory description are simply the Chan-Paton indices of the open strings.

\footnotetext{
${ }^{7}$ In these cases one cannot find a foliation of the manifold that degenerates at the entangling surface as in the case of a single interval on $S^{2}$. Thus we expect to find a nonlocal modular Hamiltonian.
} 


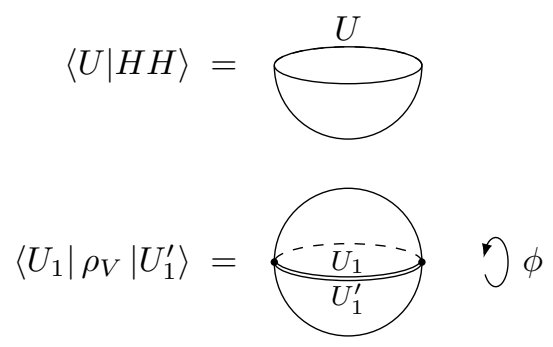

Figure 3.8:

The Yang-Mills path integral on a hemisphere gives the unnormalized Hartle-Hawking wave function, and the path integral on the sphere computes the square of its norm. Changing the periodicity of the angular coordinate $\phi$ to $2 \pi n$ yields $\operatorname{tr}\left(\rho_{V}^{n}\right)$.

We will also give an open string description of the modular Hamiltonian $H_{V}$ that will enable us to compute the thermal partition function (35) in the open string basis. In doing so, we will reproduce (32) and show that the closed string coupling $N=\frac{1}{g_{\text {string }}}$ to the $\Omega$-point in (32) arises from summing over $N$ Chan-Paton indices associated to open string endpoints anchored on the stretched entangling surface. To understand the origin of these edge modes we proceed by deriving the open string description of the Hilbert space on the interval $V$.

\subsubsection{The open string Hilbert space}

In order to describe entanglement of string states, we first need a stringy description of the Hilbert space of an interval. This Hilbert space is the space of squareintegrable functions on the group manifold, $L^{2}(G)$. Unlike the states on a circle, the states on an interval are not required to be class functions. Here we describe a class of open string states analogous to the closed string states of section 3.2.

Analogous to the closed string states of a circle, we introduce the following open string states of an interval. Consider a state of $n$ open strings, each carrying ChanPaton indices $i, j=1 \ldots N$. Letting $I=\left(i_{1}, \ldots, i_{n}\right)$ and $J=\left(j_{1}, \ldots, j_{n}\right)$, we define 


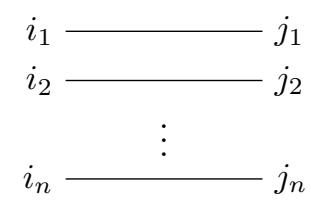

Figure 3.9:

The state $|I J\rangle$ represents a configuration of $n$ open strings with ChanPaton indices $\left(i_{1}, j_{1}\right) \ldots\left(i_{n}, j_{n}\right)$.

the state $|I, J\rangle$ by the wave functional

$$
\langle U \mid I, J\rangle=U_{i_{1} j_{1}} U_{i_{2} j_{2}} \cdots U_{i_{n} j_{n}}
$$

Note that the two Chan-Paton indices $i$ and $j$ of a string are distinguished, because one transforms in the fundamental representation, and the other transforms in the antifundamental. In other words, the open strings are oriented. We depict such an open string state in figure 3.9.

Note that unlike closed string states, open string states with different labels $I, J$ are not orthogonal. There are two independent reasons for this. The first is that open strings are indistinguishable, so $|I J\rangle$ and $|\sigma(I) \sigma(J)\rangle$ label the same state, when $\sigma$ is any permutation. This overcompleteness can be accounted for by labelling each such state with occupation numbers $\left\{n_{i j}\right\}_{i, j=1}^{n}$ which count the number of strings with Chan-Paton indices $(i, j)$. However there is a further non-orthogonality: the state $|I J\rangle$ has nontrivial overlap with $|I \sigma(J)\rangle$. This is a consequence of the nontrivial inner product on the space $L^{2}(G)$.

The Hilbert space of an open string is $L^{2}(\mathrm{U}(N), d U)$ where $d U$ is the Haar measure. We can use the matrix elements $U_{i j}$ as coordinates, and the Haar measure is given by:

$$
d U=\frac{\mathcal{N}}{\operatorname{det}(U)^{N}} \prod_{i, j} d U_{i j}
$$


We can check that this is invariant under left and right multiplication, which uniquely determines the Haar measure. ${ }^{8}$

The multiplication operator $U_{i j}$ can be viewed as a creation operator that creates an open string with Chan-Paton indices $(i, j)$. We can also consider the operator $\frac{\partial}{\partial U_{i j}}$ which annihilates an open string and satisfies $\left[\partial_{i j}, U_{k l}\right]=\delta_{i k} \delta_{j l}$. Unlike the usual ladder operators, the annihilation operator $\partial_{i j}$ is not the adjoint of the creation operator $U_{i j}$. To find its adjoint, we have to consider the inner product

$$
\begin{aligned}
\int d U f(U)^{*} \frac{\partial}{\partial U_{i j}} g(U) & =-\int \frac{\partial}{\partial U_{i j}}\left(\frac{\mathcal{N}}{\operatorname{det}(U)^{N}} f(U)^{*}\right) g(U) \prod_{k, l} d U_{k l} \\
& =\int d U\left(N U_{j i}^{-1} f(U)^{*}-\frac{\partial}{\partial U_{i j}} f(U)^{*}\right) g(U) \\
& =\int d U\left(N U_{i j} f(U)+U_{i k} U_{l j} \frac{\partial}{\partial U_{l k}} f(U)\right)^{*} g(U)
\end{aligned}
$$

In the last line we have used the unitarity condition, which implies

$$
\frac{\partial}{\partial U_{i j}}=-U_{k i}^{\dagger} U_{j l}^{\dagger} \frac{\partial}{\partial U_{k l}^{\dagger}}
$$

Thus we find that

$$
\left(\frac{\partial}{\partial U_{i j}}\right)^{\dagger}=U_{i k} \frac{\partial}{\partial U_{l k}} U_{l j}=N U_{i j}+U_{i k} U_{l j} \frac{\partial}{\partial U_{l k}}
$$

This suggests that to leading order in large $N$, we can treat the second term as subleading to the first, so that $U_{i j}$ and $\partial_{i j}^{\dagger}$ act as rescaled creation and annihilation operators. This is true as long as we consider states with a small number $n \ll N$ of strings, otherwise the second term receives an $n$-fold enhancement making its influence comparable to that of the first term. This approximation is discussed further in 3.3.6.

\footnotetext{
${ }^{8} \mathrm{Up}$ to the normalization factor $\mathcal{N}$ which plays no role in our discussion
} 


\subsubsection{Electric fields and quadratic Casimir}

Having described the Hilbert space of an interval in terms of open strings, we now give the open string description of the nonabelian electric field operators, and the quadratic Casimir which determines the modular Hamiltonian of an interval.

The Hilbert space of an interval carries two commuting actions of $\mathrm{U}(N)$, which are given by left and right multiplication. Their generators are the nonabelian electric fields at the endpoints of the interval, which we call the left and right electric fields.

The left electric field is given by

$$
E_{i j}^{L}=U_{i k} \frac{\partial}{\partial U_{j k}}
$$

This generates the left action of $\mathrm{U}(N)$ on itself when contracted with an $\mathfrak{u}(N)$ generator. The electric field satisfies $\left(E_{i j}^{L}\right)^{\dagger}=E_{j i}^{L}$, and its commutation relations represent the Lie algebra $\mathfrak{u}(N)$ :

$$
\left[E_{i j}^{L}, E_{k l}^{L}\right]=\delta_{j k} E_{i l}^{L}-\delta_{i l} E_{k j}^{L}
$$

Acting on the string states, the action of $E_{i j}$ is to transform strings with Chan-Paton indices $(j, k)$ into strings with Chan-Paton indices $(i, k)$. The diagonal element $E_{i i}^{L}$ (without summation on $i$ ) counts the number of open strings with left Chan-Paton index $i$.

There is also an analogous generator of transformations on the right,

$$
E_{i j}^{R}=U_{k i} \frac{\partial}{\partial U_{k j}}
$$

This satisfies the same algebra and adjoint relation as $E^{L}$. Moreover, the left and right electric fields commute:

$$
\left[E_{i j}^{L}, E_{k l}^{R}\right]=0
$$


Like in the closed string Hilbert space, we can define a quadratic Casimir operator $C_{2}$. Just as the Hamiltonian for evolution along a cylinder was proportional to $C_{2}$, the modular Hamiltonian for an interval on the sphere is also proportional to $C_{2}$. The Casimir is given in terms of the electric field as

$$
C_{2}=\operatorname{tr}\left(E^{2}\right)=\sum_{i, j} E_{i j}^{L} E_{j i}^{L}=\sum_{i, j} E_{i j}^{R} E_{j i}^{R}=N U_{i k} \frac{\partial}{\partial U_{i k}}+U_{i k} U_{j l} \frac{\partial}{\partial U_{j k}} \frac{\partial}{\partial U_{i l}}
$$

The Casimir operator commutes with both the left and right electric fields.

The Casimir operator (50) naturally splits into a leading term and an interaction subleading in the $1 / N$ expansion. The leading term just counts the number of open strings. The effect of the subleading quartic interaction is to cut two open strings, and glue them back together in a different order: we take two open strings with Chan-Paton indices $(j, k)$ and $(i, l)$ and replace them with strings with Chan-Paton indices $(i, k)$ and $(j, l)$. Note that the interaction preserves the number of strings, so the two terms commute. We write this as

$$
C_{2}=N n+2 H_{1}
$$

where the interaction term implements a transposition. The factor of 2 accounts for the double counting in the expression (51): for a fixed pair of strings the interaction term in (51) acts twice, once with the indices as $i j$ and once with them as $k l$. Introducing the permutation operators $\sigma$ such that

$$
\sigma|I J\rangle=|I \sigma(J)\rangle
$$

we can write $H_{1}$ as a sum over all transpositions:

$$
H_{1}=\sum_{\sigma \in T_{2}} \sigma
$$


Given a state $|\sigma\rangle$ in the closed string Hilbert space, we can view it as a state in the product of two open string Hilbert spaces as follows:

$$
|\sigma\rangle=\sum_{I J}|I J\rangle|J \sigma(I)\rangle
$$

While any closed string state can be written as a state in the tensor product of two open string Hilbert spaces, the converse is not true. This is because the states coming from closed strings have the further constraint that the number of states with left Chan-Paton index $i$ on one interval must equal the number of states with right Chan-Paton index $i$ on the other interval. We can see that this is a very significant restriction, as the closed string Hilbert space (at fixed $n$ ) has a dimension of order $N^{0}$, whereas the dimension of the open string Hilbert space grows as $N^{2 n}$. In the Yang-Mills description the restriction to closed string states corresponds to matching of the nonabelian electric field across the entanglement cut.

\subsubsection{The open string partition function}

Now we are ready to derive the Gross-Taylor expression (32) for the sphere partition $Z_{S^{2}}^{+}$in the open string channel. The open string partition function is simply the thermal partition function of the modular Hamiltonian:

$$
Z_{S_{2}}^{+}=\operatorname{tr}\left(e^{-\frac{\lambda A}{2 N} C_{2}}\right)
$$

where the trace is over the open string Hilbert space, and $C_{2}$ is the open string Casimir operator.

Now we can do a perturbative expansion of the interaction term $H_{1}$ to obtain open string Feynman diagrams. Since the interaction term commutes with the free 
term that counts the number of open strings, we can write

$$
\operatorname{tr}\left(\rho_{V}\right)=\sum_{n} e^{\frac{-n \lambda A}{2}} \sum_{i} \frac{(-1)^{i}}{i !}\left(\frac{\lambda A}{N}\right)^{i} \operatorname{tr}_{\mathcal{H}_{n}}\left(H_{1}^{i}\right)
$$

where here $\mathcal{H}_{n}$ is the sector of the Hilbert space with $n$ open strings. Due to open string indistinguishability, we must be careful to count each state only once. Let $T_{I J}$ denote the orbit of the state $I J, T_{I J}=\left\{\sigma(I) \sigma(J): \sigma \in S_{n}\right\}$. Since all elements of the orbit label equivalent open string states, we have to divide by the size of the orbit:

$$
\begin{aligned}
\operatorname{tr}\left(\rho_{V}\right) & =\sum_{n} e^{\frac{-n \lambda A}{2}} \sum_{i} \frac{(-1)^{i}}{i !}\left(\frac{\lambda A}{N}\right)^{i} \sum_{I J} \frac{1}{\left|T_{I J}\right|}\left\langle I J\left|H_{1}^{i}\right| I J\right\rangle \\
& =\sum_{n} e^{\frac{-n \lambda A}{2}} \sum_{i} \frac{(-1)^{i}}{i !}\left(\frac{\lambda A}{N}\right)^{i} \sum_{I J} \frac{1}{\left|T_{I J}\right|} \sum_{p_{1}, \ldots p_{i} \in T_{2}}\langle I J|p| I J\rangle .
\end{aligned}
$$

In the last line we have written the interaction term as a sum of transpositions $p_{1}, \ldots, p_{i}$ and denoted $p=p_{1} \cdots p_{i}$. The matrix element $\langle I J|p| I J\rangle$ is nonzero if and only if there is a permutation $\tau$ such that

$$
I=\tau(I), \quad p(J)=\tau(J)
$$

For a given $I, J$, we denote the stabilizer subgroup as $C_{I J}=\left\{\tau \in S_{n}: \tau(I)=\right.$ $I, \tau(J)=J\}$. The number of permutations satisfying (58) is the order of the stabilizer subgroup $\left|C_{I J}\right|$, since given any element $\tau$ satisfying the constraint, any other permutation $\sigma$ satisfying (58) must also belong to the coset $\tau C_{I J}$, which has the same number of elements as $C_{I J}$. Then using again the orbit-stabilizer theorem 
$n !=\left|T_{I J}\right|\left|C_{I J}\right|$, the sum over open string states at fixed $n, i$ gives:

$$
\begin{aligned}
\sum_{I, J} \frac{1}{\left|T_{I J}\right|}\langle I J \mid I p(J)\rangle & =\sum_{I J} \sum_{\sigma \in S_{n}} \frac{\delta(I, \sigma(I)) \delta(J, \sigma p(J))}{\left|T_{I J}\right|\left|C_{I J}\right|} \\
& =\frac{1}{n !} \sum_{\sigma \in S_{n}} N^{K_{\sigma}} N^{K_{p}-1_{\sigma}} .
\end{aligned}
$$

Each term in this sum counts the number of states compatible with an $n$-sheeted open string worldsheet on which interaction branch points $p_{1} \ldots p_{i}$ have been inserted in the bulk, opening branch cuts extending to the the boundary. As shown in figure 3.10, such branch cuts implement the exchange of Chan-Paton indices produced by the open string modular Hamiltonian. $K_{\sigma}$ and $K_{p^{-1} \sigma}$ are the number of distinct loops making up each of the worldsheet boundary and the open string partition function assigns $N$ states for each loop, corresponding to the $N$ E-branes on which the open strings can end. Our final result is:

$$
\operatorname{tr}\left(\rho_{V}\right)=\sum_{n} \frac{e^{\frac{-n \lambda A}{2}}}{n !} \sum_{i} \frac{(-1)^{i}}{i !}\left(\frac{\lambda A}{N}\right)^{i} \sum_{\sigma \in S_{n}} \sum_{p_{1} \ldots p_{i}} N^{K_{\sigma}} N^{K_{p^{-1}}}
$$

This reproduces the expected expression (32) for $Z_{S^{2}}^{+}$(up to a trivial relabelling $\left.p \rightarrow p^{-1}\right)$. But we have now seen how it arises as a trace over the open string Hilbert space: it is a thermal partition function describing the stringy entanglement thermodynamics of the Hartle- Hawking state. In particular, the thermal entropy of this open string ensemble (treating $A$ as an inverse temperature) coincides with the entanglement entropy of the interval $V$. Moreover, we see that the factors $N^{K_{\sigma}}$ and $N^{K_{p^{-1}}}$ have a statistical interpretation as counting the distinct Chan-Paton indices associated with the open strings. 
$\Sigma$
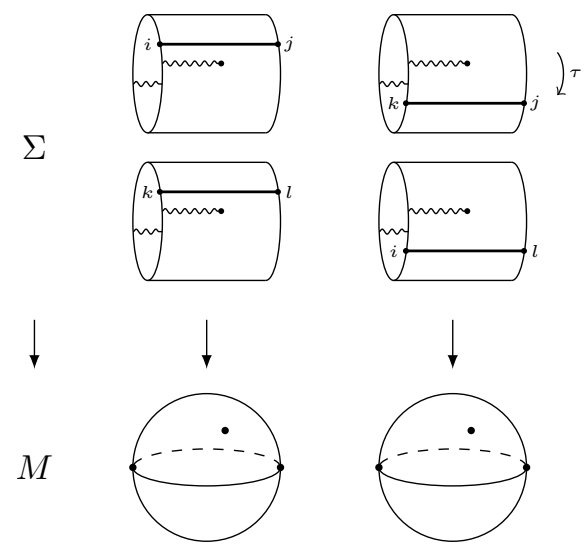

Figure 3.10:

Evolution under the open string modular Hamiltonian is represented as cylindrical worldsheets where time runs transverse to branch cuts. The figure shows a pair of open strings and their Chan-Paton indices at two different time slices. The indices on the left endpoints are exchanged as they pass through the branch cut. (The branch cut on the "back side" the cylinder connects both boundaries and does not correspond to open string interaction. This is the same branch cut that appears in figure 3.7.)

\subsubsection{The zero area limit}

To see more explicitly how the sphere partition function counts open string states, it is instructive to consider the zero area limit of $Z_{S^{2}}^{+}{ }^{9}$ Interpreted as a closed string amplitude, the zero area limit just gives the normalization $\langle\Omega \mid \Omega\rangle$. But in the open string channel, the zero area limit calculates the dimension of the open string Hilbert space, which counts the number of open string edge modes:

$$
\lim _{A>0} Z_{S^{2}}^{+}=\operatorname{tr}_{V}(\mathbf{1})=\sum_{n=1}^{\infty} \frac{1}{n !} \sum_{\sigma \in S_{n}} N^{2 K_{\sigma}}=\sum_{n=1}^{\infty} \frac{1}{n !} \sum_{k=1}^{n}\left[\begin{array}{l}
n \\
k
\end{array}\right] N^{2 k}=\sum_{n=1}^{\infty}\left(\begin{array}{c}
N^{2}+n-1 \\
n
\end{array}\right) .
$$

Here $\left[\begin{array}{l}n \\ k\end{array}\right]$ is the number of permutations in $S_{n}$ with $k$ cycles, also known as the (unsigned) Stirling number of the first kind. In the last line we have used the identity

\footnotetext{
${ }^{9}$ The zero area limit of the chiral Yang-Mills theory on any two-manifold gives a topological string theory that has been studied previously in ref. [19].
} 
that the generating function for $\left[\begin{array}{l}n \\ k\end{array}\right]$ is given by the "rising factorial"

$$
x^{(n)}:=\frac{(x+n-1) !}{(x-1) !}=\sum_{k=0}^{n}\left[\begin{array}{l}
n \\
k
\end{array}\right] x^{k} .
$$

The formula (62) appeared in [45], where it was calculated in the Yang-Mills theory by means of orthogonal polynomials. We now see that it has a natural interpretation in terms of the open string theory: it is the dimension of the open string Hilbert space, which counts the number of ways of assigning pairs of Chan-Paton indices $(i, j)=1 \ldots N$ to $n$ open strings, accounting for the indistinguishability of open strings. This is given by the number of weak compositions of $n$ into at most $N^{2}$ parts:

$$
\operatorname{dim} \mathcal{H}_{n}=\left[x^{n}\right](1-x)^{-N^{2}}=\left(\begin{array}{c}
N^{2}+n-1 \\
n
\end{array}\right)
$$

where $\left[x^{n}\right]$ is the operator that extracts the coefficient of $x^{n}$.

Finally, note that expressing $\lim _{A\rangle 0} Z_{S^{2}}^{+}$in the representation basis gives another formula for $\operatorname{dim} \mathcal{H}_{n}$ :

$$
\sum_{R \in Y_{n}}(\operatorname{dim} R)^{2}=\operatorname{dim} \mathcal{H}_{n}
$$

where $Y_{n}$ denotes the set of Young diagrams with $n$ boxes. This formula can be understood as follows. The symmetric group $S_{n}$ acts on $\mathcal{H}_{n}$ by permuting the right (or left) endpoints of the open string states $|I J\rangle$. The corresponding irreducible representations of $S_{n}$ are obtained by symmetrizing/antisymmetrizing the Chan-Paton indices according to the diagram $R \in Y_{n}$, and a basis for each such representation is given by the matrix elements $R_{a b}$ of the representation:

$$
\mathcal{H}_{n}^{R}=\bigoplus_{a, b}|R, a, b\rangle, \quad\langle U \mid R a, b\rangle:=R_{a b}(U)
$$


These representations are of dimension $(\operatorname{dim} R)^{2}$, so the left hand side of (65) merely counts the basis elements of $\mathcal{H}_{n}$ which block diagonalizes the action of $S_{n}$. This is the basis that diagonalizes the open string interaction Hamiltonian $H_{1}$, which also acts by permuting open string endpoints.

\subsubsection{The free chiral string}

Another approximation we can make to the chiral theory is to keep the area finite, but to neglect the interaction terms in the Hamiltonian (10). This theory can be studied at the level of the path integral by simply restricting the sum over worldsheets to those without interaction branch points; this calculation was carried out in ref. [84]. Generally when one truncates the path integral in some way there is no guarantee that the resulting expression continues to define a canonical partition function of the form tr $e^{-\beta H}$. Here we show that the partition function without branch points does define a canonical partition function of noninteracting open strings.

Unfortunately, the resulting partition function does not constitute a useful approximation to the interacting chiral partition function. As pointed out in ref. [45], the partition function has non-negligible contributions from states with $n \sim N^{2}$ strings. For these states there is an enhancement of the interaction term coming from the large number of strings which competes with the explicit factor of $1 / N$ and renders the interactions non-negligible. However, the free string remains an interesting illustrative example of the agreement between the sum over worldsheets and our canonical formulation.

The partition function of this theory is obtained by summing over maps of worldsheets into the target space with only one orientation and without interaction branch points. This sum was denoted $F_{O 1}$ in ref. [84]. The only allowed singularities are those at the $\Omega$-points, whose role is to count the open strings. The relevant connected diagrams are shown in figure 3.11. Each diagram consists of a cylinder wrapped $n$ times, 


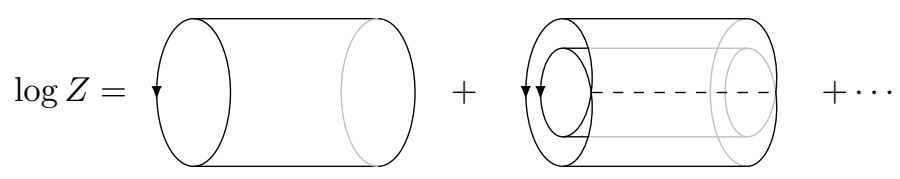

Figure 3.11:

In the free theory, the relevant diagrams are cylinders that wind around the direction corresponding to the entanglement time. The open ends of the cylinder are wrapped around the two $\Omega$-points.

whose weight in the path integral is given by the product of the Nambu-Goto action $e^{-\frac{\lambda A}{2} n}$, a symmetry factor of $1 / n$, and a factor of $N^{2}$ for the two open ends. This leads directly to the logarithm of the partition function:

$$
\log Z=N^{2} \sum_{n=1}^{\infty} \frac{e^{-\frac{\lambda A}{2} n}}{n}=-N^{2} \log \left(1-e^{-\frac{\lambda A}{2}}\right)
$$

In the canonical formulation of this theory, we drop the interaction term and the Hamiltonian simply counts the number of open strings weighted by the Nambu-Goto term. Since there are $N^{2}$ different labels for the open string endpoints, this leads to a partition function as sums over occupation $n_{i j}$ for the open string state with Chan-Paton indices $(i, j)$ :

$$
\log Z=\log \left(\prod_{i, j=1}^{N} \sum_{n_{i j}=0}^{\infty} e^{-\frac{\lambda A}{2} \sum_{i, j=1}^{N} n_{i j}}\right)=-N^{2} \log \left(1-e^{-\frac{\lambda A}{2}}\right)
$$

This is simply the logarithm of the partition function of $N^{2}$ harmonic oscillators, and agrees with the string path integral. Thus the replica trick applied to the sum over worldsheets without branch points computes the entropy of non-interacting open strings.

In the next section we consider the generalization to a theory with two chiral sectors. 


\subsection{The coupled theory}

So far we have focused on the stringy description of a single chiral sector of $2 \mathrm{D}$ Yang-Mills theory. Gross and Taylor showed that the full Yang-Mills partition function on $S^{2}$ is described by a closed string theory containing world sheets of two distinct orientations. In the sum over worldsheets one must account for a new type of singularity: orientation reversing tubes. These are additional singularities located only at the $\Omega$-points that connect string worldsheets of opposite orientation. Each such tube connects a chiral and antichiral string of the same winding number around the $\Omega$-point, and comes with a factor of $-1 / N^{2}$. Below we review the description of the closed string Hilbert space including both chiral sectors and show how the tube diagrams emerge from the left-right entanglement structure of the E-brane boundary state. We will then give an open string interpretation of the tubes and show that they arise from a counting of open string states taking into account the unitarity constraint $U U^{\dagger}=1$. Thus the orientation-reversing tube singularities arise as a natural feature of the open string kinematics.

\subsubsection{The coupled closed string Hilbert space}

As discussed briefly in section 3.2, our Hilbert space $\mathcal{H}^{+}$captures only one chiral sector of the Yang-Mills theory on a circle. In the representation basis, this sector contains representations whose Young diagrams have a fixed number of boxes as $N \rightarrow \infty$. In the string picture, these correspond to states obtained by acting on the vacuum with string creation operators $a_{k}^{\dagger}$ a finite number of times. The full Hilbert space also contains states whose energies $C_{2} \sim N$ are of the same order, but are not captured in this description because they correspond to representations whose Young diagrams have a number of boxes that scales with $N$. In particular, for any representation state $|S\rangle \in \mathcal{H}^{+}$one can consider the conjugate representation state $|\bar{S}\rangle \in \mathcal{H}^{-}$. Their closed string wave functions depend on $U^{\dagger}$ and satisfy a conjugate 
Frobenius relation (c.f. (9))

$$
\langle U \mid \bar{S}\rangle=\sum_{\tau \in S_{n}} \frac{\chi_{S}(\tau)}{n !}\langle U \mid \bar{\tau}\rangle,
$$

where the symmetrization rules for the Young diagrams are now applied to tensors of antifundamentals. The number of boxes in the Young diagram for $S$ does not scale with $N$, so the expression (69) has a well-defined limit as $N\rangle \infty$. The closed "antistring" states $|\bar{\tau}\rangle$ of this equation are defined as in (8), but with traces of powers of $U^{\dagger}$. These states belong to $\mathcal{H}^{-}$and can be visualized as collections of closed strings winding in the opposite direction around the spatial circle.

One can now construct a Hilbert space by combining the states $|\sigma\rangle$ and $|\bar{\tau}\rangle$. However, this is not a simple tensor product: $U$ and $U^{\dagger}$ are not independent due to the unitarity condition $U U^{\dagger}=1$. Treating the sectors as independent would therefore give an overcounting of the states. For example, the state with zero strings (corresponding to the trivial representation) will appear in every tensor product $R \otimes \bar{S}$ which contains the trivial representation.

We can avoid this overcounting by summing over only coupled representations. The coupled representation $R \bar{S}$ is defined as the largest irreducible representation that appears in the tensor product of representations $R \otimes \bar{S}$. Since these representations also have Young diagrams with $O(N)$ number of boxes, capturing their large $N$ limit requires a generalized version of the Frobenius relation. For $R$ and $S$ with $n$ and $\bar{n}$ boxes respectively, Gross and Taylor defined a set of coupled string states $|\sigma, \bar{\tau}\rangle$ such that

$$
\begin{aligned}
& |R \bar{S}\rangle=\sum_{\sigma, \tau} \frac{\chi_{R}(\sigma) \chi_{S}(\tau)}{n ! \bar{n} !}|\sigma, \bar{\tau}\rangle \\
& |\sigma, \bar{\tau}\rangle=\sum_{R, S} \chi_{R}(\sigma) \chi_{S}(\tau)|R \bar{S}\rangle
\end{aligned}
$$


where to leading order in $1 / N$ the coupled closed string state $|\sigma, \bar{\tau}\rangle$ has the wave function

$$
\langle U \mid \sigma, \bar{\tau}\rangle=\langle U \mid \sigma\rangle\langle U \mid \bar{\tau}\rangle+\cdots
$$

Substituting this leading approximation in (70) gives $|R \bar{S}\rangle=|R\rangle \otimes|\bar{S}\rangle$ so at leading order the coupled representation $R \bar{S}$ can be treated as a tensor product of $R$ and $\bar{S}$. At this order the closed string Hilbert space factorizes into a product of strings and antistrings:

$$
\lim _{N \rightarrow \infty} \mathcal{H}_{\mathrm{U}(N)}=\mathcal{H}^{+} \otimes \mathcal{H}^{-}
$$

The subleading corrections in (71) arise from subtracting the traces in the smaller irreducible representations that arise in $R \otimes \bar{S}$, creating entanglement between the two chiral sectors.

The general form of these correction terms can be deduced from the ClebschGordan rules for the Young diagrams, where the subleading terms are obtained from the leading one by "annihilating" strings and anti strings. The Clebsch-Gordan rules give the tensor product $R \otimes \bar{S}$ as a sum of representations whose diagrams are obtained from adding the boxes of $R$ to the boxes of $\bar{S}$. The largest of these representations is $R \bar{S}$, while the subleading ones correspond to different coupled representations $R^{\prime} \bar{S}^{\prime}$, in which $R^{\prime}$ and $S^{\prime}$ are obtained from $R$ and $S$ by deleting the same number $k$ of boxes from each. These representations correspond to states which are products of $n-k$ and $\bar{n}-k$ strings and antistrings. It is clear that the same coupled representation $R^{\prime} \bar{S}^{\prime}$ will occur in many different tensor products $R \otimes \bar{S}$, and the subleading terms in (71) correct for this overcounting. 
However, a simpler way to derive these correction is to impose orthogonality of the coupled closed strings basis [46]:

$$
\left\langle\sigma^{\prime}, \bar{\tau}^{\prime} \mid \sigma, \bar{\tau}\right\rangle=\delta_{T_{\sigma} T_{\sigma^{\prime}}} \delta_{T_{\tau} T_{\tau^{\prime}}}\left|C_{\sigma}\right|\left|C_{\tau}\right|
$$

where $T_{\sigma}$ denotes the orbit of the permutation $\sigma$, and the delta function ensures that $\sigma$ and $\sigma^{\prime}$ belong to the same orbit i.e. they are conjugate, and $C_{\sigma}$ counts the number of permutations commuting with $\sigma$. Equation (73) is necessary for the consistency of (70) with the orthonormality of the characters and imposing it leads to an exact expression for the coupled closed string basis [46]:

$$
|\sigma, \bar{\tau}\rangle=\sum_{\nu}(-1)^{K_{\nu}}\left|C_{\nu}\right||\sigma \backslash \nu\rangle|\bar{\tau} \backslash \nu\rangle
$$

Above, $\nu$ is a set of cycles of $\sigma$ for which there is a corresponding set of cycles of $\tau$ of the same lengths. The permutation $\sigma \backslash \nu$ is obtained by taking the cycles of $\sigma$ and deleting the set corresponding to $\nu . K_{\nu}$ is the number of cycles in $\nu$, which counts the number of strings and antistrings that have annihilated. Substituting (74) into (70) and choosing $U=U^{\dagger}=1$ immediately leads to the dimension formula.

$$
\operatorname{dim} R \bar{S}=\sum_{\sigma, \tau} \frac{\chi_{R}(\sigma) \chi_{S}(\tau)}{n ! \bar{n} !} \sum_{\nu}(-1)^{K_{\nu}}\left|C_{\nu}\right| N^{K_{\sigma \backslash \nu}} N^{K_{\bar{\tau} \backslash \nu}}
$$

Gross and Taylor used this formula to derive a large- $N$ expansion of the non-chiral partition function $Z_{S^{2}}$ and showed that that the subleading terms in $\frac{1}{N}$ such as $(75)$ could be expressed as string diagrams with orientation-reversing tubes.

\subsubsection{Entanglement tubes and the E-brane boundary state}

Here we will show that the tubes connecting oppositely oriented strings in the diagram expansion for $Z_{S^{2}}$ arise due to the left-right entanglement in the E-brane 
boundary state $|\Omega\rangle$. This is best illustrated in the zero area limit:

$$
\begin{aligned}
\lim _{A\rangle 0} Z_{S^{2}} & =\lim _{A\rangle 0}\left\langle\Omega\left|e^{-\frac{\lambda A}{2 N} C_{2}}\right| \Omega\right\rangle \\
|\Omega\rangle & =\sum_{R, \bar{S}} \operatorname{dim} R \bar{S}|R \bar{S}\rangle .
\end{aligned}
$$

As before, we have defined the E-brane boundary state $\langle U \mid \Omega\rangle$ as the Euclidean path integral on an infinitesimal disk. In the leading large- $N$ approximation where $\operatorname{dim} R \bar{S}=\operatorname{dim} R \operatorname{dim} S$, the boundary state factorizes:

$$
\begin{aligned}
|\Omega\rangle & \sim\left|\Omega^{+}\right\rangle\left|\Omega^{-}\right\rangle \\
\langle\sigma, \bar{\tau} \mid \Omega\rangle & \sim\left\langle\sigma \mid \Omega^{+}\right\rangle\left\langle\bar{\tau} \mid \Omega^{-}\right\rangle=N^{K_{\sigma}} N^{K_{\tau}} .
\end{aligned}
$$

The amplitude $\langle\Omega \mid \Omega\rangle$ also factorizes. Expressing $\left|\Omega^{ \pm}\right\rangle$in the coupled basis (74) leads to a diagrammatic expansion for $\langle\Omega \mid \Omega\rangle$ in which independent strings and antistrings propagate between $\Omega$-points living in the same sector. This is illustrated in the left figure in (3.12). As before, the coupling of the closed string states to the $\Omega$-point in each sector gives a factor of $N=\frac{1}{g_{\text {string }}}$ per closed string.

These diagrams must be corrected to account for the fact that the E-brane boundary state entangles the chiral and anti chiral sector. Using the dimension formula, we find that

$$
\begin{aligned}
|\Omega\rangle & =\sum_{R, \bar{S}} \operatorname{dim} R \bar{S}|R \bar{S}\rangle \\
& =\sum_{n, \bar{n}} \frac{1}{n ! \bar{n} !} \sum_{\substack{\sigma \in S_{n} \\
\tau \in S_{\bar{n}}}}\left(\sum_{\nu}(-1)^{K_{\nu}}\left|C_{\nu}\right| N^{K_{\sigma \backslash \nu}} N^{K_{\tau \backslash \nu}}\right)|\sigma, \bar{\tau}\rangle,
\end{aligned}
$$



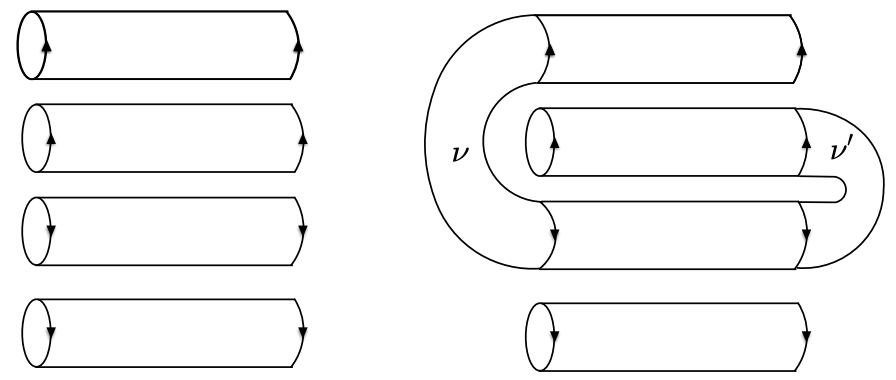

Figure 3.12:

Shown are two diagrams appearing in $\lim _{A\rangle 0}\left\langle\Omega\left|e^{-\frac{\lambda A}{2 N} C_{2}}\right| \Omega\right\rangle$. On the left,we have a decoupled closed string and anti string configuration. In the physical Hilbert space, strings and anti strings are entangled, leading to the diagram on the right where tubes $\nu$ and $\nu^{\prime}$ connect string-anti string pairs.

where $\nu$ is defined as before. The amplitude is corrected accordingly:

$$
\lim _{A\rangle 0}\left\langle\Omega\left|e^{-\frac{\lambda A}{2 N} C_{2}}\right| \Omega\right\rangle=\sum_{n, \bar{n}} \frac{1}{n ! \bar{n} !} \sum_{\substack{\sigma \in S_{n} \\ \tau \in S_{\bar{n}}}} \sum_{\nu, \nu^{\prime}}(-1)^{K_{\nu}}(-1)^{K_{\nu^{\prime}}}\left|C_{\nu}\right|\left|C_{\nu^{\prime}}\right| N^{K_{\sigma \backslash \nu}+K_{\tau \backslash \nu}} N^{K_{\sigma \backslash \nu^{\prime}}+K_{\tau \backslash \nu^{\prime}}}
$$

For a fixed $\sigma \in S_{n}, \tau \in S_{\bar{n}}$, the $\nu=0$ term describe the propagation of decoupled strings and anti -strings described by $\sigma$ and $\tau$, with the familiar coupling of $N=\frac{1}{g_{\text {string }}}$ per closed string (see left figure in (3.12)). For $\nu \neq 0$, each cycle of $\nu$ is represented by a tube that connects an external string antistring pair at one of the $\Omega$-points, causing them to annihilate. ${ }^{10}$ One such tube diagram is depicted in figure 3.12. Each tube decreases the number of holes in the worldsheet by 2, leading to a string coupling of $N^{-2}=g_{\text {string }}^{2}$. In addition, each of these annihilations comes with a factor of $(-1)$, and a factor of $\left|C_{\nu}\right|$ which reflects the way in which the tube changes the symmetry factor of the diagram.

\footnotetext{
${ }^{10}$ We can also think of the tube as part of a closed string worldsheet for a string that is emitted and absorbed by the same $\Omega$-point.
} 


\subsubsection{Open string description of the entanglement tubes}

To find a canonical open string interpretation of the entanglement tubes, we begin by considering the large- $N$ Hilbert space $\mathcal{H}$ on the interval $V$. In the representation basis, this Hilbert space is spanned by states $|R \bar{S}, a, b\rangle$ whose wavefunctions are matrix elements in the coupled representations:

$$
\langle U \mid R \bar{S}, a, b\rangle=[R \bar{S}]_{a b}(U)
$$

with $a, b=1, \ldots, \operatorname{dim}(R \bar{S})$ indices in the representation $R \bar{S}$. We are implicitly invoking a large- $N$ version of the Peter-Weyl theorem, which at finite $N$ says that the Hilbert space is spanned by matrix elements of the irreducible representations.

In the leading order of the $1 / N$ expansion where $R \bar{S}=R \otimes \bar{S}$, these matrix elements are obtained by symmetrizing/antisymmetrizing the open string states $|I J, K L\rangle$ which we define as

$$
\langle U \mid I J, K L\rangle=U_{i_{1} j_{1}} U_{i_{2} j_{2}} \cdots U_{i_{n} j_{n}} U_{k_{1} l_{1}}^{\dagger} U_{k_{2} l_{2}}^{\dagger} \cdots U_{k_{\bar{n}} l_{\bar{n}}}^{\dagger}
$$

These can be represented, as shown in figure 3.14, as a collection of $n$ open strings with Chan-Paton indices $\left(i_{1}, j_{1}\right), \ldots,\left(i_{n}, j_{n}\right)$ and $\bar{n}$ open antistrings with Chan-Paton indices $\left(k_{1}, l_{1}\right), \ldots,\left(k_{\bar{n}}, l_{\bar{n}}\right)$. The mixed symmetrization of Chan-Paton indices implements a projection onto the irreducible representations $R$ and $\bar{S}$ in each chiral sector, so summing over $R$ and $S$ removes this projection and results in a leading order Hilbert space $\mathcal{H}^{0}$ :

$$
\mathcal{H}^{0}=\bigoplus_{R, S, a, b}|R \otimes \bar{S}, a, b\rangle=\bigoplus_{I J, K L}|I J, K L\rangle
$$

where there are no symmetries imposed on the Chan-Paton indices. $\mathcal{H}^{0}$ is an extended Hilbert space in which $U$ and $U^{\dagger}$ are formally treated as independent matrices not 
constrained by the identity $U U^{\dagger}=1$. The subspace of physical states $\mathcal{H} \subset \mathcal{H}^{0}$ is obtained by projecting onto the irreducible representation $R \bar{S} \subset R \otimes \bar{S}$ in each term of the sum in (82). This projection is equivalent to enforcing the constraint $U U^{\dagger}=1$ on $\mathcal{H}^{0}$, thereby eliminating the linear dependence between the states $|I J, K L\rangle$ with different numbers of open strings.

To see why projection onto coupled representations is equivalent to the unitarity constraint, consider the space of matrix elements of $R \otimes \bar{S}$, where $R$ is an irreducible representation whose Young diagram has $n$ boxes, and $S$ is an irreducible representation whose Young diagram has $\bar{n}$ boxes. These states transform as $\mathrm{U}(N)$ tensors, so we may decompose them into irreducible representations of $\mathrm{U}(N)$ by standard methods. This simply amounts to subtracting out all possible traces which contract a fundamental index with an antifundamental index.

Let $\mathbf{P}$ denote the projection operator that projects each space $R \otimes \bar{S}$ to the coupled representation $R \bar{S}$. We can now see that the zero area limit of $Z_{S^{2}}$ is simply expressing a trace over the open string Hilbert space:

$$
\begin{aligned}
\lim _{A\rangle 0} Z_{S^{2}} & =\operatorname{tr}_{\mathcal{H}}(\mathbf{1}) \\
& =\operatorname{tr}_{\mathcal{H}}(\mathbf{P}) \\
& =\sum_{n, \bar{n}} \frac{1}{n ! \bar{n} !} \sum_{\substack{\sigma \in S_{n} \\
\tau \in S_{\bar{n}}}} \sum_{\nu, \nu^{\prime}}(-1)^{K_{\nu}}(-1)^{K_{\nu^{\prime}}}\left|C_{\nu}\right|\left|C_{\nu^{\prime}}\right| N^{K_{\sigma \backslash \nu}+K_{\tau \backslash \nu}} N^{K_{\sigma \backslash \nu^{\prime}}+K_{\tau \backslash \nu^{\prime}}} .
\end{aligned}
$$

This dimension formula can be understood by implementing the projection $\mathbf{P}$ systematically on the overcomplete set of states $|I J, K L\rangle$ in each sector of fixed $n$ and $\bar{n}$

The formula (83) is best illustrated by way of examples: let us first consider the case $n=\bar{n}=1$. To apply $\mathbf{P}$ to the state $|i j, k l\rangle$, we first subtract the tensor obtained 
from $U_{i j} U_{k l}^{\dagger}$ by contracting one pair of indices,

$$
U_{i j} U_{k l}^{\dagger} \rightarrow U_{i j} U_{k l}^{\dagger}-\frac{\delta_{j k}}{N} U_{i a} U_{a l}^{\dagger}
$$

We then do the same for the remaining two indices:

$$
\langle U|\mathbf{P}| i j, k l\rangle=\left(U_{i j} U_{k l}^{\dagger}-\frac{\delta_{j k}}{N} U_{i a} U_{a l}^{\dagger}\right)-\frac{\delta_{i l}}{N} U_{a j} U_{k a}^{\dagger}+\frac{\delta_{j k} \delta_{i l}}{N^{2}} U_{b a} U_{a b}^{\dagger}
$$

To see how this leads to the dimension formula (83), we need to count the number of independent states in (85), which amounts to enumerating the number of independent constraints we have imposed on the $N^{4}$ initial states due to the condition $U^{\dagger} U=1$. This can be done iteratively as follows. Start with the constraints involving one contraction:

$$
\begin{gathered}
U_{i a} U_{a l}^{\dagger}=\delta_{i l}, \\
U_{a j} U_{k a}^{\dagger}=\delta_{j k} .
\end{gathered}
$$

This gives a total of $2 N^{2}$ constraints. However these constraints are not independent because the constraint corresponding to contracting both indices simultaneously,

$$
U_{a b} U_{b a}^{\dagger}=N
$$

is counted in both (86) and (87). Thus the total number of independent states is

$$
\operatorname{dim} \mathcal{H}_{1,1}=N^{4}-N^{2}-N^{2}+1
$$

We can associate a diagram to each set of constraints in (86)-(88) with an open string for each $U$ and oppositely oriented antistring for each $U^{\dagger}$. Each contraction is represented by a line connecting a pair of open string endpoints as in figure (3.13). 


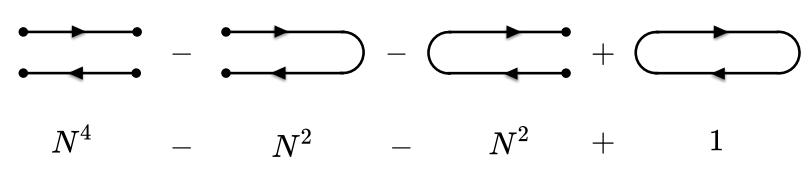

Figure 3.13: The left figure illustrates the diagrammatic computation for the number of open string edge modes for $n=\bar{n}=1$. As expected, this gives the square of the dimension of the adjoint representation, $\left(N^{2}-1\right)^{2}$.

Since these lines correspond to slices through the tube diagram in the closed string picture, we will also refer to them as tubes. Each tube reduces the number of free endpoints by 2 , and so decreases the number of states in the diagram by $N^{2}$. It also carries a factor of -1 arising from the fact that we are subtracting the trace. This counting is illustrated in figure 3.13, and indeed yields the correct number of states with $n=\bar{n}=1$.

When $n$ or $\bar{n}$ is greater than 1 , associating $N$ states per Chan-Paton index will overcount the number of states because of open string indistinguishability. More precisely, the string-antistring states are invariant under the action of the permutation group $S_{n} \times S_{\bar{n}}$ which relabels the strings within the same sector. Thus in performing the trace in (83) we should sum over all unrestricted Chan-Paton indices and divide by their orbit under this symmerty group. As in the chiral case, this will lead to a sum over diagrams in which Chan-Paton indices are correlated, and an overall symmetry factor of $n ! \bar{n} !$.

For example, the number of independent basis states contained $|I J, K L\rangle$ prior to the projection is:

$$
\begin{aligned}
\operatorname{dim} \mathcal{H}_{n, \bar{n}}^{0} & =\sum_{I, J, K, L} \frac{1}{\left|T_{I J, K L}\right|} \\
& =\sum_{I, J, K, L} \sum_{\sigma \in S_{n}} \sum_{\tau \in S_{\bar{n}}} \frac{\delta(I, \sigma(I)) \delta(J, \sigma(J)) \delta(K, \tau(K)) \delta(L, \tau(L))}{\left|T_{I J, K L}\right|\left|C_{I J, K L}\right|} \\
& =\frac{1}{n !} \frac{1}{\bar{n} !} \sum_{\sigma \in S_{n}} \sum_{\tau \in S_{\bar{n}}} N^{2 K_{\sigma}} N^{2 K_{\tau}}
\end{aligned}
$$


Here $T_{I J, K L}$ is the orbit of the open string state under $S_{n} \times S_{\bar{n}}$, and $C_{I J, K L}$ its stabilizer. This reproduces the leading term in (83), in which $\nu=\nu^{\prime}=\emptyset$.

The subleading terms in (83) accounts for indistinguishability at subsequent steps in the iterative counting of the constraints imposed by $\mathbf{P}$. For example, consider the constraints where a right index of an open string is contracted with a left index of an antistring:

$$
\sum_{a} U_{i_{1} a} \cdots U_{i_{n} j_{n}} U_{a l_{1}}^{\dagger} \cdots U_{k_{\bar{n}} l_{\bar{n}}}^{\dagger}=\delta_{i_{1} l_{1}} U_{i_{2} j_{2}} \cdots U_{i_{n} j_{n}} U_{k_{2} l_{2}}^{\dagger} \cdots U_{k_{\bar{n}} l_{\bar{n}}}^{\dagger} \cdot
$$

Just like the string states, the constraints can be labelled by multi-indices $(I, J)$ and $(K, L)$, except that $J$ and $K$ are allowed to contain one contracted index $a$. These indices are again invariant under the action of the symmetric group $S_{n} \times S_{\bar{n}}$, so we can write the number of non-identical constraints in (92) as

$$
\begin{aligned}
\sum_{I, J, K, L} \frac{1}{\left|T_{I J, K L}\right|} & =\sum_{I, J, K, L} \sum_{\sigma \in S_{n}} \sum_{\tau \in S_{\bar{n}}} \frac{\delta(I, \sigma(I)) \delta(J, \sigma(J)) \delta(K, \tau(K)) \delta(L, \tau(L))}{\left|T_{I J, K L}\right|\left|C_{I J, K L}\right|} \\
& =\frac{1}{n ! \bar{n} !} \sum_{\sigma \in S_{n}} \sum_{\tau \in S_{\bar{n}}}|\operatorname{Fix}(\sigma, \tau)| .
\end{aligned}
$$

The fix point set $\operatorname{Fix}(\sigma, \tau)$ consists of equations of the general form (92) that are invariant under $\sigma \times \tau$.

These are equations where the contracted index $a$ belongs to a 1-cycle in $\sigma$ and $\tau$ respectively. Meanwhile non-contracted indices must take the same value on each cycle of $\sigma$ and $\tau$, so there are $N^{2 K_{\sigma}-1} N^{2 K_{\tau}-1}$ elements in $\operatorname{Fix}(\sigma, \tau)$. This counting gives the terms in (83) in which $\nu$ is a cycle of length one and corresponds to all diagrams in which a single tube appears on the right side of the open strings. These diagrams are depicted in the middle in figure 3.14 .

At the next order in $1 / N$ we must account for the fact that the constraints themselves are not independent. The equations (92) are redundant because they satisfy re- 
lations obtained by contracting additional pairs of indices. For example, for $n=\bar{n}=2$ the $N^{6}$ constraints

$$
\sum_{a} U_{i_{1} a} U_{i_{2} j_{2}} U_{a l_{1}}^{\dagger} U_{k_{2} l_{2}}^{\dagger}=\delta_{i_{1} l_{1}} U_{i_{2} j_{2}} U_{k_{2} l_{2}}^{\dagger}
$$

are related by the $\frac{1}{2}\left(N^{4}+N^{2}\right)$ equations

$$
U_{i_{1} a} U_{a l_{1}}^{\dagger} U_{i_{2} b} U_{b l_{2}}^{\dagger}=\delta_{i_{1} l_{1}} \delta_{i_{2} l_{2}}
$$

However, to count the number of independent relations in (96), we must take care not to include the equations with $i_{1}=i_{2}$ and $l_{1}=l_{2}$, which take the form

$$
U_{i a} U_{a l}^{\dagger} U_{i b} U_{b l}^{\dagger}=\delta_{i l} \delta_{i l}
$$

This is just the square of a constraint on $\mathcal{H}_{1,1}^{0}$, so it should not be counted in $\mathcal{H}_{2,2}^{0}$. Excluding these $N^{2}$ equations, we conclude that the number of independent constraints in $(95)$ on the subspace $\mathcal{H}_{2,2}^{0}$ is

$$
N^{6}-\left(\frac{1}{2}\left(N^{4}+N^{2}\right)-N^{2}\right)
$$

These are represented by the $n=\bar{n}=2$ terms in (83) with nontrivial "tube" $\nu$ :

$$
\frac{1}{2 ! 2 !} \sum_{(\sigma, \tau) \in S_{2} \times S_{2}} \sum_{\nu \neq \emptyset}(-1)^{K_{\nu}}\left|C_{\nu}\right| N^{K_{\sigma}} N^{K_{\tau}} N^{K_{\sigma \backslash \nu}+K_{\tau \backslash \nu}}=-\frac{1}{4}\left(4 N^{6}-2 N^{4}+2 N^{2}\right)
$$

where the $N^{6}$ leading term comes from $\nu$ a single 1 -cycle, the $N^{4}$ correction corresponds to terms with $\nu$ a pair of 1-cycles and the $N^{2}$ term corresponds to $\nu$ being a 2-cycle. Comparing with (98) we see that the symmetry factor $C_{\nu}=2$ and the minus sign for the $\nu=(2)$ is exactly what's needed to exclude the $N^{2}$ redundant constraints in (97). 

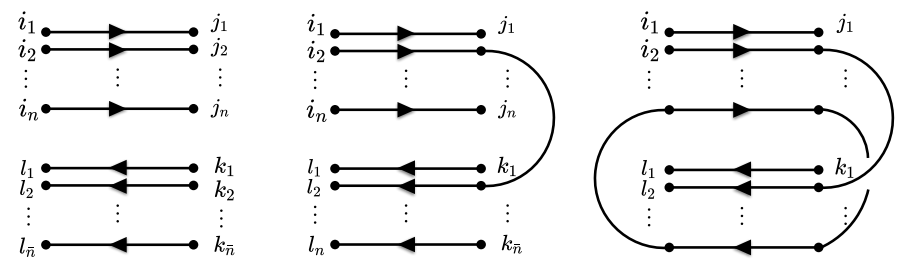

Figure 3.14:

On the left we have a diagram representing the states $U_{I J} U_{K L}^{\dagger}$. The diagram in the middle represents constraints imposed on $U_{I J} U_{K L}^{\dagger}$ obtained by contracting a pair of right endpoints. The right most figure shows another diagram with contractions on both the left and right endpoints

Including also the contractions of the other index, corresponding to terms with nontrivial $\nu^{\prime}$, gives the dimension of the space $\mathcal{H}_{2,2}$. Thus the alternating sign structure of (83) arises from the iterative counting of constraints imposed by the projection operator $\mathbf{P}$, and the zero area limit of the sphere partition enumerates the number of open string edge modes.

\subsubsection{Entanglement and modular Hamiltonian}

Having described the Hilbert space of the non-chiral string theory, all that remains to understand the entanglement entropy is the modular Hamiltonian. This is given by a multiple of the quadratic Casimir:

$$
H_{V}=\frac{\lambda A}{2 N} C_{2}
$$

where $C_{2}$ is defined as in (50). To see how this operator acts on the open string states, it is useful to lift it to the space $\mathcal{H}^{0}$ on which $U$ and $U^{\dagger}$ are independent. Applying 
the relation (44), we find that the Casimir can be written as a sum of chiral and antichiral pieces, plus a coupling term between the two sectors:

$$
\begin{aligned}
C_{2} & =C_{+}+C_{-}+C_{c}, \\
C_{+} & =N U_{i k} \frac{\partial}{\partial U_{i k}}+U_{i k} U_{j l} \frac{\partial}{\partial U_{j k}} \frac{\partial}{\partial U_{i l}}, \\
C_{c} & =-2\left(U_{i l} \frac{\partial}{\partial U_{i k}}\right)\left(U_{l j}^{\dagger} \frac{\partial}{\partial U_{k j}^{\dagger}}\right) .
\end{aligned}
$$

$C_{-}$has the same functional form as the $C_{+}$, but with $U \rightarrow U^{\dagger}$. Note that these operators are acting on $\mathcal{H}^{0}$, where the derivatives $\frac{\partial}{\partial U_{i j}}$ and $\frac{\partial}{\partial U_{i j}^{\dagger}}$ act independently. Here we see that the modular Hamiltonian contains all the terms expected from the worldsheet expansion. The leading term in $C_{ \pm}$leads to the Nambu-Goto area term, and the subleading term implements the branch point singularities that couple two open strings of the same chirality.

The coupling term $C_{c}$ generates an interaction between chiral and antichiral strings, and its presence ensures that $C_{2}$ commutes with the projector $\mathbf{P}$. One might wonder why there are no diagrams associated with the term $C_{c}$, which would correspond to local interactions coupling worldsheets of opposite orientations. The reason these diagrams do not appear is that the string states created by $C_{c}$ contain a trace $U_{i l} U_{l j}^{\dagger}$, and hence are annihilated by the projector $\mathbf{P}$.

The sphere partition function can be expressed as

$$
Z_{S^{2}}=\operatorname{tr}_{\mathcal{H}^{0}}\left(\mathbf{P} e^{-\frac{\lambda A}{2 N} C_{2}}\right)
$$

This completes our statistical accounting for the entanglement entropy of the HartleHawking state in the Gross-Taylor model. The entanglement entropy is given by the thermal entropy of a gas of non-chiral open strings with endpoints anchored on two stacks of E-branes located at the entangling surface, with interactions given by (101). 


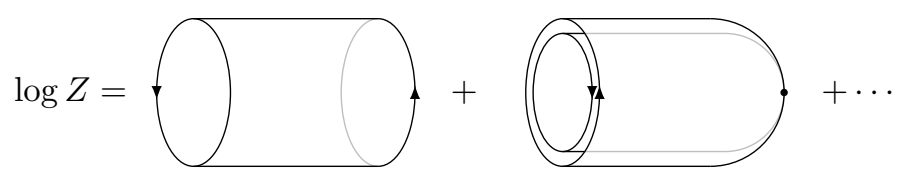

Figure 3.15:

The relevant connected diagrams for the free non-chiral string are cylinders that wind around entanglement time circle, and which collapse into an $\Omega$-point multiple times, each time re-emerging with the opposite orientation.

\subsubsection{The non-chiral free string}

As we did in section 3.3.6 for the chiral theory, we can consider the truncation of the coupled theory where interactions are neglected. This corresponds to a path integral over string worldsheets with two distinct orientations on a sphere with two $\Omega$-point singularities, and orientation-reversing tubes. This path integral was carried out in ref. [84] and was denoted $F_{O 2}$. In this case the relevant worldsheets consist of cylinders wrapping the entanglement time circle $n$ times. But now the cylinder can collapse into an $\Omega$-point in an orientation-reversing tube and emerge with the opposite orientation. A general connected diagram will cover the target space mn times, and have $m-1$ orientation-reversing tubes. The diagrams with $n=1$ and $m \geq 1$ are depicted in figure 3.15 . The resulting sum is naturally organized into a double sum over $m$ and $n$ :

$$
\begin{aligned}
\log Z & =2 N^{2} \sum_{m=1}^{\infty} \sum_{n=1}^{\infty} \frac{(-1)^{m-1}}{n} e^{-\frac{\lambda A}{2} m n} \\
& =2 N^{2} \sum_{m=1}^{\infty}(-1)^{m} \log \left(1-e^{-\frac{\lambda A}{2} m}\right)
\end{aligned}
$$

The terms with $m$ odd each give the logarithm of the partition function of a harmonic oscillator; but the terms with $m$ even give the partition function of a two-level system whose excited state comes with a negative Boltzmann weight. 
By manipulating the above expression, we can see that it takes the form of (the logarithm of) a canonical partition function with only positive norm states:

$$
\begin{aligned}
\log Z & =2 N^{2} \log \left(\sum_{n=0}^{\infty} e^{-\frac{\lambda A}{4} n(n+1)}\right) \\
& =2 N^{2} \log \left(\frac{\vartheta_{2}\left(0, e^{-\frac{\lambda A}{4}}\right)}{2 e^{-\frac{\lambda A}{16}}}\right)
\end{aligned}
$$

This describes $2 N^{2}$ uncoupled copies of a theory whose energy levels are the triangular numbers $T_{n}=n(n+1) / 2$. However, unlike the case of the chiral theory we do not have a description of the energy eigenstates that appear in the partition function in terms of string variables. Unlike the case of the chiral theory, they are not simply states with a definite number of open strings. We leave the canonical description of these states as an open puzzle.

However, we note that the expression in terms of the Jacobi $\vartheta$ function in (106) suggests a description in terms of bosonic free fields, and a connection to $R \rightarrow 1 / R$ duality as discussed in ref. [33]. We also note that the alternating signs in (104), which nevertheless cancel to yield a spectrum with only positive norm states, is reminiscent of the BRST-BV formalism. This formalism has been useful in formulating higherdimensional string field theory, see e.g. [74].

\subsection{Discussion and Future work}

We have shown how a description of entanglement between regions of space in the Gross-Taylor model necessitates a formulation of the theory in terms of open strings. We have shown that the entanglement entropy in the Gross-Taylor model counts open strings: more precisely the $\Omega$-point singularities discovered in ref. [46] count the number of distinct open string states accounting for both the indistinguishability of open strings, and the unitarity constraint. In the process, we have uncovered the 
E-brane; an object which sits at the entangling surface on which open strings can end.

Perhaps the most interesting product of our analysis is the relation between branes and entanglement. In a sense the effect of the E-brane is essentially just to change the statistical weight of certain configurations. However, the E-brane we have found acts in many respects like a D-brane, for example both are nonperturbative objects. It was shown in ref. [69] that the partition function in the presence of a D-brane state is of order $e^{-1 / g_{\text {string }}}$ and hence D-branes are nonperturbative objects. The $E$-branes have this same property: the partition function on the sphere satisfies $\log Z=O\left(N^{2}\right)$, and hence $Z \sim e^{-N^{2}}=e^{-1 / g_{\text {string }}^{2}}$ where we have identified $g_{\text {string }}=1 / N$. The square of the coupling reflects the fact that the sphere partition function has two E-branes. Hence E-branes are nonperturbative objects in the same sense that D-branes are.

An important question is to understand the dynamics of E-branes from the worldsheet perspective. One interesting clue from the sum over worldsheets is that the position of the E-branes is not integrated over. This is reminiscent of a D-brane, which is associated with Dirichlet boundary conditions that fix the location of the open string endpoints in spacetime. However our two-dimensional model is too simple to answer any more detailed dynamical questions. Due to the area-preserving diffeomorphism symmetry of the theory, the precise location of the E-brane is not a gauge-invariant concept. Moreover, the E-brane in this case has no transverse dimensions, leaving unanswered the question of how the transverse coordinates of open strings should be treated for higher-dimensional E-branes. Addressing these dynamical questions would necessitate studying a string theory with local degrees of freedom.

An example of a string theory with local degrees of freedom, closely related to the one considered here, is the critical bosonic string in two dimensions. This theory has a local degree of freedom described by a massless "tachyon" moving in a linear dilaton background. Recently the authors of [49] computed the spatial entanglement entropy 
of the tachyon by relating it to the collective field of a dual matrix model. Under this duality, the spatial entanglement of the tachyon is related to the entanglement in the space of eigenvalues of the large- $N$ matrix model. It is interesting to note that we can also formulate two-dimensional Yang-Mills theory as a (unitary) matrix model [66] with a collective field $\phi(\tilde{x})=\int d k a_{k} e^{i k \tilde{x}}$, where $\tilde{x}$ lives on a circle distinct from the one considered in our work. The Yang-Mills analog of the entanglement calculation in [49] is the entanglement entropy of the collective field in the $\tilde{x}$ coordinate.

Despite its apparent simplicity, there remain open questions about entanglement in the Gross-Taylor string theory. One such question is the description of entangling surfaces consisting of multiple intervals. The closed string formulation of twodimensional Yang-Mills theory holds on manifolds of higher genus as well, except that one must introduce $2 g-2$ " $\Omega^{-1}$-points". These $\Omega^{-1}$-points are analogous to the $\Omega$-points in that they allow for arbitrary singularities, but they are weighted differently in the path integral. We will not consider the higher genus case here, but it arises naturally in the calculation of entanglement entropy for multiple intervals. In that case, we do not expect to have a simple geometric description of the modular Hamiltonian as we do on the sphere, since the higher genus surfaces do not admit a foliation by intervals. Nevertheless, we expect to be able to describe the modular Hamiltonian as a combination of a geometric evolution, together with the insertion of topology-changing operators corresponding to the $\Omega^{-1}$-points.

An interesting phenomenon that appears on the sphere is the Douglas-Kazakov phase transition [34]. On the sphere there is a competition between the dimension term in the partition function which favors states with a large number of strings, and the quadratic Casimir which favors states with a small number of strings. Above the critical temperature, the string states no longer provide a good description. Given that we have identified that suitable Hilbert space of states in which the sphere partition function defines a canonical partition function, it would be of interest to 
find the states that provide a suitable weakly coupled description beyond the DouglasKazakov phase transition.

Another remaining open question is to find a $\sigma$-model Lagrangian whose partition function yields the sum over maps (see e.g. the discussion after eq (2.4) of ref. [45]). Hořava proposed such a string sigma model description in ref. [52]. It would be interesting to see if these $\sigma$ models could be generalized to open strings with endpoints anchored at the $\Omega$-points. This would help us to understand spacetime entanglement from the perspective of the string worldsheet, which will be important in treating higher dimensional string theories which don't have a simple string field theory description as in the case of the Gross-Taylor string.

A key question is whether this two-dimensional toy model can be used to gain insight into string theory entanglement in models with more spacetime dimensions. One possible way forward is via gauge-gravity duality: in the Hamiltonian formulation, lattice Yang-Mills theory is simply many copies of two-dimensional Yang-Mills theory, each defined on the links of the lattice, with couplings at the lattice sites. Thus at large $N$ we can describe lattice Yang-Mills theory as a theory of strings. This approach was pursued in ref. [63] to understand the emergence of a dual bulk. The open string description presented here may be useful in understanding the proposed duality for subregions [24].

Ultimately we would like to understand entanglement in higher-dimensional string theories with local degrees of freedom. One existing approach to this question is via gauge-gravity duality. In ref. [37] it was shown that subleading corrections to the Ryu-Takayanagi formula calculate bulk entanglement entropy via the replica trick. This derivation makes use of a brane-like surface extending into the bulk that acts as an entangling surface for the bulk field theory; it would be interesting to understand the coupling of strings to this surface, and whether it can be understood as counting states of the string endpoints. 
We have shown that the Gross-Taylor model provides a precise realization of Susskind's picture of entanglement entropy in string theory arising from genus-0 closed string diagrams. Here we see clearly that the leading order $N^{2}$ scaling of the entropy, which is necessary to obtain agreement with the $\sim 1 / G$ scaling of the Bekenstein-Hawking entropy, comes from the statistical weight of the Chan-Paton indices associated with open strings. This would seem to support the picture, suggested in refs. $[28,48]$ that the Bekenstein-Hawking term arises from bulk entanglement entropy, accounting for the appropriate (in this case stringy) edge modes. 


\section{Chapter IV}

\section{Entanglement edge modes in Chern Simons theory}

\subsection{Entanglement edge modes and topological phases}

In recent years, entanglement measures have become an important tool in condensed matter physics, where they are used as a diagnostic of many body states. Indeed, the scaling of entanglement entropy characterizes the amenability of systems to numerical simulations such as the density matrix renormalization algorithm (DMRG) in $1 \mathrm{~d}$, and the nature of the challenge in higher dimensions. Perhaps the most important application of entanglement arises in the study of gapped, topological phases. These phases are elusive because they cannot be detected by any local observables. One defining property of such phases is the appearance of gapless edge modes in the presence of a physical boundary. In [65], numerical evidence suggested that the spectrum of the modular Hamiltonian, the so-called entanglement spectrum, also contains these gapless modes and therefore provides a diagnostic for topological phases. Heuristically, this means that the entangling surface acts like a real boundary, and is therefore sensitive to the physical, gapless edge modes.

At low energies, universal features of topological phases can often be described by an emergent, topological gauge theory. In $2+1$ dimensions, Chern Simon theory provides the quintessential example of such a gauge theory, so it's natural to search for the connection between the entanglement spectrum and the physical edge modes 
here. In particular, the authors of [82] argued that the physical zero modes arise due to the presence of a boundary term in the modular Hamiltonian that should simulate the boundary term of the physical Hamiltonian. In this work we go a step further to identify the zero modes of the entanglement spectrum as the entanglement edge modes that are needed to achieve the Hilbert space factorization in Chern Simons theory. In the context of an IR emergent gauge theory the edge mode entanglement computed in the extended Hilbert space approach, which may at first glance seem to be gauge artifact, actually corresponds to physical entanglement in the underlying microscopic theory. In particular, the edge mode entanglement entropy in Chern Simons theory computes the topological entanglement entropy [? ], [89], which is an essential probe of topological phases.

Many of the results in this chapter have appeared in the literature in various guises. The appearance of physical edge modes in the entanglement spectrum of topological phases was first discussed in [65],[82],[70] and the computation of topological entanglement entropy in terms of left-right entanglement of boundary states was done in [22]. The reference [89], noted a problem with the approach of [70], where the reduced density matrix was obtained by a quantum quench in which region $A$ and $B$ are disconnected suddenly. The initial condition for such a quench is given by a conformally invariant boundary state $|B\rangle$ satisfying

$$
\left(L_{n}-\bar{L}_{-n}\right)|B\rangle=0
$$

where $L_{n}$ are the Virasoro generators. A general solution to this equation is given by a linear combination of Ishibashi states, which we will discuss in more detail below. Modular invariance dictates that $|B\rangle$ satisfies the Cardy condition, which implies that $|B\rangle$ is a particular linear combination of the Ishibashi states, with the coefficients given by elements of the modular S-matrix. Unfortunately, the state $|B\rangle$ 
does not reproduce the known entanglement entropies in Chern Simons theory, and the authors of [89] showed how to obtain the correct entropies by relaxing the Cardy condition.

In this chapter we offer a different perspective that justifies the methods of [89]. We carry out two simple derivations of the Chern Simons extended Hilbert space that lead to an explicit expression for embedding of the Chern Simons wavefunctionals into the extended Hilbert space. In section 4.2, we show how this embedding naturally arises from a careful UV regularization of the entangling surface in the path integral description of the reduced density matrix $\rho_{A}$. This section is essentially an application of the old arguments of Unruh [87] regarding the entanglement of the Minkowski vacuum to holographic TQFT's. In section 4.3 we arrive at the same results by implementing the "entangling product" defined in [28], which amounts to a gluing $H_{A}$ and $H_{B}$ in to the "bulk" Hilbert space $H$. In section 4 we discuss the calculation of entanglement entropy directly from the reduced density matrix. In the conclusion, we will end with some speculations about the description of the entanglement edge modes in the string theory dual to Chern Simons theory, which served as the original motivation for this work.

While this work was being competed, we became aware of [38] which takes a similar perspective on the edge modes of Chern Simons theory.

\subsection{Edge modes from the Euclidean Path integral}

\subsubsection{Path integral definition of the reduced density matrix}

In the ground state of a continuum QFT, the matrix elements of the (unnormalized) reduced density matrix $\tilde{\rho}_{A}$ can be represented by a path integral with a 
cut along region $A$

$$
\left\langle\phi_{A}\left|\tilde{\rho}_{A}\right| \phi_{A}^{\prime}\right\rangle=\int D[\phi] e^{-S[\phi]} \delta\left(\phi_{A^{+}}-\phi_{A}\right) \delta\left(\phi_{A^{-}}-\phi_{A}^{\prime}\right)
$$

where $A^{ \pm}$refers to the upper and lower edge of the cut. While this representation is adequate for the computing the entanglement entropy via the replica trick, it obscures the factorization of the Hilbert space. What should be done with the degrees of freedom living on the entangling surface $\partial A$, which naively belongs to both region $\mathrm{A}$ and B? As noted in recent works [15] [32], the resolution is to remove a small tubular neighborhood of the entangling surface, resulting in a stretched co-dimension 1 spacetime boundary. For certain backgrounds and choice of $A$ and $B$, we can choose the angular coordinate $\theta$ around the entangling surface as the Euclidean time coordinate. In such cases we can write the trace of $\tilde{\rho}_{A}$ as a path ordered exponential in $\theta$

$$
Z_{A}=\operatorname{tr}_{A} \tilde{\rho}_{A}=\mathrm{P} \exp \left(-\int_{0}^{2 \pi} K_{A}(\theta) d \theta\right)
$$

where $K_{A}$ generates translations in $\theta$. In a topological theory $K_{A}$ is a conserved charge independent of $\theta$ so this reduces to $Z_{A}=e^{-2 \pi K_{A}}$, which can be interpreted as a thermal partition function at temperature $2 \pi$. The Modular Hamiltonian $H_{A}=-\log \tilde{\rho}_{A}$ is thus a local Hamiltonian on $A$, and the entanglement entropy is identified with the thermal entropy of $Z_{A}$. Due to the presence of the stretched entangling surface, $H_{A}$ will in general have boundary terms describing the edge degrees of freedom. In the next section we will derive the Modular Hamiltonian for a disk $A$ in Chern Simons gauge theory and show how these edge modes are entangled.

\subsubsection{The extended Hilbert space of Chern Simons theory}

The Chern Simons Hilbert space on a closed, compact Riemann surface $\Sigma$ is equivalent to the space of conformal blocks of a chiral WZW model on $\Sigma$ [90]. On 
the other hand, when we make a spatial decomposition

$$
\Sigma=A \cup B
$$

the Chern Simons Hilbert spaces on $A$ and $B$ are those of the edge chiral WZW model on $\partial A$ and $\partial B$. By preparing states in $H_{\Sigma}$ with the Euclidean path integral we provide an explicit formula for its embedding into the extended Hilbert space

$$
H_{\Sigma} \subset H_{A} \otimes H_{B}
$$

where $H_{A}$ and $H_{B}$ are the chiral and anti chiral Hilbert spaces living entirely on the edge.

The simplest setting in which we can realize (5) explicitly is for the vacuum state on $\Sigma=S^{2}$ and a disk-like region $A$. This state $|\psi\rangle$ is prepared by the Euclidean path integral on a solid ball $B^{3}$ with the spatial $S^{2}$ as its boundary. Just as we did for the reduced density matrix, we will regulate this geometry by removing a semitubular neighborhood of the circle $\partial A$ of radial size $\epsilon$. We can then slice the path integral using an angular time coordinate $\theta$ that encircles the entangling surface. The advantage of this time slicing is that the (un-normalized) wave functional $\left\langle\phi_{A}, \phi_{B} \mid \psi\right\rangle$ can be viewed as an amplitude between states living on $A$ and $B$ [58]:

$$
\left\langle\phi_{A}, \phi_{B} \mid \psi\right\rangle=\left\langle\phi_{A}\left|e^{-\pi K_{A}} \mathcal{J}\right| \phi_{B}\right\rangle
$$

where $K_{A}$ generates rotations around the entangling surface and $\mathcal{J}$ is an anti-linear operator that implements a CPT transformation.

$$
\mathcal{J}: H_{B} \rightarrow H_{A}
$$




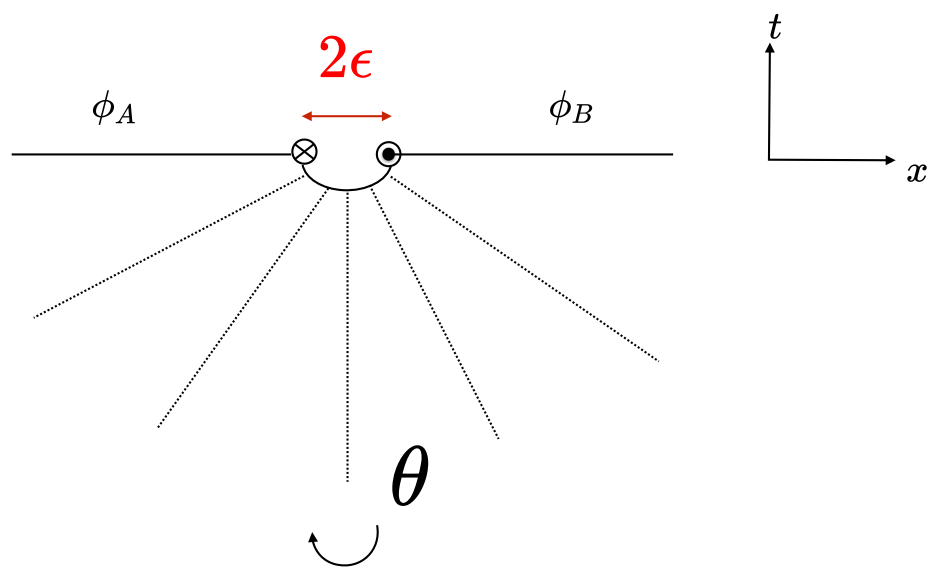

Figure 4.1:

The wavefunctional prepared by the Euclidean path integral can be sliced in angular time $\theta$, provided we remove an $\epsilon$ neighborhood of the entangling surface. When viewed as the initial and final time slices in $\theta$, region $A$ and $B$ are assigned opposite orientations relative to the ambient space $A \cup B$. Thus they support the Hilbert space of a chiral and anti-chiral edge CFT, which transforms non-trivially under the boundary gauge group. In addition to parity, switching from $t$ to $\theta$ also involves a time reversal because they have opposite orientations on region $B$ at the $t=0$ time slice. Sliced in angular time, the Euclidean path integral prepares a Thermofield double state in which the left right entanglement of these CFT's leads to singlet state under a diagonal action of the boundary gauge group on $\partial A$ and $\partial B$

The parity transformation is needed because the $\pi$ rotation on the RHS of (6) requires that $\left|\phi_{A}\right\rangle$ and $\mathcal{J}\left|\phi_{A}\right\rangle$ have opposite orientations, the $\mathrm{C}$ transformation accounts for the bra $\rightarrow$ ket mapping $\left\langle\phi_{A}|\rightarrow| \phi_{A}\right\rangle$, and the $T$ transformation accounts for the fact that $\theta$ and $t$ are oppositely oriented on region $B$ [56]. The operator $K_{A}$ evaluated at $\theta=0$ generates infinitesimal translations in the $t=x^{0}$ direction and is therefore the integral of the physical energy density $T_{00}$ weighted by a function $f(x)$

$$
K_{A}=\int_{A} d^{D-1} x f(x) T_{00}
$$


In the bulk of $A, T_{00}$ is zero. As noted earlier, quantizing Chern Simons theory on $A$ leads to a chiral CFT Hilbert space living on the edge. Thus the energy density is a delta function supported on the boundary $\partial A$, which we take to be the "stretched" surface at distance $\epsilon$ away from the entangling surface.

$$
T_{00}=\delta(\partial A) T_{\mathrm{CFT}} .
$$

In a neighborhood of the entangling surface we can choose local coordinates $\left(r, \theta, y^{i}\right)$, with $r$ a radial coordinate away from the entangling surface and $y^{i}$ parametrizing the surface itself. In this neighborhood, $f(x) \sim r$, so that $K_{A}$ takes the form of a (Euclidean) Rindler Hamiltonian. Due to the delta function in (9) we can relate $K_{A}$ to the Hamiltonian of the chiral edge CFT:

$$
K_{A}=\epsilon \int_{\partial A} T_{\mathrm{CFT}}=\epsilon H_{\mathrm{CFT}}
$$

For a circular boundary of length $l$ we can express the Hamiltonian in terms of the right moving Virasoro generator $L_{0}$ and the central charge $c$.

$$
H_{\mathrm{CFT}}=\frac{2 \pi}{l}\left(L_{0}-\frac{c}{24}\right)
$$

Expanding in a complete set of eigenstates of the Modular Hamiltonian in $A$ and $B$ in (6) and using the anti linearity of $\mathcal{J}$ leads to a representation of $|\Psi\rangle$ as a thermofield double state of the boundary CFT's.

$$
\begin{aligned}
\left\langle\phi_{A}\left|e^{-\pi K_{A}} \mathcal{J}\right| \phi_{B}\right\rangle & =\sum_{m, n}\left\langle\phi_{A} \mid n_{A}\right\rangle\left\langle n_{A}\right| e^{-\pi K_{A}} \mathcal{J}\left(\left|m_{B}\right\rangle\left\langle m_{B} \mid \phi_{B}\right\rangle\right) \\
& =\sum_{n, m}\left\langle\phi_{A} \mid n_{A}\right\rangle\left\langle n_{A}|\mathcal{J}| m_{B}\right\rangle\left\langle\phi_{B} \mid m_{B}\right\rangle e^{-\pi \epsilon E_{n}} \\
& =\left\langle\phi_{A} \phi_{B}\left|\sum_{m} e^{-\pi \epsilon E_{n}}\right| \bar{m}_{A}\right\rangle\left|m_{B}\right\rangle
\end{aligned}
$$


where we used the anti-linearity of $\mathcal{J}$ in the second line and denoted $\left|\bar{m}_{A}\right\rangle=\mathcal{J}\left|m_{B}\right\rangle$ in the third line [58]. Adding the normalization factor $Z_{A}$, we thus arrive at the extended hilbert space factorization of the "bulk" state $|\psi\rangle$

$$
|\psi\rangle=\frac{1}{Z_{A}} \sum_{n} e^{-\epsilon \pi E_{n}}\left|\bar{n}_{A}\right\rangle \otimes\left|n_{B}\right\rangle
$$

where $\left|n_{B}\right\rangle$ and $\left|\bar{n}_{A}\right\rangle$ are CPT conjugate states, corresponding to the right and left moving energy eigenstates of the edge CFT's, and $E_{n}$ are the right moving eigenvalues. Thus the bulk entanglement between the two disks are given entirely by the left-right entanglement of the edge CFT, as noted previously in [70], [22].

Equation (13) gives an explicit embedding of the state $|\psi\rangle$ into the extended Hilbert space. The state $|I\rangle=\sum_{n}\left|\bar{n}_{A}\right\rangle \otimes\left|n_{B}\right\rangle$ is an Ishibashi state [55], which solves the conformally invariant boundary condition

$$
\left(L_{m}-\bar{L}_{-m}\right)|I\rangle=0
$$

The thermo-field double state in (13) is a regularized Ishibashi state that has been rendered normalizable by evolving it with the CFT Hamiltonian for a small time $\epsilon$. We will see the relevance of (14) in the next section.

From (13) we can identify the $H_{\mathrm{CFT}}$ with the modular Hamiltonian. The reduced density matrix on $A$ is just the thermal density matrix at inverse temperature $2 \pi \epsilon$ :

$$
\rho_{A}=\frac{e^{-2 \pi \epsilon H_{\mathrm{CFT}}}}{Z_{A}}
$$

The normalization $Z_{A}$ is given by the finite temperature partition function of CFT.

$$
Z_{A}=\operatorname{tr} e^{-2 \pi \epsilon H_{\mathrm{CFT}}}
$$


Thus we see the edge modes partition function arise directly via the regularization of the entangling surface. For generic regions $A$ and $B$, we may not be able to find a globally well defined coordinate $\theta$ on the three manifold $M$ that evolves one region onto the other. ${ }^{1}$ In the next section we will provide another argument for the extended Hilbert space factorization, based on the requirements of gauge invariance in the presence of the entangling surface, which generalizes more readily to an arbitrary region $\mathrm{A}$.

\subsection{The entangling product for Chern Simons theory}

In the previous section we applied the extended Hilbert space construction [26], [30] in which a gauge invariant bulk state $|\psi\rangle \in H_{\Sigma}$ was factorized by embedding into a larger, non-gauge invariant Hilbert space $H_{A} \otimes H_{B}$ consisting of left and right moving edge modes. Now we consider this procedure in the opposite direction: starting with the Hilbert spaces $H_{A}$ and $H_{B}$, how do we glue them together to produce a gauge invariant subspace containing the bulk state $|\psi\rangle$ ? This "entangling product" was constructed for pure Yang Mills (and Einstein gravity) at the classical level and implemented quantum mechanically in the case of Yang Mills in 1+1 D in [28]. More recently, the classical phase space description of the entangling product was applied to abelian Chern Simons theory in [40],[38]. Below we will implement the quantum mechanical entangling product in Chern Simons theory.

\subsubsection{Hilbert space on a disk}

To begin, we recall how imposing gauge invariance in the presence of a boundary leads to new boundary degrees of freedom that transform under a boundary symmetry

\footnotetext{
${ }^{1}$ However, after regularizing, we can always find such a coordinate locally near the entangling surface that evolves $\partial A$ into $\partial B$. Since $T_{00}$ vanishes in the bulk, this may be sufficient to generalize the path integral argument to other regions.
} 
group. The Chern Simons action

$$
S=\frac{k}{4 \pi} \int_{\Sigma \times R} d^{3} x \operatorname{tr}\left(A \wedge d A+\frac{2}{3} A \wedge A \wedge A\right)
$$

is not gauge invariant in the presence of a boundary $\partial \Sigma$. One way to restore gauge invariance is to restrict gauge transformations to be trivial on the boundary. This means that the would-be pure gauge degrees of freedom living on $\partial \Sigma$ are promoted to dynamical degrees of freedom. These edge modes transform non trivially under the boundary gauge group, which is promoted to a physical symmetry.

To be concrete, we consider the quantization on a disk $\Sigma=D$, following the derivation in [35],[90]. First, we choose the boundary condition $A_{0}=0$, which ensures a well defined variational principle for the bulk equations of motion. The gauss law constraint $\frac{\delta S}{\delta A_{0}}=0$ restricts to flat connections on $D$, which are of the form.

$$
A=-\tilde{d} U U^{-1}
$$

where $\tilde{d}$ is the spatial exterior derivative on $D$, and $U \in G$ is an element of the gauge group $G$. Note that this is only pure gauge in the bulk, since gauge transformations are required to be trivial on the boundary. Inserting (18) back into the Chern Simons action gives the chiral WZW action

$$
S_{\mathrm{WZW}}=\frac{k}{4 \pi} \int_{\partial D \times R} \operatorname{tr}\left(U^{-1} \partial_{\phi} U U^{-1} \partial_{t} U\right) d \phi d t+\frac{k}{12 \pi} \int_{D \times R} \operatorname{tr}\left[\left(U^{-1} d U\right)^{3}\right]
$$

This action only depends on the boundary values ${ }^{2}$ of $U$. Gauge fixing and dividing by the volume of the gauge group in the bulk then leads to a boundary theory with

\footnotetext{
${ }^{2}$ The second term involves an integration over the bulk but for integer $k$ different bulk extensions of $U$ differ by integer multiples of $2 \pi i$, therefore giving the same exponential weighting in the path integral.
} 
the path integral

$$
Z_{\mathrm{bdry}}=\int d[U] e^{-S_{\mathrm{wZW}}}
$$

where $U: \partial D \times R \rightarrow G$. Thus Chern Simons theory on a disk is equivalent to a chiral WZW model living on the edge.

As alluded to earlier, the boundary symmetry group consist of the gauge transformation restricted to the boundary. In order to preserve the boundary condition $A_{0}=0$ these are also required to be time independent. Thus the boundary symmetry group is the loop group $L G$, whose elements

$$
g: S^{1} \rightarrow G,
$$

are maps from the boundary circle to the gauge group. Explicitly, the loop group elements acts on the WZW model via

$$
U \rightarrow g(\phi) U \text {. }
$$

The Hilbert space thus furnishes a representation of the loop group, or equivalently the current algebra of the gauge group G. The currents generating this algebra are just the boundary values of the gauge field

$$
\begin{array}{r}
J^{a}(z)=A_{z}^{a}=\left(\partial_{z} U^{-1} U\right)^{a} \\
a=1, . . \operatorname{dim} G
\end{array}
$$

where $z$ is a holomorphic coordinate on the boundary spacetime, and $a$ is a group index. 
For example, in the abelian case with gauge group $G=U(1), U=e^{i \theta}$ and the WZW model reduces to a chiral, compact boson $\theta$ with radius $\sim \frac{1}{k}$. The modes of the boson current $J(z)=\partial_{z} \theta$ satisfy the infinite dimensional current algebra at level $\mathrm{k}:$

$$
\left[J_{n}, J_{m}\right]=k n \delta_{n+m}
$$

Up to the $k$ dependent normalization, this is just an infinite set of harmonic oscillators, with the $n>0$ currents acting as annihilation and $n<0$ acting as creation operators. A representation of this algebra is obtained by applying $J_{-n}$ to the highest weight state $|0\rangle$ which is annihilated by $J_{n}$ for $n>0$. When $G$ is non-abelian, the level $k$ current algebra is

$$
\left[J_{m}^{a}, J_{n}^{b}\right]=i f^{a b c} J_{m+n}^{c}+k m \delta^{a b} \delta_{n+m}
$$

where $f^{a b c}$ are the structure constants of $G$. In this case the zero modes $J_{0}^{a}$ generate the finite dimensional Lie algebra of $G$ and the highest weight states $|r\rangle$ transforms in an irreducible representation $r$ of G. Only a subset of representations $r$ of $G$ admit the infinite dimensional generalization (25), and each corresponds to a primary field. For example, for $G=S U(2)$, the ground states transform in the usual $2 r+1$ dimensional representations with basis elements:

$$
|r, m\rangle,|m| \leq r
$$

and the Hilbert space is created from the highest weight state $|r, r\rangle$ via the ladder operators $J_{-n}^{a}$ and $J_{0}^{1}-i J_{0}^{2}$. The allowed "integrable" representations corresponds to half-integers $r$ satsifying $0 \leq r<k$. From the point of view of the bulk Chern Simons 
theory, states in different representations are prepared by a path integral Wilson line inserted inside $D \times R$ in the representation $r[90]$ [35].

\subsubsection{Gluing of Hilbert spaces}

Now let us return to the original question of how to glue together two Hilbert spaces $H_{A}$ and $H_{B}$. We take $A=D$ and $B=\bar{D}$ to be two oppositely oriented disks, which we wish to glue into a sphere $S^{2}=D \cup \bar{D} . H_{D}$ and $H_{\bar{D}}$ provides a representation of the chiral currents $J^{a}(z)$ anti-chiral currents $\bar{J}^{a}(\bar{z})$ respectively. Since the total Hilbert space $H_{S^{2}}$ is gauge invariant, whereas the tensor product $H_{D} \otimes \bar{H}_{\bar{D}}$ transforms non-trivially under $G$, we must restrict to a gauge invariant subspace. This subspace is denoted by the entangling product

$$
H_{S^{2}}=H_{D} \otimes_{G} H_{\bar{D}}
$$

defined as a quotient of the tensor product $H_{D} \otimes H_{\bar{D}}$ by the simultaneous action of the boundary symmetry group on $\partial D$ and $\partial \bar{D}$. In other words, a state $|\Psi\rangle \in$ $H_{A} \otimes_{G} H_{\bar{A}}$ is a singlet under the diagonal action of the loop group on the two edges of opposite chirality. Such a state is invariant under the current algebra and satisfies the constraint [55]

$$
\left(\mathbf{1}_{\mathbf{A}} \otimes J_{n}^{a}+\bar{J}_{-n}^{a} \otimes \mathbf{1}_{\mathbf{B}}\right)|\Psi\rangle=0
$$

For each representation of the current algebra corresponding to a primary with weight $r$, there is a solution to this equation given by the an Ishibashi state [55]

$$
|I, r\rangle=\sum_{n}|r, \bar{n}\rangle_{A} \otimes|r, n\rangle_{B}
$$


where $|r, n\rangle_{B}$ is a basis in the $r$ representation of the chiral current algebra, and the CPT conjugate $|r, \bar{n}\rangle_{A}=\mathcal{J}|r, n\rangle_{B}$ is the anti chiral counterpart. We can see that this is a solution by taking an arbitrary state $|\bar{a}\rangle|b\rangle$ and computing the the overlap

$$
\begin{aligned}
\left\langle\bar{a}\left|\otimes\left\langle b\left|\left(\bar{J}_{m}^{c}+J_{-m}^{c}\right) \sum_{n}\right| \bar{n}\right\rangle \otimes\right| n\right\rangle & =\sum_{n}\left\langle\bar{a}\left|\bar{J}_{m}^{c}\right| \bar{n}\right\rangle\langle b \mid n\rangle+\langle\bar{a} \mid \bar{n}\rangle\left\langle b\left|J_{-m}^{c}\right| n\right\rangle \\
& =\sum_{n}\langle b \mid n\rangle\left\langle a\left|\mathcal{J}^{\dagger} \bar{J}_{m}^{c} \mathcal{J}\right| n\right\rangle^{*}+\langle\mathcal{J} a \mid \mathcal{J} n\rangle\left\langle b\left|J_{-m}^{c}\right| n\right\rangle \\
& =\sum_{n}-\langle b \mid n\rangle\left\langle a\left|J_{m}^{c}\right| n\right\rangle^{*}+\left\langle b\left|J_{-m}^{c}\right| n\right\rangle\langle n \mid a\rangle \\
& =\sum_{n}-\langle b \mid n\rangle\left\langle n\left|J_{-m}^{c}\right| a\right\rangle+\left\langle b\left|J_{-m}^{c}\right| n\right\rangle\langle n \mid a\rangle=0
\end{aligned}
$$

Here we have made use of the identities $\langle\mathcal{J} a \mid \mathcal{J} b\rangle=\langle a \mid b\rangle^{*}$ and $\langle a \mid \mathcal{J} b\rangle=\left\langle\mathcal{J}^{\dagger} a \mid b\right\rangle^{*}$ due to the anti-linearity of $\mathcal{J}$ For the vacuum representation, normalizing the state (29) by applying the CFT evolution operator gives the same result obtained in the previous section by path integral methods. More generally, the choice of representation is determined by the bulk state $\left|\Psi_{r}\right\rangle$, which is prepared by the path integral on a solid ball $B^{3}$ with a Wilson line in the $r$ representation inserted. The endpoints of the Wilson line corresponding to anyonic charges $r$ and $\bar{r}$ are inserted inside $D$ and $\bar{D}$ of the sphere $S^{2}=\partial B$. Accounting for the normalization, such a state factorizes as

$$
\left|\Psi_{r}\right\rangle=\frac{1}{Z_{A}} \sum_{n} e^{-\pi \epsilon E_{n}}|r, \bar{n}\rangle_{A} \otimes|r, n\rangle_{B}
$$

The constraint (28), also implies the conformally invariant boundary condition (14). This is because the the generators of the current algebra is directly related to the Virasoro generators via the Sugawara construction [41]:

$$
L_{n} \sim \sum_{m}: J_{m+n}^{a} J_{-m}^{a}:
$$




\subsection{Entanglement entropy}

The entanglement entropy can be organized in a thermal form as a sum of a modular energy and a free energy term:

$$
S_{A}=-\operatorname{tr} \rho_{A} \log \rho A=\operatorname{tr}_{A}\left(\rho_{A} H_{A}\right)-\log Z_{A}
$$

When $A$ is a disk with charge $r$ inserted, we have

$$
S=2 \pi \epsilon \operatorname{tr}_{A}\left(\rho_{A} H_{\mathrm{CFT}}\right)+\log \left(\chi_{r}\left(e^{-\frac{2 \pi \epsilon}{l}}\right)\right)
$$

The first term vanishes as $\epsilon \rightarrow 0$, so the entropy comes entirely from the free energy, which we have written in terms of the Virasoro character $\chi_{r}$. The series is badly behaved as $\epsilon \rightarrow 0$ due to the infinite temperature limit, but can be computed by applying a modular transform [89].

$$
\chi_{r}\left(e^{-\frac{2 \pi \epsilon}{l}}\right)=\sum_{s} S_{r}^{S} \chi_{s}\left(e^{-\frac{2 \pi l}{\epsilon}}\right) \rightarrow S_{r 0} \chi_{0}\left(e^{-\frac{2 \pi l}{\epsilon}}\right)
$$

where in the last line we have retained the dominant term in the $\epsilon \rightarrow 0$ limit. In the limit, the partition function $\chi_{0}$ is dominated by the ground state with Casimir energy $E_{0}=\frac{-\pi c}{6}$, giving $\chi_{0} \sim e^{\frac{2 \pi l}{\epsilon} \frac{\pi c}{6}}$ This gives the entropy

$$
S_{A}=\frac{\pi^{2} c l}{3 \epsilon}+\log \left(S_{r 0}\right)
$$

The first term is the standard area law with explicit dependence on the regulator, and the second is the well known topological entanglement entropy. Since $S_{r 0}=\frac{d_{r}}{\mathcal{D}}$, where $d_{r}$ is the quantum dimension for the represention $r$ and $\mathcal{D}$ the total quantum 
dimension, we can write the topological term as

$$
S_{\mathrm{top}}=-\log \mathcal{D}+\log d_{r}
$$

We can interpret the extra term $\log d_{r}$ relative to the vacuum state as being due to the additional entanglement between anyonic charges. In the replica trick calculation, this arises because the path integral representation for $\operatorname{tr}_{A} \rho_{A}^{n}$ computes the expectation value of Wilson lines that have been inserted in the bulk [64].

\subsection{Conclusion}

In this work, we have provided two explicit derivations of the extended Hilbert space factorization of Chern Simons theory. Both are universal and applicable to holographic TQFT's which host edge modes in the presence of a boundary. The essential ingredient involved in both derivations is the regularization of the entangling surface, leading into a codimension one boundary. This is reminiscent of the stretch horizon in the study of black hole physics. The reduced density matrix describe a thermal ensemble of the edge theory and the entanglement entropy is given by the corresponding thermal entropy. The factorization of the bulk state into a maximally entangled Ishibashi state of two boundaries can interpreted as a gluing of two spacetimes along their edges. It would be interesting to apply this gluing in three dimensional gravity, which can also be formulated as a Chern Simons theory.

Chern Simons theory has no local degrees of freedom. Yet, the extended Hilbert space construction provides a precise definition of entanglement between spatial regions. In the presence of Wilson lines, we can think of the entanglement entropy ( relative to the vacuum with no insertions ) as being due to the cutting of the Wilson line by the entangling surface. String theory is another important example of a theory 
with non-local degrees of freedom. It is tempting to think that the fundamental string also induces entanglement across spatial regions when cut by an entangling surface.

Unfortunately, very little is known about the entanglement structure of string theory. It was conjectured long ago [80] that the entangling surface in string theory acts like a brane on which open strings end. Recently, we showed that this "entanglement brane" does indeed arise in a perturbative string calculation of entanglement entropy in a 2D string theory, dual to 2d Yang mills [32]. In that work, we showed that the entanglement brane provides a geometrical description of the entanglement edge modes in 2D Yang Mills.

Since Chern Simons theory is also dual to a (topological) string theory, it is natural to look for a string theory description of the entanglement edge modes in the form of a brane. Indeed this was the original motivation for this work. It has been known for sometime that the open string theory dual to $\mathrm{U}(\mathrm{N})$ Chern Simons theory on $S^{3}$ is the topological A-model on the deformed conifold $T^{*} S^{3}$, with $N$ branes wrapping $S^{3}$ [91] [42]. This open string theory is in turn dual to A model closed strings on the resolved conifold [42]. On this spacetime, the branes have been replaced by $N$ units of flux piercing the $S^{3}$. However, by analogy with our work in 2D Yang Mills, it is natural to ask whether branes will reappear in the closed string A model due to the presence of an entangling surface. We intend to pursue this question in the near future. 


\section{Bibliography}

[1] Davood Allahbakhshi, Mohsen Alishahiha, and Ali Naseh. Entanglement thermodynamics. arXiv preprint arXiv:1305.2728, 2013.

[2] John Baez and Washington Taylor. Strings and two-dimensional QCD for finite N. Nucl. Phys., B426:53-70, 1994.

[3] John C. Baez. Strings, loops, knots and gauge fields. In Knots and Quantum Gravity Riverside, California, May 14-16, 1993, 1993.

[4] Eric Benedict and So-Young Pi. Entanglement entropy of nontrivial states. arXiv preprint hep-th/9505121, 1995.

[5] Miguel Ibanez Berganza, Francisco Castilho Alcaraz, and German Sierra. Entanglement of excited states in critical spin chians. J.Stat.Mech., 1201:P01016, 2012.

[6] Jyotirmoy Bhattacharya, Masahiro Nozaki, Tadashi Takayanagi, and Tomonori Ugajin. Thermodynamical Property of Entanglement Entropy for Excited States. Phys. Rev. Lett. 110,, 091602, 2013.

[7] Jyotirmoy Bhattacharya and Tadashi Takayanagi. Entropic counterpart of perturbative einstein equation. arXiv preprint arXiv:1308.3792, 2013.

[8] Eugenio Bianchi. Horizon entanglement entropy and universality of the graviton coupling. preprint arXiv:1211.0522, 2012.

[9] Joseph J Bisognano and Eyvind H Wichmann. On the duality condition for a hermitian scalar field. Journal of Mathematical Physics, 16:985, 1975.

[10] Joseph J Bisognano and Eyvind H Wichmann. On the duality condition for quantum fields. Journal of mathematical physics, 17:303, 1976.

[11] L. Bombelli, R.K. Koul, J. Lee, and R.D. Sorkin. Quantum source of entropy for black holes. Physical Review D, 34(2):373-383, 1986.

[12] Luca Bombelli, Rabinder K. Koul, Joohan Lee, and Rafael D. Sorkin. Quantum source of entropy for black holes. Phys. Rev. D, 34(2):373-383, 1986.

[13] P. Calabrese and J. Cardy. Entanglement entropy and quantum field theory. J. Stat. Mech, 406(2), 2004. 
[14] P. Calabrese, J. Cardy, and E. Tonni. Entanglement entropy of two disjoint intervals in conformal field theory. J. Stat. Phys., 2009:P11001, 2009.

[15] John Cardy and Erik Tonni. Entanglement hamiltonians in two-dimensional conformal field theory. J. Stat. Mech., 1612(12):123103, 2016.

[16] John L Cardy. Conformal invariance and statistical mechanics. Les Houches, Session XLIX, pages 169-245, 1988.

[17] Horacio Casini, Marina Huerta, and Robert C. Myers. Towards a derivation of holographic entanglement entropy. JHEP, 1105:036, 2011.

[18] Stefan Cordes, Gregory W. Moore, and Sanjaye Ramgoolam. Lectures on 2D Yang-Mills theory, equivariant cohomology and topological field theories. Nucl. Phys. Proc. Suppl., 41:184-244, 1995.

[19] Stefan Cordes, Gregory W. Moore, and Sanjaye Ramgoolam. Large N 2D YangMills theory and topological string theory. Commun. Math. Phys., 185:543-619, 1997.

[20] Atish Dabholkar. Quantum corrections to black hole entropy in string theory. Phys. Lett., B347:222-229, 1995.

[21] Atish Dabholkar. Strings on a cone and black hole entropy. Nucl. Phys., B439:650-664, 1995.

[22] Diptarka Das and Shouvik Datta. Universal features of left-right entanglement entropy. Phys. Rev. Lett., 115(13):131602, 2015.

[23] Sebastian de Haro, Sergey N. Solodukhin, and Kostas Skenderis. Holographic reconstruction of space-time and renormalization in the ads / cft correspondence. Commun.Math.Phys., 217:595-622, 2001.

[24] Xi Dong, Daniel Harlow, and Aron C. Wall. Reconstruction of Bulk Operators within the Entanglement Wedge in Gauge-Gravity Duality. Phys. Rev. Lett., 117(2):021601, 2016.

[25] William Donnelly. Decomposition of entanglement entropy in lattice gauge theory. Phys.Rev., D85:085004, 2012.

[26] William Donnelly. Entanglement entropy and nonabelian gauge symmetry. Class. Quant. Grav., 31(21):214003, 2014.

[27] William Donnelly. Entanglement entropy and nonabelian gauge symmetry. Class. Quantum Grav., 31(21):214003, 2014.

[28] William Donnelly and Laurent Freidel. Local subsystems in gauge theory and gravity. 2016. 
[29] William Donnelly and Aron C. Wall. Do gauge fields really contribute negatively to black hole entropy? Phys.Rev., D86:064042, 2012.

[30] William Donnelly and Aron C. Wall. Entanglement entropy of electromagnetic edge modes. Phys. Rev. Lett., 114(11):111603, 2015.

[31] William Donnelly and Aron C. Wall. Geometric entropy and edge modes of the electromagnetic field. 2015.

[32] William Donnelly and Gabriel Wong. Entanglement branes in a two-dimensional string theory. 2016.

[33] Michael R. Douglas. Conformal field theory techniques in large $N$ Yang-Mills theory. In NATO Advanced Research Workshop on New Developments in String Theory, Conformal Models and Topological Field Theory Cargese, France, May 12-21, 1993, 1993.

[34] Michael R. Douglas and Vladimir A. Kazakov. Large N phase transition in continuum QCD in two-dimensions. Phys. Lett., B319:219-230, 1993.

[35] Shmuel Elitzur, Gregory W. Moore, Adam Schwimmer, and Nathan Seiberg. Remarks on the Canonical Quantization of the Chern-Simons-Witten Theory. Nucl. Phys., B326:108-134, 1989.

[36] Thomas Faulkner, Monica Guica, Thomas Hartman, Robert C. Myers, and Mark Van Raamsdonk. Gravitation from Entanglement in Holographic CFTs. JHEP, 03:051, 2014.

[37] Thomas Faulkner, Aitor Lewkowycz, and Juan Maldacena. Quantum corrections to holographic entanglement entropy. JHEP, 11:074, 2013.

[38] Jackson R. Fliss, Xueda Wen, Onkar Parrikar, Chang-Tse Hsieh, Bo Han, Taylor L. Hughes, and Robert G. Leigh. Interface Contributions to Topological Entanglement in Abelian Chern-Simons Theory. 2017.

[39] Dmitri V. Fursaev and Gennaro Miele. Cones, spins and heat kernels. Nucl.Phys., B484:697-723, 1997.

[40] Marc Geiller. Edge modes and corner ambiguities in 3d Chern-Simons theory and gravity. 2017.

[41] Paul H. Ginsparg. APPLIED CONFORMAL FIELD THEORY. In Les Houches Summer School in Theoretical Physics: Fields, Strings, Critical Phenomena Les Houches, France, June 28-August 5, 1988, pages 1-168, 1988.

[42] Rajesh Gopakumar and Cumrun Vafa. On the gauge theory / geometry correspondence. Adv. Theor. Math. Phys., 3:1415-1443, 1999. 
[43] Michael B Green, John H Schwarz, and Edward Witten. Superstring theory, vols. 1, 2. Cambridge, Uk: Univ. Pr.(1987)(Cambridge Monographs On Mathematical Physics), 1987.

[44] Andrey Gromov and Raul A. Santos. Entanglement entropy in 2D non-abelian pure gauge theory. Phys. Lett., B737:60-64, 2014.

[45] David J. Gross. Two-dimensional QCD as a string theory. Nucl. Phys., B400:161180, 1993.

[46] David J. Gross and Washington Taylor. Twists and Wilson loops in the string theory of two-dimensional QCD. Nucl. Phys., B403:395-452, 1993.

[47] David J. Gross and Washington Taylor. Two-dimensional QCD is a string theory. Nucl. Phys., B400:181-208, 1993.

[48] Daniel Harlow. The Ryu-Takayanagi Formula from Quantum Error Correction. 2016.

[49] Sean A. Hartnoll and Edward Mazenc. Entanglement entropy in two dimensional string theory. Phys. Rev. Lett., 115(12):121602, 2015.

[50] Song He, Tokiro Numasawa, Tadashi Takayanagi, and Kento Watanabe. Notes on entanglement entropy in string theory. JHEP, 05:106, 2015.

[51] Christoph Holzhey, Finn Larsen, and Frank Wilczek. Geometric and renormalized entropy in conformal field theory. Nucl.Phys., B424:443-467, 1994.

[52] Petr Hořava. Topological strings and QCD in two dimensions. In NATO Advanced Research Workshop on New Developments in String Theory, Conformal Models and Topological Field Theory Cargese, France, May 12-21, 1993, 1993.

[53] Veronika E Hubeny, Mukund Rangamani, and Tadashi Takayanagi. A covariant holographic entanglement entropy proposal. Journal of High Energy Physics, 2007(07):062, 2007.

[54] Ling-Yan Hung, Robert C Myers, Michael Smolkin, and Alexandre Yale. Holographic calculations of renyi entropy. Journal of High Energy Physics, 2011(12):1-61, 2011.

[55] Nobuyuki Ishibashi. The Boundary and Crosscap States in Conformal Field Theories. Mod. Phys. Lett., A4:251, 1989.

[56] T. Jacobson. Black hole thermodynamics and the space-time discontinuum. In Osgood Hill Conference: Conceptual Problems of Quantum Gravity Andover, Massachusetts, May 15-19, 1988, pages 597-599, 1988.

[57] Ted Jacobson. A Note on Hartle-Hawking vacua. Phys. Rev., D50:R6031-R6032, 1994. 
[58] Ted Jacobson. Black holes and Hawking radiation in spacetime and its analogues. Lect. Notes Phys., 870:1-29, 2013.

[59] Daniel N. Kabat. Black hole entropy and entropy of entanglement. Nucl. Phys., B453:281-299, 1995.

[60] Finn Larsen and Frank Wilczek. Geometric entropy, wave functionals, and fermions. Annals Phys., 243:280-298, 1995.

[61] Finn Larsen and Frank Wilczek. Renormalization of black hole entropy and of the gravitational coupling constant. Nucl.Phys., B458:249-266, 1996.

[62] Nima Lashkari, Michael B. McDermott, and Mark Van Raamsdonk. Gravitational dynamics from entanglement "thermodynamics". arXiv 1308.3716, 08 2013.

[63] Sung-Sik Lee. Holographic description of large $N$ gauge theory. Nucl. Phys., B851:143-160, 2011.

[64] Aitor Lewkowycz and Juan Maldacena. Exact results for the entanglement entropy and the energy radiated by a quark. JHEP, 05:025, 2014.

[65] Hui Li and F. Haldane. Entanglement Spectrum as a Generalization of Entanglement Entropy: Identification of Topological Order in Non-Abelian Fractional Quantum Hall Effect States. Phys. Rev. Lett., 101(1):010504, 2008.

[66] Joseph A. Minahan and Alexios P. Polychronakos. Equivalence of twodimensional QCD and the $C=1$ matrix model. Phys. Lett., B312:155-165, 1993.

[67] Masahiro Nozaki, Tokiro Numasawa, Andrea Prudenziati, and Tadashi Takayanagi. Dynamics of Entanglement Entropy from Einstein Equation. arXiv:1304.7100, 2013.

[68] Masahiro Nozaki, Tokiro Numasawa, and Tadashi Takayanagi. Holographic Local Quenches and Entanglement Density. arXiv: 1302.5703, 2013.

[69] Joseph Polchinski. Combinatorics of boundaries in string theory. Phys. Rev., D50:6041-6045, 1994.

[70] Xiao-Liang Qi, Hosho Katsura, and Andreas WW Ludwig. General relationship between the entanglement spectrum and the edge state spectrum of topological quantum states. Physical review letters, 108(19):196402, 2012.

[71] S. Ramgoolam. Comment on two-dimensional $O(N)$ and $S p(N)$ Yang-Mills theories as string theories. Nucl. Phys., B418:30-44, 1994.

[72] Shinsei Ryu and Tadashi Takayanagi. Aspects of holographic entanglement entropy. Journal of High Energy Physics, 2006(08):045, 2006. 
[73] Shinsei Ryu and Tadashi Takayanagi. Holographic derivation of entanglement entropy from ads/cft. Phys. Rev. Lett., 96:181602, 2006.

[74] Ashoke Sen. BV Master Action for Heterotic and Type II String Field Theories. JHEP, 02:087, 2016.

[75] Sergei N. Solodukhin. One loop renormalization of black hole entropy due to nonminimally coupled matter. Phys. Rev. D, 52:7046-7052, 1995.

[76] Rafael D. Sorkin. On the entropy of the vacuum outside a horizon. In Tenth International Conference on General Relativity and Gravitation (held Padova, 4-9 July, 1983), Contributed Papers, volume 2, pages 734-736, 1983.

[77] Mark Srednicki. Entropy and area. Phys. Rev. Lett., 71:666-669, 1993.

[78] Leonard Susskind. Some speculations about black hole entropy in string theory. 1993.

[79] Leonard Susskind and James Lindesay. Black holes, information and the string theory revolution. World Scientific, 2005.

[80] Leonard Susskind and John Uglum. Black hole entropy in canonical quantum gravity and superstring theory. Phys. Rev., D50:2700-2711, 1994.

[81] Brian Swingle. Structure of entanglement in regulated Lorentz invariant field theories. arXiv:1304.6402, 2013.

[82] Brian Swingle and T. Senthil. A Geometric proof of the equality between entanglement and edge spectra. Phys. Rev., B86:045117, 2012.

[83] Brian Swingle and Mark Van Raamsdonk. Universality of Gravity from Entanglement. 2014.

[84] Washington Taylor. Counting strings and phase transitions in 2D QCD. 1994.

[85] W. G. Unruh. Notes on black-hole evaporation. Phys. Rev. D, 14:870-892, Aug 1976.

[86] William G Unruh and Robert M Wald. What happens when an accelerating observer detects a rindler particle. Physical Review D, 29(6):1047-1056, 1984.

[87] William G. Unruh and Nathan Weiss. Acceleration Radiation in Interacting Field Theories. Phys. Rev., D29:1656, 1984.

[88] Mark Van Raamsdonk. Building up spacetime with quantum entanglement. Gen. Rel. Grav., 42:2323-2329, 2010. [Int. J. Mod. Phys.D19,2429(2010)].

[89] Xueda Wen, Shunji Matsuura, and Shinsei Ryu. Edge theory approach to topological entanglement entropy, mutual information and entanglement negativity in Chern-Simons theories. Phys. Rev., B93(24):245140, 2016. 
[90] Edward Witten. Quantum Field Theory and the Jones Polynomial. Commun. Math. Phys., 121:351-399, 1989.

[91] Edward Witten. Chern-Simons gauge theory as a string theory. Prog. Math., 133:637-678, 1995. 